\title{
SOME PROPERTIES OF THE FOURIER-TRANSFORM ON SEMISIMPLE LIE GROUPS. III
}

\author{
BY \\ L. EHRENPREIS AND F. I. MAUTNER( $\left.{ }^{1}\right)$
}

1. Introduction. This is a continuation of Parts I and II (see Ehrenpreis and Mautner [1] and [2]). Throughout the present Part III $G$ is still the group of conformal mappings of the unit disc. We continue our analysis and study of various classes of complex valued functions on $G$, using the methods and results developed in Parts I and II. In particular we are concerned with certain linear spaces of functions on $G$ which form (topological) algebras with convolutions as product, their explicit characterization by use of the Fourier transform on $G$, and their ideal theory. The Fourier transforms of the group algebra $D$ (of all indefinitely differentiable functions of compact support) and $E^{\prime}$ (the algebra of distributions of compact support) led to entire functions of exponential type. It is of considerable importance to describe explicitly algebras of functions on $G$ whose Fourier transform leads to functions which are regular in a given strip. Thus we shall introduce in $\$ 3$ below for each real number $p \geqq 1$ and $\leqq 2$ an algebra $S^{p}$ of functions $f(g)$ on $G$ whose Fourier transform we can again describe completely. Using this we can study the ideals of $S^{p}$. For $p=2$ we obtain the rather complete result that every closed two-sided ideal of $S^{2}$ is the intersection of the primary two-sided closed ideals containing it. The results of $\$ 3$ are used in $\$ 4$ to obtain further results about the expansion of mean-periodic functions on $G$ obtained in $\$ 5$ and $\$ 6$ of Part II. To study the spectrum of a two-sided mean-periodic function on $G$ in detail we consider (in $\$ 5$ below) the closed two-sided ideals of the groupalgebras $D$ and $E^{\prime}$. One of the main results of this section is a rather complete description of the closed two-sided ideals of $D$ and $E^{\prime}$ in terms of a spectrum which consists of a (discrete) subset of the complex plane with certain integers (orders of vanishing) attached to its points. This can be used (in §6) to introduce the spectrum of any two-sided mean periodic function on $G$ and to relate it to the expansion of $\S 5$ and $\S 6$ of Part II. In the next section ( $\$ 7)$ we study ideals of $L^{1}(G)$ and related algebras; among other things we show that an analogue of Wiener's theorem is false. We thus obtain a rather comprehensive study of various function spaces on $G$.

Functions on homogeneous spaces of a noncompact group behave in gen-

Received by the editors May 8, 1957.

(1) Except for minor modifications and additions this part III was written during the academic year 1955-1956 at the Institute for Advanced Study and was partially supported by the National Science Foundation and the Office of Ordnance Research. During that time the second named author was a Fellow of the John Simon Guggenheim Memorial Foundation. 
eral in a quite different manner from functions on the group itself and their study requires new methods. In $\S 8$ we treat the case where $\Gamma$ is any discrete subgroup of $G$ for which the factor space $G / \Gamma$ is compact. We study the Fourier expansion on $G / \Gamma$. This leads to an analogue of the Frobenius reciprocity theorem, also to a new analogue of the Poisson-summation formula. In $\S 9$ we drop the assumption that $G / \Gamma$ be compact, but restrict ourselves to the case where $\Gamma$ is the modular group. We obtain an explicit characterization of a class of modular functions in terms of analytic functions satisfying the functional equation of the zeta function of the field of Gaussian numbers.

We take this opportunity to correct some misprints in Part II :

p. 30, line -7: $\quad P_{m n} W \cdot f=\int \bar{w}_{m n}(g) f(g) d g$, where $w_{m n}=P_{m n} w$,

p. 31, line 2: $\quad \bar{W}$ is defined by: $\bar{W} \cdot h=$ complex conjugate of $W \cdot \bar{h}$.

p. 42, line -3: (4.32) $\int \bar{h}(g) u_{m n}\left(g, \frac{1}{2}+i t\right) d g=0$,

p. 43 , line 1: $\quad(4.33) \int \bar{f}(g) u_{m n}\left(g, \frac{1}{2}+i t\right) d g=0$.

p. 46 , lines -2 and -1 : $\quad W \cdot a * f=a^{*} \cdot W * f=a^{*} \cdot f * W^{*}$ where $a^{*}(g)=\bar{a}\left(g^{-1}\right)$.

Throughout the rest of $\S 5(\check{a})^{*}$ should be replaced by $a^{*}$ and $(\breve{W})^{*}$ by $W^{*}$.

2. The Fourier transform of $L^{p}$. Let $L^{p}=L^{p}(G)$ denote the Lebesgue space of all (equivalence classes of) complex-valued measurable functions $f(g)$ on $G$ for which

$$
\|f\|_{p}=\left\{\int_{G}|f(g)|^{p} d g\right\}^{1 / p}<\infty
$$

where $d g$ refers to Haar-measure on $G$ (normalized once and for all). By $L_{m n}^{p}$ we denote the subspace of all those $f(g) \in L^{p}$ which are spherical of type $m, n$ (the space $L_{\infty}^{1}$ was denoted by $A_{1}$ in Part I). Note that $f \in L_{m n}^{p}$ is equivalent to

$$
\int_{0}^{\infty}\left|f\left(g_{\zeta}\right)\right|^{p} \sinh 2 \zeta d \zeta
$$

being absolutely convergent (in the sense of Lebesgue) because $\left|f\left(k g_{\xi} k\right)\right|$ $=\left|f\left(g_{5}\right)\right|$ for $f(g)$ spherical.

Let us consider the function $h(g)$ on $G$ defined by $h\left(k g_{\xi} \kappa\right)=e^{-2 c \xi} f\left(k g_{\xi} \kappa\right)$.

Lемма 2.1. If $f(g) \in L_{m n}^{p}$ then $h(g) \in L_{m n}^{p}$ for any real number $c>1 / q$ where

$$
\frac{1}{p}+\frac{1}{q}=1 \text {. }
$$

Moreover the integral $\int_{0}^{\infty} e^{-2 c \xi} f\left(g_{\xi}\right) \delta_{\xi}$ converges absolutely and uniformly in $c$ for $c \geqq 1 / q+\epsilon,(\epsilon>0)$.

Proof. To prove that $h(g) \in L_{m n}^{1}$ it is sufficient to prove that $e^{-2 c \zeta} \in L^{q}$ $(0<\zeta<\infty ; \delta \zeta)$ because $\left|f\left(k g_{\zeta} \kappa\right)\right|=\left|f\left(g_{\zeta}\right)\right|$ for $f(g)$ spherical. Now 


$$
\begin{aligned}
\int_{0}^{\infty}\left|e^{-2 c \zeta \mid}\right| \alpha \delta \zeta & =\int_{0}^{\infty} e^{-2 c \zeta q} \sinh 2 \zeta d \zeta \\
& <\int_{0}^{\infty} e^{-2(1 / q+\epsilon) \zeta q} \sinh 2 \zeta d \zeta<\int_{0}^{\infty} e^{-2 q \epsilon \zeta} d \zeta
\end{aligned}
$$

for $c>1 / q+\epsilon$. The last integral exists and is independent of $c$, which proves Lemma 2.1 .

Lemma 2.2. Let $s$ be any complex number in the strip $0<R s<1$. Then

$$
\left|u_{m n}\left(g_{\xi}, s\right)\right| \leqq \text { const. } e^{-2 \xi\{\min (\sigma, 1-\sigma)\}}
$$

where the constant is independent of $m, n$ and $\xi$. If $s$ is in a strip $\epsilon<R s<1-\epsilon$ $(\epsilon>0)$ then the constant is also independent of $s$.

Proof. Consider first $u_{00}(g, s)=\phi(g, s)$. In accordance with (4.9) of Part I (p. 4.20) there are functions $\phi^{(1)}(g, s)$ and $\phi^{(2)}(g, s)$ such that $\phi(g, s)=\phi^{(1)}(g, s)$ $+\phi^{(2)}(g, s)$, where $\phi^{(2)}(g, s)=\phi^{(1)}(g, 1-s)$ and $\phi^{(1)}\left(g_{5}, s\right)=O\left(e^{-25 \sigma}\right)$ uniformly in $s$ for $R s=\sigma>\epsilon>0$ and all $\xi>0$. This proves Lemma 2.2 for $(m, n)=(0,0)$. The general case now follows from the inequality $\left|u_{m n}(g, s)\right| \leqq \phi(g, \sigma)$ (see e.g. p. 412 of Part I).

THeOREM 2.1. If $f(g) \in L^{p}$ then

$$
F_{m n}(s)=\int_{G} f(g) u_{m n}(g, s) d g
$$

converges for fixed $m, n$ and $s$ absolutely (Lebesgue) provided

$$
1 / q<R s<1 / p
$$

and uniformly in $m$ and $n$, and $s$ for

$$
1 / q+\epsilon \leqq R s \leqq 1 / p-\epsilon, \quad(\epsilon>0) .
$$

Hence $F_{m n}(s)$ is a regular analytic function of $s$ in the open strip (2.4).

Theorem 2.1 is an immediate consequence of Lemmas 2.1 and 2.2.

3. The group algebra $S^{p}$, its Fourier transform and ideal theory. For any real number $p \geqq 1$ and $\leqq 2$ let $S_{m n}^{p}$ consist of all indefinitely differentiable spherical functions $f(g)$ on $G$ which are of fixed type $m, n$ and satisfy

$$
(\partial f)\left(k g_{\zeta} \kappa\right)=O\left(\frac{e^{-2 \zeta / p}}{1+|\zeta|^{j}}\right)
$$

for all integers $j \geqq 0$ and for every polynomial $\partial$ in the elements of the Lie algebra of $G$ (considered as both left and right differential operators on $G$ ).

LEMma 3.1. $S^{p} \subseteq L^{r}$ for any real number $r \geqq p$. 
Proof. By (3.1) $\left|f\left(k g_{\zeta} \kappa\right)\right|^{r}=O\left(e^{-2 \zeta r / p} /\left(1+|\zeta|^{j}\right)\right)$. By the expression $d\left(k g_{5} \kappa\right)=\sinh 2 \zeta d \zeta d k d \kappa$ for the Haar-measure, the result follows for $r \geqq p$.

We now define a topology on $S_{m n}^{p}$ by the semi-norms

$$
\sup _{\boldsymbol{k}, \kappa, \zeta}\left(1+|\zeta|^{j}\right) e^{2 \zeta / p}\left|(\partial f)\left(k g_{\zeta} \kappa\right)\right| ;
$$

for every integer $j \geqq 0$ and every $\partial$ as above. This family of semi-norms is used to define the topology of $S_{m n}^{p}$. We can easily prove:

Lemma 3.2. $S_{m n}^{p}$ is a complete metrizable locally convex topological vectorspace. It is also a Schwartz space in the sense of Grothendieck [1].

Lemma 3.3. For fixed $m$ and $n$, and $0 \leqq R s \leqq 1$,

$$
\left|\frac{\partial^{j}}{\partial s^{j}} u_{m n}\left(g_{\zeta}, s\right)\right| \leqq \text { const } \zeta^{j} e^{-2 \zeta\{\min (\sigma, 1-\sigma)\}}
$$

where the currstant is independent of $m, n$ and $\zeta$. If $s$ is in a strip $-\epsilon \leqq R s$ $\leqq 1+\epsilon$ then the constant can be chosen independent of $s$.

Proof. We have (see Part 1, pp. 408-409)

$$
u_{m n}\left(g_{\zeta}, s\right)=\int_{0}^{1} e^{-2 \pi i m \sigma_{\zeta} \theta}\left|\frac{d g_{\zeta} \theta}{d \theta}\right|^{s} e^{2 \pi i n \theta} d \theta
$$

so that

$$
\begin{aligned}
\left|\frac{\partial^{j}}{d s^{j}} u_{m n}\left(g_{\zeta}, s\right)\right| \leqq & \left.\int_{0}^{1}\left|\frac{\partial^{j}}{\partial s^{j}}\right| \frac{d g_{\zeta} \theta}{d \theta}\right|^{8} \mid d \theta \\
= & \int_{0}^{1} \mid \frac{\partial^{j}}{\partial s^{j}} \exp \left\{-2 s \log \left|\cosh \zeta+e^{2 \pi i \theta} \sinh \zeta\right|\right\} d \theta \\
= & 2^{j} \int_{0}^{1}\left[\log ^{j}\left|\cosh \zeta+e^{2 \pi i \theta} \sinh \zeta\right|\right] \\
& -\exp \left\{-2 s \log \left|\cosh \zeta+e^{2 \pi i \theta} \sinh \zeta\right|\right\} d \theta
\end{aligned}
$$

Hence,

$$
\begin{aligned}
\left|\frac{\partial^{j}}{\partial s^{j}} u_{m n}\left(g_{\zeta}, s\right)\right| & \leqq(2 \zeta)^{j} \int_{0}^{1} \exp \left(-2 \sigma \log \left|\cosh \zeta+e^{2 \pi i \theta} \sinh \zeta\right|\right\} d \theta \\
& =(2 \zeta)^{j} u_{00}\left(g_{\zeta}, \sigma\right) .
\end{aligned}
$$

Now we use (4.9) of Part I (p. 420) whereby we may write $\left(u_{00}\left(g_{\zeta}, s\right)\right.$ $=\phi(\zeta, s))$,

$$
\phi(\zeta, s)=\phi^{(1)}(\zeta, s)+\phi^{(2)}(\zeta, s)
$$

where $\phi^{(2)}(\zeta, s)=\phi^{(2)}(\zeta, 1-s)$ and, by Equation (4.10) of Part I, $\left(y=\sinh ^{2} \zeta\right)$ 


$$
\phi^{(1)}(\zeta, s)=y^{-s} \frac{\sin ^{2} \pi s}{\pi \sin 2 \pi s} \int_{0}^{1} \tau^{s-1}(1-\tau)^{-1}(1-\tau / y)^{-s} d \tau
$$

Thus, for $-\epsilon \leqq \sigma \leqq 3 / 2-\epsilon$, we obtain

$$
\left|(s-1 / 2) \phi^{(1)}(\zeta, s)\right| \leqq \text { const }(1+|s|) e^{-2 \zeta \sigma}
$$

where the constant is independent of $s$. Hence, by the above

$$
|(s-1 / 2) \phi(\zeta, s)| \leqq \text { const }(1+|s|) e^{-2 \zeta\{\min (\sigma, 1-\sigma)\}}
$$

where the constant is independent of $s$ as long as $-\epsilon \leqq R s \leqq 1+\epsilon$. The lemma follows from this and the fact that $\phi(\zeta, s)$ is analytic in $s$.

By the above lemma, given any $f \in S_{m n}^{p}$, the integral $\int f(g) u_{m n}(g, s) d g$ converges uniformly for $s$ in the strip $1 / q \leqq R s \leqq 1 / p$ and defines, in fact a function $F(s)=\left(\mathfrak{I}_{0} f\right)(s)$ which is indefinitely differentiable and bounded in the closed strip and analytic in the interior. (If $p=2$, the strip degenerates to the line $R s=1 / 2$ and $F(1 / 2+i t)$ is a bounded indefinitely differentiable function of $t$.) Also if $\Delta$ denotes the Laplacian on $G$, then $\Delta^{i} f \in S_{m n}^{p}$ for any $j$ by the definition of $S_{m n}^{p}$. Moreover, by the self-adjointness of $\Delta$, we derive easily,

$$
\begin{aligned}
\int\left(\Delta^{i} f\right)(g) u_{m n}(g, s) d g & =\int f(g) \Delta^{i} u_{m n}(g, s) d g \\
& =s^{j}\left(1-s^{j}\right) F(s) .
\end{aligned}
$$

Thus, by our above remarks $\left(d^{r} / d s^{r}\right)\left[s^{i}\left(1-s^{j}\right) F(s)\right]$ is for every $r \geqq 1$ bounded in the strip $1 / q \leqq R s \leqq 1 / p$. It is clear from the definitions and from formulas (3.4) of Part I that $F(1-s)=\chi_{m n}(s) F(s)$ where $\chi_{m n}(s)$ is defined in Part I, p. 414 , formulas (3.5).

For any $f \in S_{m n}^{p}$ we define $\mathfrak{I} f$ as the triple $\mathfrak{T} f=\left(F(s), f^{+}(l), f^{-}(l)\right)$ where

$$
f^{+}(l)=\int f(g) v_{m n}^{+}(g, l) d g \quad \text { for } l=1,2,3, \cdots, \min (m, n)+1
$$

and

$$
\overrightarrow{f^{-}}(l)=\int f(g) v_{m n}^{-}(g, l) d g \quad \text { for } l=1,2,3, \cdots, \min (-m,-n)+1 .
$$

Here $\vartheta_{m n}^{ \pm}(g, l)$ denote again the matrix coefficients of the discrete series of irreducible unitary representations (cf. (1.5)-(1.10) of Part II).

Let us denote by $\widetilde{S}_{m n}^{p}$ the space of triples $\left\{H(s), h^{+}(l), h^{-}(l)\right\}$ where $H(s)$ is an analytic function in $1 / q<R s<1 / p$ and indefinitely differentiable in the closure of this strip; $H(1-s)=\chi_{m n}(s) H(s)$, and for any $j, r,(1+|s| r)\left(d^{j} H / d s^{j}\right)$ $\cdot(s)$ is uniformly bounded in the closed strip; and where $h^{+}(l)$ is a finite sequence of complex numbers defined for $l=1, \cdots, 1+\min (m, n)$ and $h^{-}(l)$ 
is defined for $l=1, \cdots, 1+\min (-m,-n)$; and where, for $p=1, H(0)$ $=H(1)=0$ if $m \geqq 0$ and $n<0$, or if $m \leqq 0, n>0\left({ }^{2}\right)$. We define in $\tilde{S}_{m n}^{p}$ a locally convex topology by means of the semi-norms

$$
\sup _{l, 1 / q \leqq R s \leqq 1 / p}\left\{\left|h^{+}(l), h^{-}(l),\left(1+|s|^{r}\right)\right|\left(\frac{d^{i}}{d s^{j}} H\right)(s) \mid\right\}
$$

for all integers $r, j \geqq 0$. It is readily verified that $\widetilde{S}_{m n}^{p}$ is a complete, metrizable, locally convex topological vector space which is a Schwartz space (see Grothendieck [1]).

THEOREM 3.1. $\mathfrak{T}$ is a topological isomorphism of $S_{m n}^{p}$ onto $\widetilde{S}_{m n}^{p}$.

Proof. We have shown above that $\mathfrak{T}$ maps $S_{m n}^{p}$ into $\tilde{S}_{m n}^{p}$. (In case $p=1$ we use Lemma 1.4 of Part II and the above.) Since $\mathfrak{I}$ is linear, we need prove continuity only at 0 . Suppose $f^{j} \rightarrow 0$ in $S_{m n}^{p}$. Then clearly $f^{j} \rightarrow 0$ in $L^{2}$; since for each $l$ we have $v_{m n}^{ \pm}(g, l) \in L^{2}$, it follows that for each $l, f^{i \pm}(l) \rightarrow 0$. Also, for any non-negative integers $a$ and $b$, we have, by the definition of the topology of $S_{m n}^{p}$,

$$
e^{2 \zeta / p}\left(1+\zeta^{a}\right)\left|\left(\Delta^{b} f^{j}\right)\left(g_{\zeta}\right)\right| \rightarrow 0
$$

uniformly in $\zeta$. It follows thus from Lemma 3.3 and the expression $d g$ $=d k \sinh 2 \zeta d \zeta d \kappa$ that, for any $a^{\prime}, b^{\prime}$, we have

$$
\left(1+|s|^{a^{\prime}}\right)\left|\left(\frac{d^{b^{\prime}}}{d s^{b^{\prime}}} F^{j}\right)(S)\right| \rightarrow 0
$$

uniformly for $1 / q \leqq R s \leqq 1 / p$. Thus, $\mathfrak{T} f^{j} \rightarrow 0$ in $\widetilde{S}_{m n}^{p}$ so $T$ is continuous.

To prove that $\mathfrak{I}$ is onto and that $\mathfrak{T}^{-1}$ is continuous, we shall consider first the case $m=n=0$. In this case, if $\left\{H(s), h^{+}(l), h^{-}(l)\right\}$ is a triple in $\tilde{S}_{00}^{p}$, the terms $h^{+}(l)$ and $h^{-}(l)$ do not appear, so we may identify the triple $\left\{H(s), h^{+}(l), h^{-}(l)\right\}$ with the function $H(s)$ and $\widetilde{S}_{00}^{p}$ is identified with a space of functions $H(s)$.

The fact that $\mathfrak{I}$ is a topological isomorphism of $S_{00}^{1}$ onto $\widetilde{S}_{00}^{1}$ is proven in Part I, Theorem 4. (Note that $S_{00}^{1}$ and $\widetilde{S}_{00}^{1}$ were denoted in Part I by $S$ and $\hat{S}$ respectively.) We give now the modifications that must be made in case $1<p \leqq 2$. We shall write, as in Part I, $u_{00}\left(g_{\zeta}, s\right)=\phi(\zeta, s)$, and $y=\sinh ^{2} \zeta$. Then, as in formula 4.10 of Part I, we write $\phi(\zeta, s)=\phi^{(1)}(\zeta, s)+\phi^{(2)}(\zeta, s)$. Now, if $H(s) \in \widetilde{S}_{00}^{p}$, it follows from Lemma 3.3 that the integral $\int_{1 / 2-i \infty}^{1 / 2+\infty \infty} H(s) \phi^{(1)}(g, s) \delta s$ where $\delta s=i(1 / 2-s)$ tan $[\pi(1 / 2-s)] d s$ defines a continuous function $h(g)$ which is spherical of type $(0,0)$. Moreover, $h(g) \in L_{2}(G)$ and, as in Part I, pp. $421-422, H(s)=\int h(g) \phi^{(1)}(g, s) d g=(T h)(s)$.

We have to show first that $h \in S_{00}^{p}$. We write

(2) In case $p=1, h^{ \pm}(1)=w_{m n}(1) H(1)$ for those $m, n$ for which $h^{+}(1)$ or $h^{-}(1)$ is defined (cf. (1.9) and (1.10) of Part II). 


$$
\begin{aligned}
\psi^{(1)}(y, s)= & (i / 2)(1 / 2-s) \tan [\pi(1 / 2-s)]\left(\sin ^{2} \pi s / \pi \sin 2 \pi s\right) \\
& \cdot \int_{0}^{1}(1-\tau)^{s-1} \tau^{s-1}(1-\tau / y)^{-8} d \tau .
\end{aligned}
$$

Then, using (4.10) of Part I, p. 420, we have

$$
h(y)=\int_{1 / 2-i \infty}^{1 / 2+i \infty} H(s) y^{-s} \psi^{(1)}(y, s) d s
$$

where $y=\sinh ^{2} \zeta$. We now use the fact that $H(s)$ is in $\tilde{S}_{00}^{p}$, so $H(s)$ is analytic in the strip $1 / q<R s<1 / p$ and rapidly decreasing in the closed strip $1 / q \leqq R s$ $\leqq 1 / p$. On the other hand, inequality (4.11) of Part I, p. 421 , shows us that, for $y \geqq 2,1 / 4 \leqq R s \leqq 1$, we have $\psi^{(1)}(y, s) \leqq B_{0}|s(1-s)|$ for some $B_{0}>0$. Thus, we can shift the contour in (3.4) if $y \geqq 2$ and we obtain

$$
h(y)=\int_{1 / p-i \infty}^{1 / p+i \infty} H(s) y^{-s} \psi^{(1)}(y, s) d s .
$$

Now, we have shown in Part I, pp. 419-420, that, given any $\partial$ which is a polynomial in the elements of the Lie algebra of $G$ and any compact set $C$ in $G$, we can find a polynomial $P(s)$ such that

$$
|(\partial \phi)(g, s)| \leqq|P(s)|
$$

whenever $g \in C$ and $0 \leqq R s \leqq 1$. Thus, $\int_{1 / 2-\imath_{\infty}}^{1 / 2+4 \infty} H(s)(\partial \phi)(g, s) \delta s$ converges uniformly in compact sets of $G$ for $H \in \widetilde{S}_{00}^{p}$. This means that $h(g)$ is indefinitely differentiable and, using (6.26) of Bargmann [1] and Lemma 2.6 of Part II, we can find a polynomial $Q(s)$ such that

$$
(\partial h)(g)=\int_{1 / 2-i \infty}^{1 / 2+i \infty} Q(s) H(s) \phi(g, s) \delta s .
$$

Since $s^{r}(1-s)^{r} H(s)$ is in $\widetilde{S}_{00}^{p}$ whenever $H \in \widetilde{S}_{00}^{p}$, formula (3.95) shows us that

$$
(\partial h)(g)=\int_{1 / p-i \infty}^{1 / p+i \infty} Q(s) H(s) \phi(g, s) \delta s .
$$

We shall now use the following analog of Lemma 4.1 of Part I, p. 422. The proof is exactly the same as the proof of Lemma 4.1 as given in Part I, p. 423:

Lemma 3.4. (a) $h(y)$ is bounded. Moreover we can find an $N>0$ so that the condition $|H(s)| \leqq \epsilon /\left(H|s|^{8}\right)$ whenever $1 / q \leqq R s \leqq 1 / p$ implies $|h(y)| \leqq \epsilon N$ for all $y$.

(b) For any $r>0$ we can find a $B_{r 0}>0$ so that if

$$
H(s) \leqq \epsilon /\left(1+|s|^{8}\right), \cdots,\left|\left(d^{r} / d s^{r}\right) H(s)\right| \leqq \epsilon /\left(1+|s|^{8}\right) .
$$


Whenever $1 / q \leqq R s \leqq 1 / p$, then

$$
\left|y^{1 / p} \log ^{r} y h(y)\right| \leqq \epsilon B_{r 0}
$$

for all $y \geqq 2$.

By use of the lemma for $Q(s) H(s)$ in place of $H(s)$, we find that

$$
y^{1 / p} \log ^{r} y \partial h(y) \text { and } \partial h(y)
$$

are bounded functions. Now, for $y$ large, $y=\sinh ^{2} \zeta \sim e^{25}$, so that $e^{2 \zeta / p}\left(1+|\zeta|^{r}\right) \partial h(\zeta)$ is bounded for all $\zeta$. This means that $h \in S_{00}^{p}$.

Also, by making

$$
|Q(s) H(s)| \leqq \epsilon /\left(1+|s|^{3}\right), \cdots,\left|\left(d^{r} / d s^{r}\right) Q(s) H(s)\right| \leqq \epsilon /\left(1+|s|^{3}\right)
$$

we can be assured that

$$
\left|y^{1 / p} \log ^{r} y \partial h(y)\right| \leqq \epsilon B_{r 0} \quad \text { for } y \geqq 2
$$

and

$$
|\partial f(\zeta)| \leqq \epsilon N \quad \text { for all } \zeta .
$$

This, in turn, implies the existence of a positive constant $B_{r 0}^{\prime}$ so that

$$
(1+|\zeta| r) e^{2 \zeta / p}|h(\zeta)| \leqq \epsilon B_{r 0}^{\prime}
$$

which means that $\mathfrak{I}^{-1}$ is continuous at zero. Since $\mathfrak{T}^{-1}$ is clearly linear, it is continuous. Thus, $\mathfrak{I}$ is a topological isomorphism of $S_{00}^{p}$ onto $\widetilde{S}_{00}^{p}$.

In case $p=1$, we assume first that $H(0)=H(1)=0$ if $(m, n) \neq(0,0)\left({ }^{3}\right)$.

Let us now consider the case of $m, n$ arbitrary. For $\left\{H(s), h^{+}(l), h^{-}(l)\right\}$ $\in \tilde{S}_{m n}^{p}$, set

$$
h_{ \pm}(g)=\sum_{l} h^{ \pm}(l) v_{m n}^{ \pm}(g, l)[2 l-1]^{1 / 2} .
$$

From the expression (11.2) of Bargmann [1] for the $v_{m n}^{ \pm}(g, l)$ and the fact that $\Delta v_{m n}^{ \pm}(g, l)=l(1-l) v_{m n}^{ \pm}(g, l)$, we see that $h_{ \pm}(g) \in S_{m n}^{p}$ unless $p=l=1$ and, in fact, the maps $\left\{H(s), h^{+}(l), h^{-}(l)\right\} \rightarrow h_{ \pm}(g)$ are continuous linear maps of $\tilde{S}_{m n}^{p}$ into $S_{m n}^{p}$.

Set

where

$$
F(s)=H(s) / \eta_{m n}(s)
$$

$$
\text { (3.8) } \quad \eta_{m n}(s)= \begin{cases}\prod_{j=1}^{|n|}(j-s) \prod_{j=1}^{|m|}(j-1+s) & \text { if }|n| \geqq|m|, \\ \prod_{j=1}^{|n|}(j-1+s) \prod_{j=1}^{|m|}(j-s) & \text { if }|m| \geqq|n| .\end{cases}
$$

(3) So that, if $p=1, h^{ \pm}(1)=0$. 
Then it is clear that $F(s) \in \tilde{S}_{00}^{p}$ and that the map $\left\{H(s), h^{+}(l), h^{-}(l)\right\} \rightarrow F(s)$ is a continuous linear map of $\widetilde{S}_{m n}^{p}$ into $\tilde{S}_{00}^{p}$. Write $f=\left(\mathfrak{T}^{-1} F\right)$, so by our previous result for $\widetilde{S}_{00}^{p}$, we have $f \in S_{00}^{p}$ and $\left\{H(s), h^{+}(l), h^{-}(l)\right\} \rightarrow f(g)$ is a continuous linear map of $S_{m n}^{p}$ into $S_{00}^{p}$. By Lemma 2.6 of Part II, we can find an $\epsilon_{m n}$ which is a polynomial in left and right derivatives on $G$ (by elements of the Lie algebra of $G$ ) such that $h_{0}=\epsilon_{m n} f$.

We need the following

\section{Lemma 3.5. The map $f^{\prime} \rightarrow \epsilon_{m n} f^{\prime}$ is a continuous linear map of $S_{00}^{p}$ into $S_{m n}^{p}$.}

Let us assume Lemma 3.5 for the present and conclude the proof of Theorem 3.1 (with our restriction in case $p=1$ that $H(1)=H(0)=0)$. The above shows that $\left\{H(s), h^{+}(l), h^{-}(l)\right\} \rightarrow h_{0}(g)$ is a continuous linear map of $\tilde{S}_{m n}^{p}$ into $S_{m n}^{p}$. Now, let us set

$$
h(g)=h_{0}(g)+h_{+}(g)+h_{-}(g) .
$$

We have shown that $\left\{H(s), h^{+}(l), h^{-}(l)\right\} \rightarrow h(g)$ is a continuous linear map of $\tilde{S}_{m n}^{p}$ into $S_{m n}^{p}$. On the other hand, it follows from Lemma 2.6 of Part II that $\mathfrak{T} h=\left\{H(s), h^{+}(l), h^{-}(l)\right\}$. This concludes the proof of Theorem 3.1. It remains to prove Lemma 3.5.

Proof of Lemma 3.5. The topology of $S_{m n}^{p}$ is defined in terms of the seminorms (3.2). Since $\epsilon_{m n}$ is a differential operator on $G$ obtained from the Lie algebra of $G$ (considered as both left and right differential operators) by taking polynomials, the result is immediate.

The case $p=1$ concluded. We assume now that $H(0)$ or $H(1) \neq 0$ (which can occur for only certain values of $(m, n)$ by the definition of $\left.\tilde{S}_{m n}^{1}\right)$. To conclude the proof for $p=1$, we note that in Part II (see the final step in the proof of Theorem 2.1 of Part II) we showed that, for any $H(s) \in \tilde{S}_{m n}^{1}$ we can find an $H_{1}(s) \in \mathfrak{I}_{0} D_{m n}$ such that

$$
H(1)-H(1) H_{1}(1)=H(0)-H(1) H_{1}(0)=0 .
$$

Thus, $H(s)-H(1) H_{1}(s)=H^{1}(s)$ satisfies our previous condition, and the result follows easily from the above and Theorem 2.1 of Part II.

Corollary. For any $m, n, r$, let $f \in S_{m n}^{p}$ and $h \in S_{n r}^{p}$. Then $(f, h) \rightarrow f * h$ is a continuous linear map of $S_{m n}^{p} \times S_{n r}^{p}$ into $S_{m r}^{p}$.

Proof. Let $f^{i}$ be a sequence in $D_{m n}$ such that $f^{j} \rightarrow f$ in $S_{m n}^{p}$. Write $\mathfrak{T} f^{i}$ $=\left\{F^{j}(s), f^{i+}(1), f^{j-}(1)\right\}, \mathfrak{I} f=\left\{F(s), f^{+}(l), f^{-}(l)\right\}, \mathfrak{T} h=\left\{H(s), h^{+}(l), h^{-}(l)\right\}$. Then (see e.g. Part II) $\mathfrak{T}\left(f^{j} * h\right)=\left\{F^{i}(s) H(s), f^{j+}(l) h^{+}(l), f^{j-}(l) h^{-}(l)\right\}$. By Theorem 3.1 and the definition of the topologies of $\tilde{S}_{m n}^{p}, \widetilde{S}_{n r}^{p}, \widetilde{S}_{m r}^{p}$, it follows that $T\left(f^{j} * h\right)$ converges to $\left\{F(s) H(s), f^{+}(l) h^{+}(l), f^{-}(l) h^{-}(l)\right\}$ in the topology of $\tilde{S}_{m r}^{p}$. Since $f^{j *} h \rightarrow f^{*} h$ in the topology of $D$, it follows that $T\left(f^{*} h\right)=\{F(s) H(s)$, $\left.f^{+}(l) h^{+}(l), f^{-}(l) h^{-}(l)\right\}$. The continuity of the map $(f, h) \rightarrow f^{*} h$ now follows from Theorem 3.1 and the definition of the topology of $\widetilde{S}_{m n}^{p}$. 
Corollary. For any $m, S_{m m}^{p}$ is a topological algebra under convolution.

We now define for each fixed $p \geqq 1$, $\leqq 2$ the group algebra $S^{p}$ as the weak direct sum of the spaces $S_{m n}^{p}$ as follows: Let $f(g)$ be any (continuous) complexvalued function on $G$. We let

$f \in S^{p}$ if and only if $P_{m n} f \in S_{m n}^{p}$ for all $m, n$ and $P_{m n} f=0$ for all but a finite number of $m, n$.

We define a topology on $S^{p}$ by considering for each pair of integers $M, N>0$ the finite direct sum $S^{p, M, N}=\sum_{|m| \leq M,|n| \leq N} S_{m n}^{p}$ of the spaces $S_{m n}^{p}$ with the Cartesian product topology. Clearly $S^{p}$ is the limit of these spaces as $M \rightarrow \infty$ and $N \rightarrow \infty$ and we define the topology of $S^{p}$ to be the inductive limit topology in the sense of Dieudonné and Schwartz [1] of the spaces $S^{p, M, N}$.

Proposition 3.1. For each $p \geqq 1$ and $\leqq 2, S^{p}$ is a topological algebra with convolution as product. Under addition of functions it is a locally convex metrizable complete topological vector space. The projection $P_{m n}: S^{p} \rightarrow S_{m n}^{p}$ is for each $m, n$ a continuous linear mapping.

This is an immediate consequence of Proposition 5 of Dieudonné and Schwartz [1] together with the above properties of the spaces $S_{m n}^{p}$.

We next define the algebra $\tilde{S}^{p}$ to consist of all triples of matrices $\left\{H_{m n}(s), h_{m n}^{+}(l), h_{m n}^{-}(l)\right\}$ which are for each fixed $m, n$ contained in the above space $\widetilde{S}_{m n}^{p}$. We assume that $H_{m n}(s)$ is identically zero in $s$ and $h_{m n}^{ \pm}(l)$ is identically zero in $l$ for all but a finite number of pairs $m, n$. We put on $\tilde{S}_{p}$ the inductive limit topology of the spaces $\sum_{|m| \leq M,|n| \leq N} \widetilde{S}_{m n}^{p}$. We make $\widetilde{S}^{p}$ into an algebra by defining sum point-wise (i.e. by addition for each $s, l, m, n$ ) while the product is defined by matrix-multiplication for each $s$ and $l$ :

$$
\begin{aligned}
\left\{A_{m n}(s), a_{m n}^{+}(l), a_{m n}^{-}(l)\right\} & \cdot\left\{B_{m n}(s), b_{m n}^{+}(l), b_{m n}^{-}(l)\right\} \\
& =\left\{\sum_{j} A_{m j}(s) B_{j n}(s), \sum_{j} a_{m j}^{+}(l) b_{j n}^{+}(l), \sum_{j} \overline{a_{m j}}(l) b_{j n}^{-}(l)\right\} .
\end{aligned}
$$

From the above properties of the $\widetilde{S}_{m n}^{p}$ and the properties of an inductive limit one obtains

Proposition 3.2. $\tilde{S}^{p}$ is a topological algebra. It is a locally convex metrizable complete topological vector space. The projection $\tilde{S}^{p} \rightarrow \tilde{S}_{m n}^{p}$ is for each $m, n$ a continuous linear mapping.

Using again the above results and the properties of an inductive limit we have

TheOREM 3.2. The mapping $\mathfrak{T}$ is a topological isomorphism of $S^{p}$ onto $\tilde{S}^{p}$.

Let $C \widetilde{S}^{p}$ be the set of all those triples $\left\{H_{m n}(s), h_{m n}^{+}(l), h_{m n}^{-}(l)\right\} \in \tilde{S}^{p}$ for 
which $h_{m n}^{ \pm}(l)=0$ for all $l, m, n$. It is clear that $C \tilde{S}^{p}$ is a closed two-sided ideal of $\widetilde{S}^{p}$. Let $\tilde{S}_{+}^{p}$ be the set of all those triples $\epsilon \widetilde{S}^{p}$ for which $H_{m n}(s)=0$ and $h_{m n}^{-}(l)=0$ identically. Similarly $\tilde{S}_{-}^{p}$ is defined by $H_{m n}(s)=0$ for all $s, m, n$ and $h_{m n}^{+}(l)=0$ for all $m, n, l$. Clearly $\widetilde{S}_{+}^{p}$ and $\tilde{S}_{-}^{p}$ are closed two-sided ideals of $\widetilde{S}^{p}$ and

$$
\tilde{S}^{p}=C \tilde{S}^{p} \oplus \tilde{S}_{+}^{p} \oplus \tilde{S}_{-}^{p} .
$$

We write $C S^{p}=T^{-1} C \tilde{S}^{p}, S_{ \pm}^{p}=T^{-1} \tilde{S}_{ \pm}^{p}$ and have

$$
S^{p}=C S^{p} \oplus S_{+}^{p} \oplus S_{-}^{p}
$$

We denote $C \tilde{S}^{p} \cap \tilde{S}_{m n}^{p}$ by $C \tilde{S}_{m n}^{p}$.

Lemma 3.6. Let $J$ be a closed two-sided ideal of $C \tilde{S}^{p}$. If $1<p \leqq 2$ the mapping

$$
J \rightarrow J \cap C \tilde{S}_{00}^{p}
$$

is a 1-1 correspondence between all closed two-sided ideals of $C \tilde{S}^{p}$ and all closed ideals of the commutative algebra $C \tilde{S}_{00}^{p}$.

Proof. Consider the functions $\eta_{m n}(s)$ defined by (3.8) above. Let $J_{00}$ be a closed ideal of the algebra $C \widetilde{S}_{00}^{p}$. The elements of $C \widetilde{S}_{00}^{p}$ are certain functions $H(s)=H(1-s)$. It is clear from the definitions of $\tilde{S}_{m n}^{p}$ and $\eta_{m n}(s)$ that if $H(s) \in C \tilde{S}_{00}^{p}$ then $\eta_{m 0}(s) \eta_{0 n}(s) H(s) \in C \tilde{S}_{m n}^{p}$ for each $m, n$. As $H(s)$ varies over $J_{00}$, the functions $\eta_{m 0}(s) \eta_{0 n}(s) H(s)$ vary for fixed $m, n$ over a closed leftsubmodule of $C \tilde{S}_{m n}^{p}$ (over $C \tilde{S}_{m n}^{p}$ ) which we denote by $J_{m n}$.

Now given a closed two-sided ideal $J$ of $C \widetilde{S}^{p}$, put $J_{00}=J \cap C \widetilde{S}_{00}^{p}$. Let $J_{m n}=\eta_{m 0}(s) J_{00} \eta_{0 n}(s)$. Let $\Im$ be the closed two-sided ideal generated by $J_{00}$ in $C \tilde{S}^{p}$. Write $\Im_{m n}=\Im \cap C \widetilde{S}_{m n}^{p}$. Clearly $\Im \subseteq J$, hence $\Im_{m n} \subseteq J_{m n}$ for each $m, n$. We wish to prove that $J_{m n} \subseteq \Im_{m n}$. It is enough to show that $J_{m n} \subseteq \Im$.

By an easy approximation argument it follows that $\eta_{m n}(s)$ can be approximated by elements of $C \tilde{S}_{m n}^{p}$ in such a manner that $\eta_{m 0}(s) J_{00} \eta_{0 n}(s) \subseteq \Im$, i.e. $J_{m n} \subseteq \Im$. This proves that $\Im_{m n}=J_{m n}$ for all $m, n$.

We prove next that $J_{m n}=J \cap C \tilde{S}_{m n}^{p}$. Since $\eta_{m n}(s) J \subseteq J$, so $J_{m n}$ $=\eta_{m 0}(s) J_{00} \eta_{0 n}(s) \subseteq J$. Thus $J_{m n} \subseteq J \cap C \tilde{S}_{m n}^{p}$. Now $\eta_{m n}(s)$ has no zeros in the strip $0<R s<1$, so that $\eta_{m n}(s)^{-1}$ is regular in this strip and bounded, so that it can be approximated by elements of $C \tilde{S}_{m n}^{p}$ so that $\eta_{0 m}(s)^{-1}\left(J \cap C \widetilde{S}_{m n}^{p}\right) \eta_{n 0}(s)^{-1}$ $\subseteq J_{00}$. We conclude that $J_{m n}=J \cap C \tilde{S}_{m n}^{p}$.

Let $\mathcal{E}^{j}$ be the matrix whose $m, n$th component is $\delta_{j m} \delta_{j n}$. We can approximate the function 1 by functions $\epsilon C \widetilde{S}_{m m}^{p}$ so that $\mathcal{E}^{m} J \subseteq J$ for any closed twosided ideal of $C \tilde{S}^{p}$. Also clearly $\mathcal{E}^{m}\left(C \widetilde{S}^{p}\right) \mathcal{E}^{n} \subseteq C \tilde{S}_{m n}^{p}$. Thus $\mathcal{E}^{m} J \mathcal{E}^{n} \subseteq J \cap C \widetilde{S}_{m n}^{p}$. On the other hand $J \cap C \widetilde{S}_{m n}^{p} \subseteq \mathcal{E}^{m} J \mathcal{E}^{n}$ is obvious. Thus $\mathcal{E}^{m} J \mathcal{E}^{n}=J \cap C \widetilde{S}_{m n}^{p}$ $=J_{m n}$ and $\mathcal{E}^{m} \Im_{\mathcal{E}^{n}}=\Im_{m n}$.

We have already seen above that $\Im_{m n}=J_{m n}$ for all $m, n$, hence $\mathcal{E}^{m} \Im_{\mathcal{E}^{n}}$ $=\mathcal{E}^{m} J \mathcal{E}^{n}$ for all $m, n$. This implies that $\Im=J$. 
Proposition 3.3. Every proper closed ideal $J$ of the commutative topological algebra $S_{00}^{2}$ is the intersection of the primary closed ideals containing it.

By a primary closed (left, right, two-sided) ideal we mean a (left, right, two sided) closed ideal which is is contained in one (left, right, two-sided) maximal closed ideal.

Proof. Let $\subseteq$ be the algebra of all indefinitely differentiable functions of one real variable $t$ which are rapidly decreasing (i.e. every derivative is $O\left(|t|^{j}\right)$ for all $\left.j\right)$. In the topology of Schwartz $\mathfrak{S}$ is a topological algebra with point-wise multiplication as product. By a theorem of Whitney [1] every closed proper ideal of $\mathfrak{S}$ is determined by its zeros with multiplicities.

Now given any $h \in S_{00}^{2}$; its Fourier transform $H(1 / 2+i t)=H(1 / 2-i t)$ is an even function of $t$ contained in $\widetilde{S}_{00}^{2}$ and $\widetilde{S}_{00}^{2} \subseteq \subseteq$ and the topology of $\widetilde{S}_{00}^{2}$ is the same as that induced by $\mathfrak{S}$.

Now given any proper closed ideal $J$ of $\tilde{S}_{00}^{2}$, consider the closed ideal $J \subseteq$ generated by $J$ in $\subseteq$. If $F \in J \subseteq \cap \widetilde{S}_{00}^{2}$ then $F=\lim \sum H_{\alpha} A_{\alpha}$ where $H_{\alpha} \in J$ and $A_{\alpha} \in \Im$. Then $F(1 / 2+i t)=\lim \sum H_{\alpha}(1 / 2+i t) 2^{-1}\left\{A_{\alpha}(1 / 2+i t)+A_{\alpha}(1 / 2-i t)\right\}$. Put $B_{\alpha}(1 / 2+i t)=2^{-1}\left\{A_{\alpha}(1 / 2+i t)+A_{\alpha}(1 / 2-i t)\right\}$. Then $B_{\alpha} \in \tilde{S}_{00}^{2}$ and $F$ $=\lim \sum H_{\alpha} B_{\alpha}$. Thus $F \in J$. This proves that $J \subseteq \cap \widetilde{S}_{00}^{2}=J$. Now if $J_{1}$ is another closed ideal of $\tilde{S}_{00}^{2}$ with the same zeros and multiplicities as $J$, then by the above mentioned theorem of Whitney [1] $J \subseteq=J_{1} \subseteq$. Hence $J=J_{1}$.

Now the maximal closed ideals of $\tilde{S}_{00}^{2}$ are given by the pair of points $1 / 2+i t, 1 / 2-i t$ as follows easily from the knowledge of all maximal ideals of ऽ. It follows that each primary ideal of $S_{00}^{2}$ consists of all those $h \in S_{00}^{2}$ for which $H(1 / 2+i t)$ vanishes at one point $t_{0}$ up to a given order. This proves Proposition 3.3.

As an immediate consequence of Proposition 3.3 and Lemma 3.6 we have

Corollary. Every closed proper two-sided ideal of the algebra $\mathrm{CS}^{2}$ is the intersection of the two-sided primary closed ideals which contain it.

We next consider the two-sided ideals of $\widetilde{S}_{+}^{p}$ for $1<p \leqq 2$. For each $l \geqq 2$ let $\tilde{S}_{+}^{p}(l)$ be the set of $h_{m n}^{+}(l)$ for $h^{+} \in S_{+}^{p}$. Thus, $\widetilde{S}_{+}^{p}(l)$ is the algebra of matrices $a_{m n}$ of complex numbers for $m, n \geqq l$, where $a_{m n}=0$ except for a finite number of $m$ and $n$. It is readily verified that the algebra $\tilde{S}_{+}^{p}(l)$ is simple.

Now, let $\Im$ be any two-sided ideal in $\widetilde{S}_{+}^{p}$; by $\Im(l)$ we denote $\Im \cap \widetilde{S}_{+}^{p}(l)$, so $\Im(l)$ is an ideal in $\widetilde{S}_{+}^{p}$ which is contained in $\Im$. By the simplicity of $\widetilde{S}_{+}^{p}(l)$, $\Im(l)=\{0\}$, or $\Im(l)=\widetilde{S}_{+}^{p}(l)$. If $\Im(l)=\{0\}$, we say that $\Im$ is zero at $l$.

We claim that $\Im$ is determined by its zeros. For, suppose that $J$ is another two-sided ideal in $\widetilde{S}_{+}^{p}$ which has the same zeros as $\Im$ and let $h^{+} \in J$. Then $h_{m n}^{+}(l)=0$ for all $l$ except for a finite number of $m$ and $n$; hence, $h_{m n}^{+}(l)=0$ for all $m$ and $n$ if $l$ is large enough, say if $l \geqq L$. Now, for any $l, h^{+}(l) \in \Im$ because $\Im(l)=\widetilde{S}_{+}^{p}(l)$ if $h^{+}(l) \neq 0$. Now, clearly, $h^{+}=\sum_{l<L} h^{+}(l)$, so that we have $h^{+} \in \Im$ since each $h^{+}(l) \in \Im(l) \subset \Im$. This proves that $J \subset \Im$; by similar reasoning, $\Im \subset J$, so $\Im=J$. 
A similar argument can be applied to the algebra $\tilde{S}_{-}^{p}$ for $1<p \leqq 2$.

Proposition 3.4. Let $1<p \leqq 2$. Then every two-sided ideal in $\tilde{S}_{+}^{p}$ or $\widetilde{S}_{-}^{p}$ is closed and is determined by its zeros. Thus, every two-sided ideal in $\widetilde{S}_{+}^{p} \oplus \tilde{S}_{-}^{p}$ is the intersection of the maximal two-sided ideals which contain it.

Proof. By the above, every two-sided ideal $\Im$ in $\widetilde{S}_{+}^{p}$ is determined by its zeros. It follows easily that $\Im$ is closed. It is also readily verified that the only maximal two-sided ideals in $\tilde{S}_{+}^{p}$ are the ideals $J_{l}$ consisting of all $h^{+} \in \tilde{S}_{+}^{p}$ with $h^{+}(l)=0$. A similar result holds for $\tilde{S}_{-}^{p}$. Proposition 3.4 then follows immediately.

Combining Propositions 3.3 and 3.4 we obtain immediately

TheOREm 3.3. Every proper closed two-sided ideal of the group algebra $S^{2}$ is the intersection of the two-sided primary closed ideals which contain it.

4. Mean periodic functions of fixed type $m, n$. In $\S 5$ of Part II we have studied mean periodic functions $\epsilon E_{m n}$. We shall now derive further properties of such functions.

Lemma 4.1. Let $f \in E_{m n}$ be mean periodic in the sense of $\$ 5$ of Part II. Let

$$
f(g)=\sum_{j} \sum_{q=1}^{r_{j}} d_{j q} u_{m n}^{(q)}\left(g, S_{j}\right)
$$

be the mean-periodic expansion of $f(g)$ obtained in $\$ 5$ of Part II. (Here the sum $\sum \sum$ is in the sense of the summability explained at the end of $\$ 5$ of Part II.)

Assertion: For each $j$ there exists an element $W_{j} \in E_{m m}^{\prime}$ such that

$$
\sum_{q=1}^{r_{i}} d_{j q} u_{m n}^{(q)}\left(g, s_{j}\right)=\left(W_{j} * f\right)(g) .
$$

Here $W_{j}$ is given by the expression

$$
\left(\mathfrak{I}_{0} W_{j}\right)(s)=\left\{\frac{1}{\mathfrak{T}_{0} W}\right\}_{s_{j}}(s)\left(\mathfrak{T}_{0} W\right)(s),
$$

where $\left\{1 / \mathfrak{T}_{0} W\right\}_{s_{j}}(s)$ denotes the principal part of the Laurent expansion of the mermorphic function $\left[\left(\mathfrak{I}_{0} W\right)(s)\right]^{-1}$ at the point $s_{j}$. (Note that since $W \in E_{m m}^{\prime}$, its Fourier transform $\mathfrak{I}_{0} W$ is a numerical valued entire function of exponential type (cf. $\$ 4$ of Part II) denoted by $\left(\mathfrak{I}_{0} W(s)\right)$.

Proof. We denote again by $\hat{E}^{\prime}$ the topological vector space of Schwartz of all entire functions of $s$ of exponential type which are slowly increasing along $R s=1 / 2$. For any $f \in E_{m n}$ we define a continuous linear function (denoted again by $\mathfrak{I} f)$ on the space $\hat{E}^{\prime}$ by putting for any $H=H(s) \in \hat{E}^{\prime}$

$$
\mathfrak{I} f \cdot H=\mathfrak{I} f \cdot\left\{2^{-1} \eta_{m n}(s)[H(s)+H(1-s)]\right\},
$$


where $\eta_{m n}(s)$ is defined by formula (3.8) of $\S 3$ above. Note that the right side of (4.4) is well defined, since $2^{-1} \eta_{m n}(s)[H(s)+H(1-s)]$ is contained in the space $\mathfrak{T}_{0} E_{m n}^{\prime}$ of $\S 4$ of Part II and $\mathfrak{I} f$ is in the dual of $\mathfrak{T}_{0} E_{m n}^{\prime}$. We have to show that $\mathfrak{I} f \cdot H$ is continuous on $\hat{E}^{\prime}$. It follows from Theorem 4.3 of Part II that $H(s) \rightarrow 2^{-1} \eta_{m n}(s)[H(s)+H(1-s)]$ is a linear map of $\hat{E}^{\prime}$ into $\mathfrak{I}_{0} E_{m n}^{\prime}$ which takes bounded sets into bounded sets. Since $\mathfrak{I} f$ is continuous on $\mathfrak{I}_{0} E_{m n}^{\prime}$, it follows that the linear function $H \rightarrow \mathfrak{T} f \cdot H$ is bounded on the bounded sets of $\hat{E}^{\prime}$. Since $\hat{E}^{\prime}$ is bornologic (see Grothendieck [1]), it follows that $\mathfrak{I} f \cdot H$ is continuous on $\hat{E}^{\prime}$.

Now we use the fact that $f(g)$ is mean periodic in $E_{m n}$. Hence by Proposition 5.1 of $\S 5$ of Part II there exists an element $W$ of $E_{m m}^{\prime}$ such that $W * f=0$. It follows that for any $H \in \hat{E}^{\prime}$

$$
T f \cdot\left\{\left(T_{0} W\right)(s) H(s)\right\}=0 .
$$

Hence we can apply the theory of L. Schwartz [3] and conclude that the linear function $\mathfrak{I} f$ on $\hat{E}^{\prime}$ has a formal expansion $\sum_{j} \sum_{g=1}^{r_{j}} d_{j_{q}} \delta_{s_{i}}^{(q)}$ where $\delta_{s_{j}}^{(q)}$ denotes the $q$ th derivative of the delta measure of the point $s_{j}$. Moreover

$$
\sum_{q=1}^{r_{j}} d_{j \delta \delta_{s_{j}}}^{(\boldsymbol{q})}=\left\{\frac{1}{\mathfrak{T}_{0} W}\right\}_{s_{j}}(s)\left(\mathfrak{T}_{0} W\right)(s)
$$

in accordance with formula (29) and (32) of L. Schwartz [3]. To prove the lemma we only have to apply $\mathfrak{T}^{-\mathrm{i}}$.

Lemma 4.2. Let $\alpha_{0}, \alpha_{1}, \cdots, \alpha_{r}$ be arbitrary given complex numbers. Let $s_{0}$ be a complex number with $R s_{0}>1 / p$. Then there exist entire functions $\psi_{\nu}(s)$ $=\psi_{\nu}(1-s)$ of exponential type, rapidly decreasing along $R s=1 / 2$ such that

$$
\lim _{\nu \rightarrow \infty}\left(1+|s|^{j}\right) \frac{d^{\mu} \psi_{\nu}}{d s^{\mu}}=0 \text { uniformly in } \frac{1}{q} \leqq R s \leqq \frac{1}{p}
$$

for any non-negative integers $j$ and $\mu$, but

$$
\lim _{\nu \rightarrow \infty}\left|\sum_{\mu=0}^{r} \alpha_{\mu} \frac{d^{\mu} \psi_{\nu}^{\left(s_{0}\right)}}{d s^{\mu}}\right|=\infty .
$$

Proof. Consider the class of all complex-valued indefinitely differentiable even functions $f(x)$ of a real variable $x$ for which

$$
\left|\frac{d^{b} f}{d x^{b}}\right| \leqq c_{a, b}\left|\frac{\exp \{(1 / 2-1 / p)|x|\}}{1+|x|^{a}}\right|
$$

for all non-negative integers $a, b$ and all real numbers $x$; here $c_{a, b}$ are constants independent of $x$. This class of functions $f(x)$ is clearly a linear space on which we define topology by the sequence of semi-norms 


$$
\sup _{-\infty<x<\infty}\left|\frac{d^{b} f}{d x^{b}}\right|\left(1+|x|^{a}\right) \exp \left\{\left(\frac{1}{p}-\frac{1}{2}\right)|x|\right\}
$$

$(a, b=0,1,2, \cdots)$. We thus obtain a locally convex, complete topological vector space. Its image under the classical Fourier transform $f(x)$ $\rightarrow \int f(x) e^{-(8-1 / 2) x} d x=F(s)$ is precisely the space $\hat{S}_{00}^{p}$ defined above (and this mapping is a topological isomorphism). If $h(x)=h(-x)$ is indefinitely differentiable and of compact support and if $H(s)=\int h(x) e^{-(s-1 / 2) x} d x$ then

$$
\sum_{\mu=0}^{r} \alpha_{\mu} \frac{d^{\mu} H}{d s^{\mu}}\left(s_{0}\right)=\sum_{\mu=0}^{r} \alpha_{\mu} \int x^{\mu} e^{\left(s_{0}-1 / 2\right) x} h(x) d x .
$$

Now, $\sum_{\mu=0}^{r} \alpha_{\mu} x^{\mu} e^{\dot{(s 0}-1 / 2) x}$ does not lie in the dual of the space defined by (4.9) and (4.10) for $R s_{0}>1 / p$. Thus we can find a sequence of even indefinitely differentiable functions $f_{v}(x)$ of compact support which converge in the topology (4.10) to a function $f(x)$ in the space (4.9) but such that

$$
\lim _{\nu \rightarrow \infty}\left|\int_{-\infty}^{\infty} \sum_{\mu=0}^{r} \alpha_{\mu} x^{\mu} e^{\left(s_{0}-1 / 2\right) x} f_{\nu}(x) d x\right|=\infty .
$$

Now take $\psi_{\nu}(s)=\int f_{\nu}(x) e^{(s-1 / 2) x} d x$ and the lemma follows.

Theorem 4.1. Let $m$ and $n$ be fixed integers. Let $s_{0}$ be any complex number with $R s_{0}>1 / p$. Let $\beta_{0}, \beta_{1}, \cdots, \beta_{r}$ be given complex numbers, such that $\beta_{0}=0$ if $m \cdot n>0$ and $s_{0}$ is an integer satisfying $1<s_{0} \leqq 1+\min \{|m|,|n|\}$ or if $s_{0}$ is an integer for which $u_{m n}\left(g, s_{0}\right)=0$ for all $g \in G$ (see formulas (2.15) and (2.15a) of Part II). Then $\sum_{\mu=0}^{r} \beta_{\mu} u_{m n}^{(\mu)}\left(g, s_{0}\right)$ is not in the dual of $S_{m n}^{p}$.

Proof. It is an immediate consequence of Theorem 2.1 of $\$ 2$ of Part II that we can find a function $F(s) \in \mathfrak{T}_{0} D_{m n}$ such that

(a) If $m n>0$, then $F(s)=0$ for $s=2,3, \cdots, 1+\min (|m|,|n|)$.

(b) If $m n>0$ and $s_{0}=2,3, \cdots, 1+\min (|m|,|n|)$, or if $s_{0}$ is an integer for which $u_{m n}\left(g, s_{0}\right)=0$ identically in $G$, then $F^{(1)}\left(s_{0}\right)=1$ and $F^{(j)}\left(s_{0}\right)=0$ for $j=0,2,3, \cdots, r$.

(c) If $s_{0}$ is not an integer satisfying the conditions imposed in (b), then $F\left(s_{0}\right)=1, F^{(j)}\left(s_{0}\right)=0$ for $j=1,2, \cdots, r$.

By the above lemma, we can find a sequence of functions $\psi_{\text {, }}$ satisfying (4.7) and (4.8), where we set $\alpha_{\mu}=\beta_{\mu}$ if $s_{0}$ is not one of the exceptional integers described in the statement of Theorem 4.1, or $\alpha_{\mu}=\beta_{\mu} / r$ if $s_{0}$ is one of those integers. For each $\nu$, let us set $H_{\nu}=F \psi_{\nu}$, so $H_{\nu}$ is in $T_{0} D_{m n}$ by the above construction and Theorem 2.1 of Part I.

Denote by $h_{\nu}=\mathfrak{T}_{0}^{-1} H_{\nu}$, so $h_{\nu} \in D_{m n}$. Moreover, $\mathfrak{T} h_{\nu}=\left\{H_{\nu}(s), 0,0\right\}$ because, if $m n>0$ then $H_{\nu}(l)=0$ for $l=2,3, \cdots, 1+\min (|m|,|n|)$. Thus, by the definition of the topology of $S_{m n}^{p}$ and Theorem 3.2, $\left\{h_{\nu}\right\}$ converges in $S_{m n}^{p}$. On the other hand, 


$$
\begin{aligned}
\left|\int \sum_{\mu=0}^{r} \beta_{\mu} u_{m n}^{(\mu)}\left(g, s_{0}\right) h_{\nu}(g) d g\right| & =\left|\sum_{\mu=0}^{\mu} \beta_{\mu}\left[\frac{d^{\mu}}{d s^{\mu}} \int u_{m n}(g, s) h_{\nu}(g) d g\right]_{s=s_{0}}\right| \\
& =\left|\sum_{\mu=0}^{r} \beta_{\mu} H_{\nu}^{(\mu)}\left(s_{0}\right)\right|=\left|\sum_{\mu=0}^{r} \alpha_{\mu} \psi_{\nu}^{(\mu)}\left(s_{0}\right)\right| \rightarrow \infty
\end{aligned}
$$

as $\nu \rightarrow \infty$. Thus, $\sum_{\mu=1}^{r} \beta_{\mu} u_{m n}^{(\mu)}\left(g, s_{0}\right)$ cannot lie in the dual of $S_{m n}^{p}$ which is the desired result.

Theorem 4.2. Let $f \in E_{m n}$ be mean periodic and let $1<p \leqq 2$. If $f$ is in the dual of $S_{m n}^{p}$ then the set of complex numbers $s_{j}$ which occurs in the mean-periodic expansion (4.1) is contained in the union of the strip $1 / q \leqq R s \leqq 1 / p$ and the rational integers.

Proof. The mean periodic expansion of $f$ of $\S 5$ of Part II is of the form

$$
\sum \sum d_{j q} u_{m n}^{(q)}\left(g, s_{j}\right) \text {. }
$$

We have shown (see Lemma 4.1) that, for any $r$,

$$
\sum_{j} d_{j r} u_{m n}^{(j)}\left(g, s_{r}\right)=W_{r} * f
$$

for some $W_{r} \in E_{m m}^{\prime}$. Now, it follows from the main theorem on the Fourier transform of $S_{m n}^{p}$ that $W_{r} * S_{m n}^{p} \subset S_{m n}^{p}$. Thus, $W_{r} * f \in\left(S_{m n}^{p}\right)^{\prime}$, the dual of $S_{m n}^{p}$. But, we have already shown that $\sum_{j} d_{j r} u_{m n}^{(j)}\left(g, s_{r}\right) \in\left(S_{m n}^{p}\right)$ can occur in only two cases: if $1 / q \leqq R s_{r} \leqq 1 / p$, or if $s_{r}$ is an integer, and $j=0$, and $u_{m n}\left(g, s_{r}\right)$ belongs to the discrete series of unitary representations of $G$. This completes the proof.

5. The ideals of $\mathfrak{D}$ and $\mathscr{F}^{\prime}$. Let $\Re$ be the set of all matrices $\left(a_{m n}\right)$ which are rapidly decreasing in $m$ and $n$, i.e. satisfy $a_{m n}=O\left(|m|^{-p} \cdot|n|^{-q}\right)$ for all positive $p, q$. Here $m$ and $n$ vary either over all integers or over all integers $\geqq$ some fixed $l$ (or $\leqq$ some fixed $l$ ). We topologize $\Re$ by saying that any polynomial $Q(m, n)$ and any $\epsilon>0$ define an open neighborhood of $\left(a_{m n}\right)$ consisting of all $\left(x_{m n}\right) \in R$ with $\left|Q(m, n) \cdot\left(x_{m n}-a_{m n}\right)\right|<\epsilon$. The open sets of $\Re$ are to be the unions of these open neighborhoods. With ordinary matrix-multiplication as product, $R$ is easily seen to be a locally convex topological algebra with the continuous involution $\left(a_{m n}\right) \rightarrow\left(a_{m n}\right)^{*}=\bar{a}_{n m}$. If $m$ and $n$ vary over all integers, then $\Re$ is clearly topologically isomorphic to the algebra of all indefinitely differentiable functions on the 2-dimensional torus with $\int_{0}^{1} a(a, y) b(y, z) d y$ as product, as follows at once using Fourier series in two real variables (see for example Schwartz [4]). Let $\mathfrak{B}$ be the topological vector space of all rapidly decreasing sequences with the topology defined by all polynomials $Q(m)$ in one variable. The elements of $\Re$ can be considered as continuous endomorphisms of $\mathfrak{B}$ by putting for $\left(x_{n}\right) \in \mathfrak{B},\left(a_{m n}\right): x_{m} \rightarrow \sum_{n} a_{m n} x_{n}$. Clearly this is simultaneously continuous in $\left(a_{m n}\right) \in \Re$ and $\left(x_{n}\right) \in \mathfrak{B}$. 
We shall now study the ideals of the group algebras $D$ and $E^{\prime}$. We first note

Lemma 5.1. The mapping $J \rightarrow J \cap D$ is a 1-1 correspondence between all closed left- (right-, two-sided) ideals $J$ of $E^{\prime}$ and all closed left- (right-, two-sided) ideals of $D$.

Proof. Let $J$ be any closed left ideal of $E^{\prime}$. Since $D * E^{\prime} \subseteq D$, we have $D * J \subseteq D \cap J \subseteq J$. Therefore for their closures in $E^{\prime}$ we have $\mathrm{Cl}(D * J) \subseteq \mathrm{Cl}(D \cap J)$ $\subseteq J$. For any $W \in E^{\prime}$ there exist elements $f_{j} \in D$ such that $W=\lim f_{j} * W$; hence $\operatorname{Cl}(D * J)=J$ and so also $\operatorname{Cl}(D \cap J)=J$. This proves that the mapping $J \rightarrow D \cap J$ is one-one. Moreover since the topology of $D$ is stronger than that induced by $E^{\prime}, D \cap J$ is a closed left-ideal of $D$. It remains to prove that every closed left-ideal $\Im$ of $D$ is of the form $D \cap J$ where $J$ is a closed left-ideal of $E^{\prime}$. Let $J$ be the closure $\bar{\Im}$ of $\Im$ in $E^{\prime}, J=\bar{\Im}$. We have to prove that $\bar{\Im} \cap D=\Im$. Let $W \in \bar{\Im}$, then there are elements $f_{\alpha} \in \Im$ such that $f_{\alpha} \rightarrow W$ in the topology of $E^{\prime}$. Hence by the continuity of convolution, $h * f_{\alpha} \rightarrow h * W$ in the topology of $D$, thus $D * \bar{\Im} \subseteq \Im$. On the other hand $D * I$ is dense in $I$ in the topology of $D$. But $D * \bar{\Im} \subseteq D \cap \bar{\Im}$. Also $D * \Im$ is dense in $D \cap \bar{\Im}$ in the topology, because for any $f \in D \cap \bar{\Im}$ there are elements $f_{j} \in D$ with $f_{j} \rightarrow f$ in the topology of $E^{\prime}$, hence again by the continuity of convolution $h * f_{j} \rightarrow h * f$ in the topology of $D$. This proves that $D \cap \bar{\Im}=\Im$. This completes the proof of Lemma 5.1 for left-ideals. The same argument applies to closed right-ideals, hence also to closed twosided ideals.

Lemma 5.2. Let $J_{s}$ be the set of all those $f \in D$ for which $F_{m n}(s)=0$ for all $m$ and $n$, for this given s. Then $J_{s}$ is a proper closed two-sided ideal of $D . J_{s}$ is self-adjoint if and only if $R s=1 / 2$, or $s$ is a real number which is not an integer.

Proof. We know that for each fixed $s$ the mapping $f \rightarrow F(s)=\left[F_{m n}(s)\right]$ is a homomorphism of $D$ into the algebra $R$ of all rapidly decreasing matrices introduced at the beginning of this paragraph. This homomorphism is continuous by the corollary to Theorem 3.3 of Part II. Hence $J_{s}$ is a closed twosided ideal of $D$.

The assertion about the self-adjointness of $J_{s}$ is proved as follows. Suppose that $J_{s}^{*}=J_{s}$. Then $\left(P_{00} J_{s}\right)^{*}=P_{00} J_{s}^{*}=P_{00} J_{s}$. Hence the elementary spherical function $\phi(g, s)=u_{00}(g, s)$ must satisfy $\phi^{*}(g, s)=\phi(g, s)$ for this $s$. It follows from Theorem 1 and Theorem 8 of Part I that this holds if and only if $R s=1 / 2$ or $s$ is real.

If $R s=1 / 2$, then $U(g, s)$ is unitary, hence $J_{s}$ is clearly self-adjoint. Now let $s$ be real and $f(g) \in J_{s}$, i.e.

$$
\int f(g) u_{m n}(g, s) d g=F_{m n}(s)=0
$$

for this $s$ and all $m, n$. Then 


$$
\begin{aligned}
\int f^{*}(g) u_{m n}(g, s) d g=\int f\left(g^{-1}\right) u_{m n}(g, s) d g \\
=\overline{\int f\left(g^{-1}\right) \bar{u}_{m n}(g, s) d g}=\overline{\int f(g) \bar{u}_{m n}\left(g^{-1}, s\right) d g} \\
=\overline{\int f(g) u_{n m}(g, 1-\bar{s}) d g}=\overline{\int f(g) u_{n m}(g, 1-s) d g} \\
=\chi_{n m}(s) \int f(g) u_{n m}(g, s) d g=\chi_{m n}(s) F_{n m}(s) .
\end{aligned}
$$

If $s \neq 0, \pm 1, \pm 2, \cdots$, then $\chi_{n m}(s) \neq 0$ for all $n, m$ (see equation (3.5) of $\S 3$ of Part I or $\S 1$ above); hence $F_{m n}(s)=0$ if and only if $\chi_{n m}(s) F_{n m}(s)=0$ for all $m, n$. This proves that if $s$ is any real number not an integer, then $f \in J_{s}$ if and only if $f^{*} \in J_{s}$, i.e. $J_{s}^{*}=J_{s}$.

Now let $s$ be any rational integer. It follows from Theorem 2.1 of Part II that we can find integers $m, n$ such that

$$
\left(\mathfrak{T}_{0} D_{m n}\right)(S)=0, \text { but }\left(\mathfrak{I}_{0} D_{n m}\right)(S) \neq 0 .
$$

For this $m, n$ the function $\chi_{m n}$ has a pole of order 1 at $s$. Let $f \in D_{m n}$ such that $\mathfrak{T}_{0} f$ has a zero of order 1 at $s$; by Theorem 2.1 of Part II such $f$ exist. Then the above shows that $F_{n m}^{*}=\chi_{m n} F_{m n}$ is different from zero at $s$. Thus $f \in J_{s}$, but $f^{*} \in J_{s}$. This completes the proof of Lemma 5.2.

REMARK 1. If $s$ is an integer, then by Theorem 5.1 below, there exist three closed maximal two-sided ideals of $D$ corresponding to $s$. By a similar reasoning one shows that each of these three ideals is self-adjoint.

Lemma 5.3. Let $J$ be a closed two-sided ideal of $D$. Then for any $h \in J$ and any $m, n=0, \pm 1, \pm 2, \cdots$, we have $P_{m n} h \in J$.

Proof. It is easily seen that

$$
P_{m n} h=\left(P_{m m} \delta\right) * h *\left(P_{n n} \delta\right)
$$

where $\delta$ is the delta-measure of $1 \in G$. Now we may write $\delta=\lim f^{j}$ in the topology of $E^{\prime}$, where $\left\{f^{j}\right\}$ is a sequence of elements of $D$. The assertion of Lemma 5.3 now follows from the continuity of the projections $P_{i i}$ (see Lemma 2.1 of Part II) the continuity of convolution in the topology of $D$ and the fact that $J$ is closed in $D$.

Lemma 5.4. Let $J$ be a maximal two-sided closed ideal in $D$. For each $m, n$, set $J_{m n}=J \cap D_{m n}$. Then each $J_{m n}$ is either a maximal closed left module over $D_{m m}$ or is equal to $D_{m n}$.

Proof. Assume there exists a proper closed left module $\widetilde{\Im}$ in $D_{m n}$ over $D_{m m}$ such that $\widetilde{\Im} \supset J_{m n}, \widetilde{\Im} \neq J_{m n}$. Let $\Im$ be the closed two-sided ideal in $D$ 
generated by $\tilde{\Im} \cup J$, and set $I_{m n}=\Im \cap D_{m n}$. We claim that $\Im_{m n}=\widetilde{\Im}$. For, if $h \in \tilde{\Im} \cup J$ and $f \in D$, then by Lemma 2.5 of Part II and the continuity of convolution,

$$
P_{m n}(f * h)=\sum_{j} f_{m j} * h_{j n}
$$

where the series converges in $D$. Now, for $j \neq m, h_{j n} \in J$ by Lemma 5.4. So, since $J$ is an ideal, $f_{m j} * h_{j n} \in J_{m n} \subset \widetilde{\Im}$. On the other hand, $h_{m n} \in \widetilde{\Im}$. So, since $\widetilde{\Im}$ is a left module over $D_{m n}, f_{m m} * h_{m n} \in \widetilde{\Im}$. Thus, for all $j, f_{m j} * h_{j n} \in \widetilde{\Im} ;$ since $\widetilde{\Im}$ is closed, this means that $P_{m n}(f * h) \in \widetilde{\Im}$.

By the results of $\S 5$, of Part II, $\widetilde{\Im}$ is also a closed right module over $D_{n n}$. Thus, by reasoning exactly as above, we see that $P_{m n}(h * f) \in \widetilde{\Im}$.

Thus, $\Im$ is a proper closed ideal which clearly contains $J$. This is impossible since $J$ was assumed to be maximal.

We shall now find all maximal closed two-sided ideals of $D$. Let $J$ be a maximal closed proper two-sided ideal; for each $m, n$, denote by $J_{m n}=J \cap D_{m n}$, so by Lemma 5.4, $J_{m n}$ is either a maximal left module over $D_{m m}$ or else $J_{m n}$ $=D_{m n}$. Since $J \neq D$, for some $p, q$ we must have $J_{p q} \neq D_{p q}$, so $J_{p q}$ is a maximum left module over $D_{m m}$. Thus, by the corollary to Theorem 5.1 of Part II there is some complex number $s_{0}$ such that, if $s_{0}$ is not an integer, $J_{p q}$ consists of all $F(s) \in T_{0} D_{p q}$ for which $F\left(s_{0}\right)=0$, which if $s_{0}$ is an integer, either $J_{p q}$ consists of all $F(s)$ in $T_{0} D_{p q}$ for which $F\left(s_{0}\right)=0$, or else $J_{p q}$ consists of all $F(s) \in T_{0} D_{p q}$ with $F^{\prime}\left(s_{0}\right)=0$ (depending on the values of $p$ and $q$ ). There are now two possibilities:

CASE 1. $s_{0}$ is not an integer. We claim that, for any $m, n, J_{m n}$ consists of all $H(s) \in T_{0} D_{m n}$ which satisfy $H\left(s_{0}\right)=0$. For, assume first there is an $H(s)$ $\in J_{m n}$ with $H\left(s_{0}\right) \neq 0$.

Let us set (see $\$ 2$ of Part II) for $|n| \geqq|m|$

$$
\eta_{m n}(s)=\prod_{j=1}^{|n|}(j-s) \prod_{j=1}^{|m|}(j-1+s)
$$

and define $\eta_{m n}(s)$ for $|m| \geqq|n|$ by

$$
\eta_{m n}(s)=\prod_{j=1}^{|m|}(j-s) \prod_{j=1}^{|n|}(j-1+s) .
$$

By Theorem 4.1 of Part II we have $\eta_{m n}(s) \in T_{0} E_{m n}^{\prime}$, so we may write

$$
\eta_{m n}(s)=T_{0} \epsilon_{m n}
$$

where $\epsilon_{m n} \in E_{m n}^{\prime}$. Then we know that $\eta_{p m}(s) H(s) \eta_{n q}(s) \in J_{p q}$ because $J$ is a closed two-sided ideal in $D$, hence also a closed two-sided module over $E^{\prime}$ (because $D$ is dense in $E^{\prime}$, and because of the continuity of convolution), and $\eta_{p m} \in T_{0} E_{p m}, \eta_{n q} \in T_{0} E_{p m}$. But, $\eta_{p m}\left(s_{0}\right) \neq 0, \eta_{n q}\left(s_{0}\right) \neq 0$, so that $\eta_{p m}\left(s_{0}\right) H\left(s_{0}\right) \eta_{n q}\left(s_{0}\right)$ $\neq 0$. This contradicts the fact that all functions of $J_{p q}$ vanish at $s_{0}$. We con- 
clude that every $H(s) \in J_{m n}$ must vanish at $s_{0}$. But, the set of functions of $T_{0} D_{m n}$ which vanish at $s_{0}$ is a maximum left module over $T_{0} D_{m m}$. Thus, $J_{m n}$ consists of all functions of $T_{0} D_{m n}$ which vanish at $s_{0}$.

We have shown that if there is a maximal two-sided ideal $I$ in $D$ corresponding to the point $s_{0} \neq 0, \pm 1, \pm 2, \cdots$ then $\Im$ must consist of all $\mathfrak{F}(s) \in T_{0} D$ such that $F_{m n}\left(s_{0}\right)=0$ for all $m, n$. It is clear that $\Im$ is actually a two-sided proper closed ideal, and the above argument shows that $\Im$ is maximal.

CASE 2. $s_{0}$ is an integer. Let us assume first that $s_{0}=-l$ is a negative integer or zero. Then we examine the following diagram which represents the way the algebra $T_{0} D$ breaks up at $s_{0}$ :

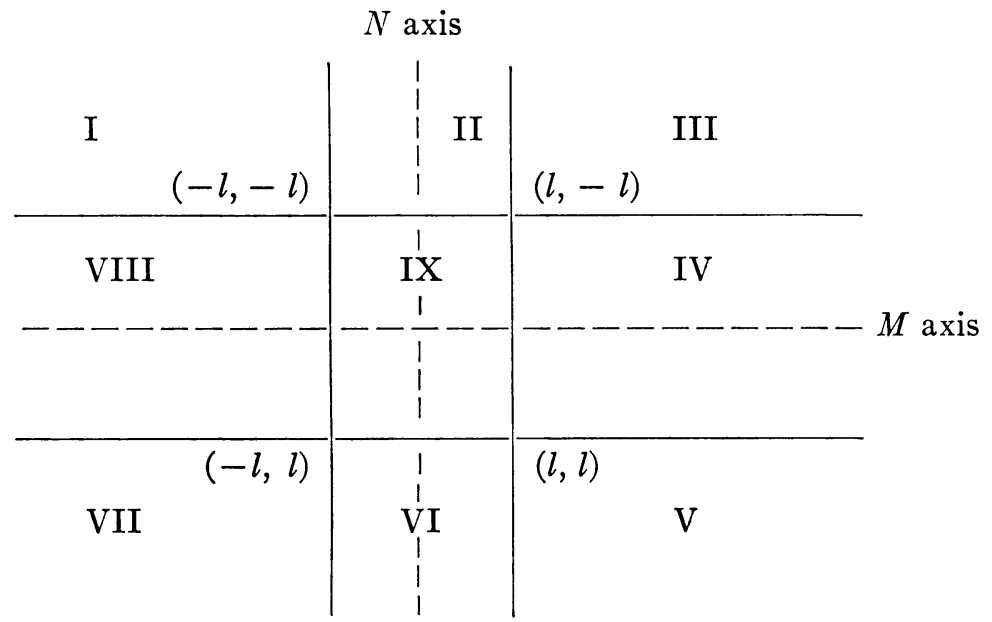

It follows from Theorem 2.1 of Part II that for $(m, n)$ in III, IV, VII, or VIII, we have $\left(T_{0} D_{m n}\right)\left(s_{0}\right)=0$.

We must now consider the nine possible places where $(p, q)$ may lie:

PlACE I. Here $J_{p q}$ consists of all $F(s) \in T_{0} D_{p q}$ which satisfy $F\left(s_{0}\right)=0$. We claim that, for any other $(m, n)$ in $I, J_{m n}$ consists of all functions $H(s) \in T_{0} D_{m n}$ with $H\left(s_{0}\right)=0$. For, assume first that there is an $H(s) \in J_{m n}$ satisfying $H\left(s_{0}\right)$ $\neq 0$. Then, as in Case 1 above, we have $\eta_{p m}(s) H(s) \eta_{n q}(s) \in J_{p q}$. But, $\eta_{p m}\left(s_{0}\right)$ $\neq 0, \eta_{n q}\left(s_{0}\right) \neq 0$, which implies $\eta_{p m}\left(s_{0}\right) H\left(s_{0}\right) \eta_{m q}\left(s_{0}\right) \neq 0$, which is a contradiction. Thus, every $H(s) \in J_{m n}$ must vanish at $s_{0}$ and, as in Case $1, J_{m n}$ consists of all $H(s) \in T_{0} D_{m n}$ which vanish at $s_{0}$. Again as in Case 1, we find that the ideal $J^{1}$ consisting of all $h \in D$ with $H_{m n}\left(s_{0}\right)=0$ for $m, n \in I$ is a maximal two sided proper closed ideal.

Place V. By the same reasoning we obtain the ideal $J^{2}$ consisting of all $h \in D$ for which $H_{m n}\left(s_{0}\right)=0$ if $m, n \in$ Place IX.

PLACE IX. By the same reasoning as above, we obtain the maximal ideal $J^{3}(m, n)$ in IX, consisting of all $h \in D$ for which $H_{m n}\left(s_{0}\right)=0$ if $m n \in \mathrm{IX}$.

Place II. We deduce as above that for any $m, n \in I$, every $H(s) \in T_{0} D_{m n}$ 
must vanish at $s_{0}$. Thus, $J \subset J^{1}$ and so is not maximal.

PLACE III. Here $J_{p q}$ consists of all $H \in T_{0} D_{p q}$ with $H^{\prime}\left(s_{0}\right)=0$. As above, we find that for any $m n \in$ Place I every $F \in J_{m n}$ must vanish at $s_{0}$. Thus, $J \subset J^{1}$ and thus is not maximal.

Place IV. Here again, $J_{p q}$ consists of all $H \in T_{0} D_{p q}$ with $H^{\prime}\left(s_{0}\right)=0$. We find that $J \subset J^{3}$ and so is not maximal.

Place VI. Similar to Place II.

Place VII. Similar to Place III.

Place VIII. Similar to Place IV.

Putting all our results together, we see that, if $J$ is a maximal ideal corresponding to the point $s_{0}=-l$, then $J=J^{1}$ or $J^{2}$ or $J^{3}$.

On the other hand, if $s_{0}=l+1$ is a positive integer, then $\left(T_{0} D_{m n}\right)\left(s_{0}\right)=0$ in the places II, III, VI, and VII. We find as before, three maximal ideals: $I^{1}=$ set of all $\mathfrak{F}(s) \in \mathfrak{T}_{0} D$ such that $F_{m n}(l+1)=0$ for $(m, n)$ in Place I, $I^{2}=$ set of all $\mathfrak{F}(s) \in \mathfrak{T}_{0} D$ for which $F_{m n}(l+1)=0$ for $(m, n)$ in Place $\mathrm{V}$ and $I^{3}=$ set of $\mathfrak{F}(s) \in \mathfrak{T}_{0} D$ with $F_{m n}(l+1)=0$ for $m, n$ in place IX. In virtue of the functional equation for $\mathfrak{F}(s), I^{1}=J^{1}, I^{2}=J^{2}$, and $I^{3}=J^{3}$.

Summing up our results, we have:

THEOREM 5.1. Every complex number $s_{0}$ which is not a rational integer determines one proper closed two-sided maximal ideal of $D$, namely, the set $J_{s_{0}}$ of all $f \in D$ for which $T_{0} f=\mathfrak{F}(s) \in \mathfrak{I}_{0} D$ satisfies $F_{m n}\left(s_{0}\right)=0$ for all $m$ and $n$. Every integer $s_{0}=0, \pm 1, \pm 2, \cdots$ determines three proper closed two-sided maximal ideals: (1). The set $J_{s_{0}}^{1}$ of all $\mathfrak{F}(s) \in \mathfrak{T}_{0} D$ such that $F_{m n}\left(s_{0}\right)=0$ for $(m, n)$ in Place I. (2) The set $J_{s_{0}}^{2}$ of all $\mathfrak{F}(s) \in \mathfrak{T}_{0} D$ for which $F_{m n}\left(s_{0}\right)=0$ for $(m, n)$ in Place V. (3) The set $J_{s_{0}}^{3}$ of all $\mathfrak{F}(s) \in \mathfrak{I}_{0} D$ for which $F_{m n}\left(s_{0}\right)=0$ for $m, n$ in Place IX (where $s_{0}=-l$ if $s_{0}$ is a negative integer or zero, or $s_{0}=l+1$ if $s_{0}$ is a positive integer). We have $J_{s_{0}}=J_{1-s_{0}}$ if $s_{0}$ is not an integer, and $J_{s_{0}}^{1}=J_{1-s_{0}}^{1}$, $J_{s_{0}}^{2}=J_{1-s_{0}}^{2}$ and $J_{s_{0}}^{3}=J_{1-s_{0}}^{3}$ if $s_{0}$ is an integer, and these are the only relations among the maximal ideals described above. Mortover, the ideals $J_{s_{0}}, J_{s_{0}}^{1}, J_{s_{0}}^{2}$, and $J_{s_{0}}^{3}$ are all the proper closed two-sided maximal ideals of $D$.

Using Theorem 5.1 and a well-known property of entire functions of exponential type we deduce

CoROllary 5.1. Let $\left\{s_{r}\right\}$ be any sequence of complex numbers which are not rational integers such that the number of $s_{r}$ with absolute value $\leqq Q$ is not $O(Q)$. Then $\bigcap_{r} J_{s_{r}}=0$. In particular, the intersection of all the maximal proper closed two-sided ideals of $D$ consists of 0 only.

Now, let $J$ be a closed two-sided ideal of $D$; we have shown above that for each $m, n, P_{m n} J=J_{m n} \subset J$. Let $s_{0}$ be any complex number which is not an integer and suppose that $T_{0} J_{p q}$ has a zero of order $r$ at $s_{0}$. From the relations $\epsilon_{m p} * J_{p q} * \epsilon_{q n} \subset J_{m n}$ and $\epsilon_{p m} * J_{m n} * \epsilon_{n q} \subset J_{p q}$ (where $\epsilon_{i j}$ is defined in (5.2) above) and the fact that $\left(T_{0} \epsilon_{i j}\right)\left(s_{0}\right) \neq 0$ (see 5.1 above) we deduce that $T_{0} J_{m n}$ has a zero of order $r$ at $s_{0}$ for any $m, n$. 
By similar reasoning we deduce that if $s_{0}$ is an integer and $T_{0} J_{p q}$ has a zero of order $r$ as $s_{0}$, where $p, q$ lies in Place I, IV, or IX (see the proof of Theorem 5.1 above) then for any $m, n$ which is in the same Place as $p, q$, then $T_{0} J_{m n}$ has a zero of order $r$ at $s_{0}$.

If $s_{0}$ is an integer and $(p, q),(m, n)$ are any pairs of integers, then the above reasoning shows that the order of zero of $T_{0} J_{p q}$ at $s_{0}$ can differ from the order of zero of $T_{0} J_{m n}$ at $s_{0}$ by at most 2 , because the functions $\eta_{m n}(s)$ are polynomials with simple zeros.

This leads us to the following

Definition 5.1. Let $J$ be a two-sided closed ideal in $D$. Then the spectrum of $J$ consists of

(a) The pairs $\left(s_{0}, r\right)$ where $s_{0}$ is a complex number, not a rational integer and for any $p, q T_{0} J_{p q}$ has a zero of order $r \geqq 0$ at $s_{0}$.

(b) The pairs $\left(s_{0}, r^{1}\right),\left(s_{0}, r^{2}\right)$ and $\left(s_{0}, r^{3}\right)$ where $s_{0}$ is an integer and $T_{0} J_{p q}$ has a zero of order $r^{1}$ at $s_{0}$ for $(p, q)$ in Place I, $T_{0} J_{p q}$ has a zero of order $r^{2}$ for $(p, q)$ in Place IV, and $T_{0} J_{p q}$ has a zero of order $r^{3}$ for $(p, q)$ in Place IX.

(If a function is not zero at $s_{0}$, we say the function has a zero of order $-\infty$ at $s_{0}$; thus it is possible that some of the $r^{j}$ are $-\infty$.)

(c) The pairs $\left(s_{0}, r_{p q}\right)$ where $s_{0}$ is an integer, $p q$ does not belong to any of the Places I, IV, or IX, and $T_{0} J_{p q}$ has a zero of order $r_{p q}$ at $s_{0}$.

Theorem 5.2. Every closed two-sided ideal in $D$ is determined by its spectrum.

Proof. Let $J$ be a closed two-sided ideal in $D$; then we know by Lemma $\mathbf{5 . 3}$ that $P_{m n} J \subseteq J$ for all $m$ and $n$. It follows from Lemma 2.4 of Part II that $J$ is determined by the $P_{m n} J$ for all $m, n$. By Theorem 5.2, each $P_{m n} J$ is determined by its spectrum; our above remarks show that the spectrum of $P_{m n} J$ is determined by the spectrum of $J$. This completes the proof of Theorem 5.2.

REMARK. Because of Lemma 5.1 the results of Theorems 5.1 and 5.2 apply to $E^{\prime}$ in place of $D$.

6. The spectrum of a two-sided mean-periodic function. In $\S 6$ of Part II we studied the two-sided closed submodules of $E$ and two-sided meanperiodic functions. We shall now obtain further properties of them and show in particular that every two-sided mean-periodic function has a spectrum.

LeMma 6.1. Let $M$ be a closed two-sided submodule of $E$. Let $M^{\perp \perp}$ denote the set of all $w \in E^{\prime}$ such that $w * M=M * w=(0)$. Then $\left(M_{m n}\right)^{\perp}=\left(M^{\perp \perp}\right)_{m n}$, where $\left(M_{m n}\right)^{\perp}$ is defined to be the set of those $v \in E_{m n}^{\prime}$ which satisfy $v\left(M_{m n}\right)=(0)$.

Proof. Let $w \in M^{\perp \perp}$ and put $w_{m n}=P_{m n} w$. Then we have $w * f=f * w=0$ for any $f \in M$. Since $M$ is a closed two-sided $G$-submodule of $E$ (hence also a module over $E^{\prime}$ ) we know that $P_{m n} M=M_{m n} \subseteq M$. Hence $w * f=0$ for any $f \in M_{m n}$, hence $w(f)=0$. But $w(f)=w_{m n}(f)$. This proves that $\left(M^{\perp \perp}\right)_{m n} \subseteq\left(M_{m n}\right)^{\perp}$. Conversely, let $v \in\left(M_{m n}\right)^{\perp}$, i.e. $v \in E_{m n}^{\prime}$ and $v(f)=0$ for all $f \in M_{m n}$. Then $v(f)$ $=0$ for all $f \in M$, because $v \in E_{m n}^{\prime}$ implies $v\left(E_{p q}\right)=0$ for $(p, q) \neq(m, n)$ and 
$M_{m n} \subseteq M$. But $M$ is a two-sided $G$-module, hence $v\left(L_{g} f\right)=v\left(R_{\theta} f\right)=0$ for all $g \in G$ and $f \in M$. It follows that $v * f=f * v=0$ for any $f \in M$.

In exactly the same way one proves

LeMmA 6.2. Let $J$ be a closed two-sided ideal of $E^{\prime}$. Let $J^{\text {LL }}$ denote the set of all $f \in M$ which satisfy $f * J=J * f=(0)$. Then $\left(J^{\perp \perp}\right)_{m n}=\left(J_{m n}\right)^{\perp}$, where $\left(J_{m n}\right)^{\perp}$ is defined to be the set of all those $f \in E_{m n}$ which satisfy $w(f)=0$ for all $w \in J_{m n}$.

Lemma 6.3. $\left(M^{\perp \perp}\right)^{\perp \perp}=M$ and $\left(J^{\perp \perp}\right)^{\perp \perp}=J$.

Proof. Clearly $M \subseteq\left(M^{\perp \perp}\right)^{\perp \perp}$. Now assume $h \in\left(M^{\perp \perp}\right)^{\perp \perp}$, but $h \notin M$. By the Hahn-Banach theorem there exists an element $w \in E^{\prime}$ such that $w(h)=1$ and $w(M)=(0)$. Then $w * M=M * w=(0)$, thus $w \in M^{\perp \perp}$. But $w * h \neq 0$, which contradicts $h \in\left(M^{\perp \perp}\right)^{\perp \perp}$. This proves the lemma for any closed two-sided $G$-module $M$ of $E$. Similarly one proves $\left(J^{\perp \perp}\right)^{\perp \perp}=J$.

Lemma 6.4. Let $V$ be a closed submodule of $E_{m n}$ (say a left-module over $E_{m m}^{\prime}$, see $\S 5$ of Part II) and $V^{\perp}$ the set of elements $w \in E_{m n}^{\prime}$ such that $w(V)=(0)$. Let $M$ be the closed two-sided G-module generated by $V$ in $E$ and $J$ the closed twosided ideal generated by $V^{\perp}$ in $E^{\prime}$. Then the spectra of $J$ and $M^{\perp \perp}$ (as defined in $\$ 5$ above) are equal except when $s_{r}=0, \pm 1, \pm 2, \cdots$ in which case the orders of zeros of $J$ at $s_{r}$ may be two larger than those of $M^{\perp}$.

Proof. Denoting again by $u_{m n}^{(j)}\left(g, s_{r}\right)$ the $j$ th derivative of $u_{m n}(g, s)$ with respect to $s$ at $s_{r}$ (for fixed $g$ ) we know by Theorem 5.2 of $\$ 5$ of Part II that $V$ is the smallest closed submodule of $E_{m n}$ (over $E_{m m}^{\prime}$ say) which contains all those $u_{m n}^{(j)}\left(g, s_{r}\right)$ which are contained in $V$ (and not identically zero in $g$ ).

Now for any $g, a \in G$

$$
\sum_{l} u_{m l}(g, s) u_{l n}(a, s)=u_{m n}(g a, s)
$$

where the series on the left converges for fixed $m, n$ in the topology of $E$ uniformly in $s$ for $s$ in any compact subset of the $s$-plane. This follows from Lemma 1.2 of Part II and an argument as that in the proof of Lemma 3.1 of $\S 3$ of Part II by applying the group invariant Laplacian $\Delta$ and (left- and right-) derivatives in the direction of $K$ to the above equation. It follows that we can differentiate termwise with respect to $s$ since each term in the above series $\sum_{l}$ is entire in $s$. So we obtain

$$
u_{m n}^{(j)}(g a, s)=\sum_{i=0}^{j}\left(\begin{array}{l}
j \\
i
\end{array}\right) \sum_{l=-\infty}^{\infty} u_{m l}^{(i)}(g, s) u_{l n}^{(j-i)}(a, s)
$$

with the series converging in the topology of $E$ uniformly in $s$ for $s$ in a compact set. It now follows that $u_{m n}^{(j)}\left(g, s_{r}\right) \in V$ implies $u_{p q}^{(i)}\left(g, s_{r}\right) \in M$ for any $i \leqq j$ and all $p, q$, by applying the projections $P_{\alpha \beta}$ to (6.1) and using induction. Also the finite linear combinations $\sum_{i, r, p, q} c_{i r p q} u_{p q}^{(i)}\left(g, s_{r}\right)$ are dense in $M$ where $c_{i r p q}$ are arbitrary complex numbers, as follows from (6.1) and the definition of $M$. 
Now we apply $P_{p q}$ and we see that $M_{p q}=P_{p q} M$ is the closure of the finite linear combination $\sum_{i, r}^{p} c_{i r} u_{p q}^{(i)}\left(g, s_{r}\right)$ for fixed $p, q$ where $s_{r}$ varies again exactly over those complex numbers for which $u_{m n}^{(j)}\left(g, s_{r}\right) \in V$ and $0 \leqq i \leqq j$ (and for which $u_{m n}^{(j)}\left(g, s_{r}\right)$ is not identically zero in $\left.g\right)$.

In order to complete the proof of Lemma 6.4, it is sufficient to show (by Lemma 6.1) that for any $p$ and $q, T_{0} J_{p q}$ consists exactly of those functions in $T_{0} E_{p q}^{\prime}$ which vanish at $s_{r}$ to the order $j_{r}$, except for some possible exceptions if $s_{r}$ is an integer (see Theorem 5.2 above). This follows immediately from the fact that, for any $p$ and $q$,

$$
T_{0} J_{p q}=\eta_{p m}(s)\left(T_{0} J_{m n}\right) \eta_{n q}(s) .
$$

Equation (6.2) can be proved as follows: $J$ is, by definition, the closure in $E^{\prime}$ of the set of linear combinations of $W * V^{\perp} * W^{\prime}$ for $W, W^{\prime} \in E^{\prime}$. Applying $P_{m n}$ which is a continuous projection of $E^{\prime}$ onto $E_{m n}^{\prime}$ by Lemma 4.5 of Part II, we see that $J_{m n}$ is the closure of the set of linear combinations of $Z * V^{\perp} * Z^{\prime}$ for $Z \in E_{p m}^{\prime}, Z^{\prime} \in E_{n q}^{\prime}$. Equation (6.2) now results from the above and the fact that, for any $H(s) \in T_{0} E_{p m}^{\prime}$, we can write $H(s)=\eta_{p m}(s) F(s)$ where $F(s)$ $=F(1-s)$, with a similar expression for the functions in $T_{0} E_{n q}^{\prime}$.

Theorem 6.1. Suppose that for each $m$ and $n$ we are given a closed submodule $V_{m n}$ of $E_{m n}$ with the property that the closed two-sided $G$-module $M$ in $E$ generated by all $V_{m n}$ is different from $E$. For each $m, n$, denote by $V_{m n}^{\perp}$ the set of all $w \in E_{m n}^{\prime}$ with $W\left(V_{m n}\right)=0$; call ${ }_{m n} J$ the closed ideal in $E^{\prime}$ generated by $V_{m n}^{\perp}$ and set $J=\bigcup_{m n} J$. Then $M^{\perp \perp} \supseteq J$. Moreover, the spectrum of $M^{\perp \perp}$ is the same as that of $J$ except that, if $s_{r}$ is an integer, some of the orders of variety of $J$ may be two greater than the corresponding orders of $M^{\perp \perp}$.

Proof. For each $m, n$ call ${ }_{m n} M$ the closed two-sided $G$-module generated by $V_{m n}$. Then the theorem follows from the above Lemma 6.4 expressing the relationship between $\left({ }_{m n} M\right)^{\perp \perp}$ and ${ }_{m n} J$, and the fact that $M$ is the closure of the set of linear combinations of the ${ }_{m n} M$ so that $M^{\perp \perp}$ is the intersection of the $\left({ }_{m n} M\right)^{\perp \perp}$.

Definition. Let $f \in E$ be two-sided mean-periodic. Consider the twosided module $M_{f}=M$ generated by $f$ in $E$ (over $G$ ). Let $J=M^{\perp \perp}$. We call the spectrum of $J$ (as defined in $\$ 5$ above) the spectrum of $f$.

Now consider $f_{m n}=P_{m n} f$. By $\S 5$ and $\S 6$ of Part II $f_{m n}$ is mean-periodic in $E_{m n}$ and the spectrum of $f_{m n}$ is defined as the spectrum of $V_{m n}$ (in the sense of $\S 5$ of Part II) where $V_{m n}$ is the (left-) module (over $E_{m m}^{\prime}$ ) generated by $f_{m n}$ in $E_{m n}$. From Theorem 6.1 we obtain

Theorem 6.2. Let $f$ be two-sided mean-periodic and $f_{m n}=P_{m n} f$. If $s$ is any complex number $\neq 0, \pm 1, \pm 2, \cdots$ then $s$ is in the spectrum of $f$ if and only if $s$ is in the spectrum of $f_{m n}$ for at least one pair $m, n$. The order of the spectrum of $f$ at $s$ equals $\max _{m, n}$ of the order of the spectrum of $f_{m n}$ at $s$. If $s$ is a rational integer, then the orders of the spectrum of $f$ at $s$ are equal to one or two less 
than the $\max _{m, n}$ of the order of the spectrum of $f_{m n}$ at $s$. In particular the set of complex numbers $s_{j}$ which occurs in the mean-periodic expansion of $f$ (of $\$ 6$ of Part II) is discrete and except possibly at $s=0, \pm 1, \pm 2, \cdots$ it coincides with the set of complex numbers occurring in the spectrum of $f$ defined just above.

7. Ideals of $L^{1}(G)$. In $\S 4$ and $\S 5$ of Part I we studied the ideals of the closed commutative subalgebra $L_{00}^{1}=P_{00} L^{1}(G)$ of $L^{1}(G)$. (In Part I we wrote $A_{1}$ instead of $L_{00}^{1}$.) We shall now obtain more properties of the ideals of $L_{00}^{1}$ and consider at the same time properties of the ideals of $P_{m m} L^{1}(G)=L_{m m}^{1}$ and of $L^{1}(G)$.

Lemma 7.1, The function $u_{m n}(g, s)$ of $g$ is bounded only in the following cases:

(i) $0 \leqq R s \leqq 1$ and any fixed $m, n$.

(ii) If $m>0$ and $n>0$ for $s=1, \cdots, 1+\min (m, n)$ or $s=0, \cdots$, $-\min (m, n)$.

(iii) If $m<0$ and $n<0$ for $s=1, \cdots, 1-\max (m, n)$ or $s=0, \cdots$, $\max (m, n)$.

(iv) Those values of $m, n, s$ for which $u_{m n}(g, s)=0$ for all $g \in G$ (see (2.15) and (2.15a) of $\$ 2$ of Part II). If $m=n$ the case (iv) does not occur.

Also $u_{0 n}^{(1)}(g, 0)=\partial u_{0 n}(g, s) /\left.\partial s\right|_{s=0}$ is a bounded function of $g$.

Proof. The assertion about $u_{m n}(g, s)$ is a special case of Theorem 4.1 of $\S 4$ above with $p=1$. It remains to prove the assertion about $u_{0 n}^{(1)}(g, 0)$ $=\partial u_{0 n}(g, s) /\left.\partial s\right|_{s=0}$. We use again

$$
\begin{aligned}
& u_{0 n}(g, s)=\int_{0}^{1} \frac{d g \theta^{s}}{d \theta} e^{-2 \pi i n \theta} d \theta \\
& u_{0 n}^{(1)}(g, s)=\int_{0}^{1}\left[\frac{d g \theta}{d \theta}\right]^{s} \log \frac{d g \theta}{d \theta} e^{-2 \pi i n \theta} d \theta .
\end{aligned}
$$

Hence

$$
u_{0 n}^{(1)}(g, 0)=\int_{0}^{1} \log \frac{d g \theta}{d \theta} e^{-2 \pi i n \theta} d \theta .
$$

Now put $g=g_{\xi}$ and use that

$$
\frac{d g_{\xi} \theta}{d \theta}=\left|\cosh \xi+e^{2 \pi i \theta} \sinh \xi\right|^{-2}=(\cosh \xi)^{-2} \cdot\left|1+e^{2 \pi i \theta} \tanh \xi\right|^{-2}
$$

so that

$$
\log \frac{d g_{\xi} \theta}{d \theta}=-2 \log \cosh \xi-2 \log \left|1+e^{2 \pi i \theta} \tanh \xi\right| .
$$


Thus for $n \neq 0$ we obtain

$$
u_{0 n}^{(1)}\left(g_{\xi}, 0\right)=-2 \int_{0}^{1}\left\{\log \left|1+e^{2 \pi i \theta} \tanh \xi\right|\right\} e^{-2 \pi i n \theta} d \theta .
$$

Now $\log \left|1+e^{2 \pi i \theta} \tanh \xi\right|$ is bounded for $\xi \geqq 2$ and all $\theta$. Hence $u_{0 n}^{(1)}\left(g_{\xi}, 0\right)$ is a bounded function of $\xi$ for $\xi \geqq 2$ for $n \neq 0$. On the other hand $u_{0 n}^{(1)}(g, s)$ is a continuous function of $g$ since $u_{m n}(g, s)$ is a real analytic function simultaneously in $g$ and $s$. This proves that $u_{0 n}^{(1)}\left(g_{\xi}, 0\right)$ is a bounded function of $\xi$ for $\xi \geqq 0$. On the other hand $u_{0 n}^{(1)}(g, s)$ is a spherical function of type $0, n$, so that $u_{0 n}^{(1)}(g, 0)$ is bounded on $G$ for $n \neq 0$.

Lemma 7.2. All closed maximal ideals of the commutative normed algebra $L_{m m}^{1}$ are given as follows.

(a) For every complex number $s_{0}$ with $0 \leqq R s_{0} \leqq 1$ the set of all $f(g) \in L_{m m}^{1}$ for which $\int f(g) u_{m m}\left(g, s_{0}\right) d g=0$ is a proper maximal closed ideal of $L_{m m}^{1}$.

$\left(b_{+}\right)$For $m>0$ there is one maximal ideal of $L_{m m}^{1}$ for each $s_{0}=2, \cdots, m+1$ consisting of those $f(g) \in L_{m m}^{1}$ for which $\int f(g) v_{m m}^{+}\left(g, s_{0}\right) d g=0$.

(b_) For $m<0$ there is one maximal ideal of $L_{m m}^{1}$ for each $s_{0}=2, \cdots, 1-m$ consisting of those $f(g) \in L_{m m}^{1}$ for which $\int f(g) v_{m m}^{-}\left(g, s_{0}\right) d g=0$.

Any two of these maximal ideals of $L_{m m}^{1}$ are unequal except in the case (a) where the ideals corresponding to $s_{0}$ and $1-s_{0}$ are equal.

Proof. Let $J$ be a (closed proper) maximal ideal of $L_{m m}^{1}$. Then $L_{m m}^{1} / J$ is naturally isomorphic with the field of complex numbers; so we get a continuous homomorphism of $L_{m m}^{1}$ onto the complex numbers with $J$ as kernel. Consider the restriction of this homomorphism of $D_{m m}$; this cannot be identically zero because $D_{m m}$ is dense in $L_{m m}^{1}$. By the Corollary to Theorem 5.1 of Part II every continuous homomorphism of $D_{m m}$ to the complex numbers is of the form $f \rightarrow \int f(g) u_{m m}\left(g, s_{0}\right) d g=F_{m m}\left(s_{0}\right)$ for some $s_{0}$. By Lemma 7.1 this can be extended to a continuous homomorphism of $L_{m m}^{1}$ only $0 \leqq R s_{0} \leqq 1$ or $s_{0}$ and $m$ in the cases (ii) or (iii) of Lemma 7.1. This proves that every continuous homomorphism of $L_{m m}^{1}$ to the complex numbers is of the form $f \rightarrow \int f(g) u_{m m}\left(g, s_{0}\right) d g$ for $0 \leqq R s_{0} \leqq 1$ or $s_{0}$ and $m$ in the cases (ii) or (iii) of Lemma 7.1 above. Now by formulas (1.7)-(1.10) of Part II $v_{m m}^{+}\left(g, s_{0}\right)=u_{m m}(g, l)$ for $m>0$ and $v_{m m}^{-}(g, l)=u_{m m}(g, l)$ for $m<0$. Using the natural 1-1 correspondence between closed maximal ideals and continuous homomorphisms to the complex numbers, we see that every closed maximal ideal of $L_{m m}^{1}$ is of the form (a) or $\left(b_{ \pm}\right)$.

The assertion about the inequality of these ideals follows for example by taking the intersection $D_{m m} \cap L_{m m}^{1}$ and applying $\$ 2$ (Theorem 2.1) of Part II.

We next have

Lemma 7.3. Let $s=\sigma+i$ be any complex number. The linear operator $U(g, s)$ maps the Lebesgue-space $L^{p}(0 \leqq \theta \leqq 1 ; d \theta)$ isometrically onto itself if and only if $\sigma=1 / p$. 
Proof. By definition of $U(g, s)$ we have $[U(g, s) a](\theta)=[d g \theta / d \theta]^{s} a(g \theta)$ $=b(\theta)$ say. Thus

$$
\int_{0}^{1}|b(\theta)|^{p} d \theta=\int_{0}^{1}\left[\frac{d g \theta}{d \theta}\right]^{\sigma p}|a(g \theta)|^{p} d \theta=\int_{0}^{1}\left[\frac{d g \theta}{d \theta}\right]^{\sigma p-1}|a(g \theta)|^{p} d g \theta
$$

is equal to $\int_{0}^{1}|a(\theta)|^{p} d \theta$ for all $a(\theta) \in L^{p}(0 \leqq \theta \leqq 1 ; d \theta)$ if and only if $\sigma p-1=0$. This proves the isometry; the fact that $U(g, s)$ is onto, follows if one notes that for $\sigma=1 / p$ the operator $U\left(g^{-1}, s\right)$ is everywhere defined on $L^{p}(0 \leqq \theta \leqq 1$; $d \theta)$ and the inverse of $U(g, s)$.

From this we deduce

Lemma 7.4. Let $f(g) \in L^{1}(G)$ and $0 \leqq \sigma \leqq 1$. Then $\int f(g) U(g, s) d g$ is a bounded operator of the Banach space $L^{p}(0 \leqq \theta \leqq 1 ; d \theta)$ for $p=1 / \sigma$, with bound $\leqq \int|f(g)| d g$.

Proof. Since $0 \leqq \sigma \leqq 1,1 \leqq p \leqq \infty$, so $L^{p}$ is a Banach space. Hence we may apply the standard theory of operator-valued integrals on Banach spaces and conclude that Lemma 7.4 is correct.

Next

LEMma 7.5. Let $s$ be any complex number in the strip $0 \leqq \sigma \leqq 1$. Let $J_{8}$ be the set of those elements $f(g) \in L^{1}(G)$ for which $\int f(g) U(g, s) d g=0$ in the sense of operator-valued integrals on $L^{p}(0 \leqq \theta \leqq 1 ; d \theta) ; p=\sigma^{-1}$. Then $J_{s}$ is a closed twosided proper ideal of $L^{1}(G)$.

Proof. The fact that $J_{s}$ is a closed two-sided ideal of $L^{1}(G)$ follows from Lemma 7.4 since the mapping $f \rightarrow \int f(g) U(g, s) d g$ is easily seen to be a homomorphism of $L^{1}(G)$ into the algebra of bounded operators of the Banach space $L^{p}(0 \leqq \theta \leqq 1 ; d \theta)$ (using the representation property of $U(g, s)$ ). The continuity of this homomorphism follows from Lemma 7.4, namely from that assertion that the bound of $\int f(g) U(g, s) d g$ is $\leqq \int|f(g)| d g$.

To prove that $J_{s}$ is proper we use Theorems 4 or 8 of Part I and conclude that there exists an $f(g) \in L_{00}^{1}$ such that $F_{00}(s) \neq 0$ for this value of $s$ (with $0 \leqq R s \leqq 1)$. It follows that $\int f(g) U(g, s)^{\dot{d}} d g \neq 0$ for this $s$. This proves that $J_{s} \neq L^{1}(G)$. Again, by Theorems 4 or 8 of Part I, we can find an $h(g) \in L_{00}^{1}$ such that $\int h(g) u_{00}(g, s) d g=H_{00}(s)$ vanishes at a given value of $s$ (in the strip $0 \leqq R s \leqq 1)$. It follows that $\int h(g) U(g, s) d g=0$ for such $h(g)$. Hence $J_{s} \neq(0)$. This proves Lemma 7.5.

REMARK. For each fixed $s$ the ideal $J_{s}$ is identical with the set of $f(g)$ $\in L^{1}(G)$ for which $\int f(g) u_{m n}(g, s) d g=0$ for all $m, n$ and this $s$, as is easily seen.

Lemma 7.6. Let $J$ be a closed two-sided ideal of $L^{1}(G)$. Then $P_{m n} J \subseteq J$. Hence $P_{m n} J=J \cap L_{m n}^{1}$.

Proof. Let $f \in J$. We have $\left(P_{m n} f\right)(g)=\int_{0}^{1} \int_{0}^{1} f\left(k_{\theta} g k_{\vartheta}\right) e^{-2 \pi i(m \theta+n \vartheta)} d \theta d \vartheta$. It is well known that $J$ is closed under right- and left-translates by elements of $G$. 
Moreover the double integral converges in the topology of $L^{1}(G)$. This implies Lemma 7.6.

Lemma 7.7. Let $s_{0}$ be any complex number in the strip $0 \leqq R s \leqq 1$. If $s_{0} \neq 0$ or 1 , then $J_{s_{0}}$ is maximal in the set of proper closed two-sided ideals of $L^{1}(G)$.

Proof. Suppose $h(g) \in L^{1}(G)$ but $h(g) \notin J_{s_{0}}$. Let $I$ be the closed two-sided ideal generated by $h$ and $J_{s_{0}}$. By the remark following Lemma 7.5 $H_{m n}\left(s_{0}\right)$ $=\int h(g) u_{m n}\left(g, s_{0}\right) d g \neq 0$ for some $m, n$. By Lemma 7.6 $\left(P_{m n} h\right)(g)=h_{m n}(g) \in I$. By Theorem 2.1 of $\S 2$ of Part II there exists a function $a(g) \in D_{n m}$ such that $A_{n m}\left(s_{0}\right)=\int a(g) U_{n m}\left(g, s_{0}\right) d g \neq 0$. Now consider the convolution $\left(h_{m n} * a\right)(g)$ $=b(g)$. Clearly $b(g) \in I$ and $P_{m m} b=b$, so that $b(g) \in P_{m m} I$. Also $B_{m m}\left(s_{0}\right)$ $=\int b(g) u_{m m}\left(g, s_{0}\right) d g=H_{m n}\left(s_{0}\right) A_{n m}\left(s_{0}\right) \neq 0$. On the other hand $P_{m m} J_{s_{0}} \subseteq I$ (since $\left.J_{s_{0}} \subseteq I\right)$. But $P_{m m} J_{s_{0}}$ is a closed maximal ideal of $L_{m m}^{1}$, by Lemma 7.2. Thus $P_{m m} I=L_{m m}^{1}$ for this value of $m$ because $b(g) \in P_{m m} I$ and $b(g) \notin P_{m m} J_{s_{0}}$. Hence $P_{m m}=P_{m m}(I \cap D)=\left(P_{m m} I\right) \cap D \neq P_{m m}\left(D \cap J_{s_{0}}\right)$. Therefore $D \cap J_{s_{0}} \neq D \cap I$. But by $\S 5$ above $J_{s_{0}} \cap D$ is maximal (in the set of closed proper two-sided ideals of $D)$. Therefore $I \cap D=D$, hence $I=L^{1}(G)$. This proves the maximality of $J_{s_{0}}$.

Now let $l=1,2, \cdots$ and $J_{l}^{+}$the set of all $f(g) \in L^{1}(G)$ for which

$$
\int_{G} f(g) v_{m n}^{+}(g, l) d g=0 \text { for all } m, n>l
$$

and $J_{l}^{-}$the set of those $f(g) \in L^{1}(G)$

$$
\int f(g) v_{m n}^{-}(g, l) d g=0 \text { for all } m, n<-l .
$$

By $J_{1}^{0}$ we denote the set of all $f(g) \in L^{1}(G)$ with $\int f(g) d g=0$.

Lemma 7.8. $J_{1}^{0}, J_{l}^{+}$, and $J_{l}^{-}$are maximal in the set of closed proper twosided ideals of $L^{1}(G)$.

The proof of Lemma 7.8 is similar to the proof of Lemma 7.7.

Lemma 7.9. Let $J$ be maximal in the set of proper closed two-sided ideals of $L^{1}(G)$. Then $P_{m n} J=L_{m n}^{1}$ or $P_{m l} J$ is maximal in the set of proper closed submodules of $L_{m n}^{1}$ which are left-modules over $L_{m m}^{1}$ and right-modules over $L_{n n}^{1}$.

Proof. By Lemma 7.6 $P_{m n} J \subseteq J$. Clearly $P_{m n} J$ is a closed subset of $L^{1}(G)$. Assume $P_{\mu \nu} J \neq L_{\mu \nu}^{1}$ for one pair $\mu, \nu$. Then let $h(g) \in L_{\mu \nu}^{1}$ but $h \notin P_{\mu \nu} J$. Consider the closed two-sided ideal $\tilde{J}$ generated by the $P_{m n} J$ for all pairs $m, n$ of integers and the function $h(g)$. Then $\tilde{J} \supseteq J$. Now $P_{\mu \nu} h=h \notin P_{\mu \nu} J$, hence $h \notin J$, so $J \neq \tilde{J}$. Therefore $\tilde{J}=L^{1}(G)$, hence of course $P_{m n}^{1} \tilde{J}=L_{m n}^{1}$ for all $m, n$.

Now consider $P_{\mu \nu} \tilde{J}$. It is the closure of the set of all linear combinations of convolutions $a * b * c$ where $a \in L_{\mu m}^{1}, b \in P_{m n} J, c \in L_{n \nu}$ as $m, n$ vary arbitrarily and $\alpha * h * \beta$ where $\alpha \in L_{\mu \mu}^{1}$ and $\beta \in L_{\nu \nu}^{1}$. This follows easily from the fact that 
$L^{1}=\sum_{m, m} L_{m, n}^{1}$ and the properties of convolutions of functions of type $m, n$. Now $P_{m n} J \subset J$ by Lemma 7.6, so that $a * b * c \in P_{\mu \nu} J$ for any $a \in L_{\mu m}^{\prime}, b \in P_{m n} J$, and $c \in L_{n \nu}^{\prime}$. Thus, $P_{\mu \nu} \tilde{J}$ is the closed two-sided module (left module over $L_{\mu \mu}^{\prime}$, right module over $L_{\nu \nu}^{\prime}$ ) generated by $P_{\mu \nu} J$ and $h$. In particular, if $\mu=\nu$, then $P_{\mu \mu} \tilde{J}$ is the closed ideal in $L_{\mu \mu}^{\prime}$ generated by $P_{\mu \mu} J$ and $h$. Thus, $P_{\mu \mu} J$ is maximal.

Lemma 7.10. Let $J$ be any closed proper two-sided ideal of $L^{1}(G)$. Then $P_{m m} J \neq L_{m m}^{1}$ for at least one $m$.

Proof. Suppose $P_{m m} J=L_{m m}^{1}$ for every $m=0, \pm 1, \pm 2, \cdots$. Then $J \cap D$ $\supseteq D_{m m}$ for every $m$. Now by the description of the closed two-sided ideals of $D$ obtained in $\S 5$ above, it follows that $J \supseteq D$. But since $D$ is clearly dense in $L^{1}$, we conclude that $J=L^{1}$.

From the last two lemmas we deduce at once

Lemma 7.11. Let $J$ be maximal in the set of proper closed two-sided ideals of $L^{1}(G)$. Then $P_{m m} J$ is maximal in the set of proper closed ideals of $L_{m m}^{1}$ for at least one $m$.

Lemma 7.12. Let $J$ be again maximal in the set of proper closed two-sided ideals of $L^{1}(G)$ and assume that for some $m, P_{m m} J$ is of the form given in Lemma 7.2 (i.e. $P_{m m} J$ is closed and maximal in the set of all proper ideals). If $P_{m m} J$ is in the case (a) of Lemma 7.2 and if $s_{0} \neq 0,1$ then $J$ is of the form $J_{s_{0}}$ in the sense of Lemma 7.5 above.

Proof. We claim that, for any $\mu, \nu$ we have $P_{\mu \nu} J$ consists of all $f \in L_{\mu \nu}^{\prime}$ such that $\int f(g) u_{\mu \nu}\left(g, s_{0}\right) d g=0$, i.e. $P_{\mu \nu} J=P_{\mu \nu} J_{s_{0}}$. First, $P_{\mu \nu} J \subset P_{\mu \nu} J_{s_{0}}$. For, if some function $f \in P_{\mu \nu} J$ has the property that $\int f(g) u_{\mu \nu}\left(g, s_{0}\right) d g \neq 0$ then we pick functions $h \in D_{m \mu}, h^{\prime} \in D_{\nu m}$ with $\int h(g) u_{m \mu}\left(g, s_{0}\right) d g \neq 0$ and $\int h^{\prime}(g) u_{\nu m}\left(g, s_{0}\right) d g$ $\neq 0$ (these exist by Theorem 2.1 of Part II); the function $h * f * h^{\prime}$ then belongs to $P_{m m} J$ but $\int\left(h * f * h^{\prime}\right)(g) u_{m m}\left(g, s_{0}\right) d g \neq 0$. This contradicts the fact that $P_{m m} J=P_{m m} J_{s_{0}}$.

Thus $J \subset J_{s_{0}}$. Since both $J$ and $J_{s_{0}}$ are maximal in the set of closed twosided ideals (see Lemma 7.7), we must have $J=J_{s_{0}}$.

By similar reasoning we can prove that if $P_{m m}$ is any of the cases of Lemma 7.2 then $J$ must be one of the maximal proper closed two-sided ideals of $L^{1}$ described in Lemmas 7.7 and 7.8.

Summarizing the above results we obtain:

THEOREM 7.1. Let $J$ be maximal $\left({ }^{4}\right)$ in the set of all proper closed two-sided ideals of $L^{1}=L^{1}(G)$. Then either $J$ is the ideal $J_{s}$ of Lemma 7.5 with $s \neq 0$ or 1 , or $J=J_{1}^{0}, J_{1}^{+}$or $J_{1}^{-}$as defined by (7.1) and (7.2) above.

Lеммa 7.13. The analogue of Wiener's theorem is not true for the commutative normed algebra $L_{00}^{1}$ : there exists an $f \in L_{00}^{1}$ which lies in no maximal closed ideal of $L_{00}^{1}$, but the closed ideal generated by $f$ in $L_{00}^{1}$ is not the whole of $L_{00}^{1}$.

(4) We call such an ideal maximal closed. 
Proof. Consider the function

$$
(z-1)^{2}(z+1)^{2} \exp \left(\frac{z+1}{z-1}+\frac{z-1}{z+1}\right) .
$$

It is holomorphic for $|z|<1$ and continuous in $|z| \leqq 1$. Now map the disc $|z|<1$ conformally onto the strip $0<R s<1$ by

$$
z=i \frac{1-\exp \pi i(s-1 / 2)}{1+\exp \pi i(s-1 / 2)}, \quad s-\frac{1}{2}=\frac{1}{\pi i} \log \frac{1+i z}{1-i z} .
$$

Let $H(s)$ be the image of (7.3) under the mapping (7.4) and put $F_{1}(s)$ $=\exp \left[(s-1 / 2)^{2}\right] H(s), F(s)=F_{1}(s)^{2}$. Then $F(s) \in \mathfrak{I}_{0} S_{00}^{1}$ and $F_{1}(s) \in \mathfrak{I}_{0} S_{00}^{1}$. By a result of Beurling [1, Theorem 1 ] one cannot approximate $H(s)$ uniformly in the strip $0 \leqq R s \leqq 1$ by functions $X(s) F(s)$ where $X(s)$ can vary over all functions which are holomorphic in $0<R s<1$, continuous in $0 \leqq R s \leqq 1$ and vanish $\pm i \infty$. Moreover by Theorem 7 of Part I (see Ehrenpreis and Mautner [1]) one cannot approximate $F_{1}(s)$ uniformly by functions $X(s) F(s)$ with $X(s)$ as above. Since the topology of $\mathfrak{T}_{0} L_{00}^{1}$ is stronger than uniform convergence, it follows that if we put $f=\mathfrak{T}_{0}^{-1} F(s)$, then the closed ideal generated by $f$ in $L_{00}^{1}$ is not the whole of $L_{00}^{1}$. Moreover $F(s) \neq 0$ for any $s$ in the strip $0 \leqq R s \leqq 1$, hence by Lemma $7.2 f$ does not belong to any maximal closed ideal of $L_{00}^{1}$.

THEOREM 7.2. There exists a function $h(g) \in L^{1}=L^{1}(G)$ which belongs to no maximal closed two-sided ideal of $L^{1}$, but the closed two-sided ideal generated by $h(g)$ in $L^{1}$ is not the whole of $L^{1}$. Thus the analogue of Wiener's theorem does not hold here.

Proof. Let $B$ be the matrix all of whose coefficients are zero except the $(0,0),(1,1)$ and $(-1,-1)$ coefficient which are 1 . Let $c_{l m n}$ be sufficiently decreasing positive constants and form

$$
h(g)=\mathfrak{I}_{0}^{-1}[F(s)]+\sum_{l=1}^{\infty} \sum_{m, n} c_{l m n}\left[v_{m n}^{+}(g, l)+\bar{v}_{m n}^{-}(g, l)\right] .
$$

By Theorem $3.1 \mathfrak{I}_{0}^{-1}[F(s) B] \in L^{1}$, also $v_{m n}^{ \pm}(g, l) \in L^{1}$ for all $m, n$ if $l$ $=1,2,3, \cdots$ by Bargmann [1]. Hence for sufficiently decreasing $c_{l m n}$, $\sum_{l, m, n} c_{l m n}^{+}\left[v_{m n}^{+}(g, l)+v_{m n}^{-}(g, l)\right] \in L^{1}$. Therefore $h(g) \in L^{1}$.

By Theorem $7.1 h(g)$ belongs to no maximal closed two-sided ideal of $L^{1}$. Let $J$ be the closed two-sided ideal generated by $h$ in $L^{1}$. Then from the above construction of $h(\mathrm{~g})$ we see that $P_{00} J$ is the closed ideal generated in $L_{00}^{1}$ by the function $f(g)$ of the proof of Lemma 7.13 ; hence $P_{00} J \neq L_{00}^{1}$, therefore $J$ cannot be equal to $L^{1}$.

8. Functions on $G / \Gamma$. We now wish to study the analysis of functions on the coset space $G / \Gamma$ where $\Gamma$ is a given closed subgroup of $G$. In this section 
we restrict ourselves to the case where $G / \Gamma$ is compact. In addition to the case of the subgroup of triangular matrices we have a large class of subgroups of $G$ for which $G / \Gamma$ is compact. These are the fundamental groups (with possibly fixed points) of the compact Riemann-surfaces. This is the class of subgroups with which we shall be concerned in the present section. Thus we assume throughout this section that $\Gamma$ is an arbitrary discrete subgroup of $G$ such that the coset space $G / \Gamma$ is compact.

Lemma 8.1. There exists an invariant measure in $G / \Gamma$, namely the Haarmeasure on $G$ restricted to a fundamental domain for $\Gamma$ in $G$.

Proof. Since $\Gamma$ is discrete, it is unimodular, since $G$ is semisimple it is also unimodular, hence there exists an invariant measure on $G / \Gamma$. The existence of a fundamental domain was proved under very much more general conditions by C. L. Siegel [1].

We shall need a certain Riemann metric and the corresponding LaplaceBeltrami Operator on the compact (3-dimensional real analytic) manifold $G / \Gamma$. For this purpose we remind the reader that the unique two-sided invariant indefinite Riemann-metric on $G$ is obtained as follows.

The Lie algebra of $G$ is naturally isomorphic to the Lie algebra of all real $2 \times 2$ matrices $X$ of trace 0 . The nondegenerate quadratic form trace $\left(X^{2}\right)$ is invariant under the adjoint group, hence defines (by translation) a two-sided invariant nondegenerate Riemann metric on $G$. Since $G$ is a noncompact simple Lie group, this Riemann metric is not definite. This can also be seen directly by putting

$$
X_{0}=\left(\begin{array}{rr}
0 & 1 \\
-1 & 0
\end{array}\right), \quad X_{1}=\left(\begin{array}{rr}
1 & 0 \\
0 & -1
\end{array}\right), \quad X_{2}=\left(\begin{array}{ll}
0 & 1 \\
1 & 0
\end{array}\right)
$$

and $X=\sum_{0}^{2} x_{j} X_{j}$. Then trace $\left(X^{2}\right)=2\left(-x_{0}^{2}+x_{1}^{2}+x_{2}^{2}\right)$.

For any element $a$ in the Lie algebra and any differentiable function $f(g)$ we put again

$$
\left(L_{a} f\right)(g)=\lim _{t \rightarrow 0} t^{-1}\left[f\left(e^{t a} g\right)-f(g)\right]
$$

and

$$
\left(R_{a} f\right)(g)=\lim _{t \rightarrow 0} t^{-1}\left[f\left(g e^{t a}\right)-f(g)\right]
$$

Letting $a_{j}$ correspond to $X_{j}$, the Laplace-Beltrami operator corresponding to the fundamental bilinear form (or invariant Riemann metric) $2^{-1}$ trace $\left(X^{2}\right)$ $=-x_{0}^{2}+x_{1}^{2}+x_{2}^{2}$ is

$$
\Delta=-L_{a_{0}}^{2}+L_{a_{1}}^{2}+L_{a_{2}}^{2}=-R_{a_{0}}^{2}+R_{a_{1}}^{2}+R_{a_{2}}^{2} .
$$


This is (up to a consant multiple) the same as the Laplace-Beltrami operator $\Delta$ on $G$ which we used in Parts I and II. We note that $\Delta$ is not elliptic, but that $\Delta+2 L_{a_{0}}^{2}$ and $\Delta+2 R_{a_{0}}^{2}$ are elliptic operators, in fact they are the LaplaceBeltrami operators corresponding to the one-sided invariant Riemann metrics on $G$ which are obtained from $x_{0}^{2}+x_{1}^{2}+x_{2}^{2}$ by one-sided translations on $G$. However, whereas $\Delta$ commutes with both left- and right-translations on $G$, $\Delta+2 L_{a_{0}}^{2}$ (respectively $\Delta+2 R_{a_{0}}^{2}$ ) commutes with right-translations (respectively left-translations).

Now we go over to the compact manifold $G / \Gamma$. We identify the functions on $G / \Gamma$ with functions $f(g)$ on $G$ satisfying $f(g \gamma)=f(g)$ for $g \in G$ and $\gamma \in \Gamma$. The right invariant Riemann-metric on $G$ obtained from $x_{0}^{2}+x_{1}^{2}+x_{2}^{2}$ defines a positive definite nondegenerate Riemann metric on $G / \Gamma$ and the corresponding Laplacian is

$$
\Delta_{1}=\Delta+2 L_{a_{0}}^{2} \text { applied to functions } f(g)=f(g \gamma) .
$$

Let $L_{2}(G / \Gamma)$ be the Hilbert space of equivalence classes of complex valued square integrable functions on $G / \Gamma$ relative to the invariant measure on $G / \Gamma$. Applying well known results to the elliptic operator $\Delta_{1}$, we see that there exists a set of real-analytic eigenfunctions $\psi$ of $\Delta_{1}$ which is complete and orthonormal in $L_{2}(G / \Gamma)$. If $\Delta_{1} \psi=\tilde{\lambda} \psi$, then $\Delta_{1}[L(k) \psi]=L(k) \Delta_{1} \psi=\tilde{\lambda} L(k) \psi$ where $L(k)$ denotes as usual left-translation by the element $k$ of $K$. This is true because $\Delta_{1} L_{a_{0}}=L_{a_{0}} \Delta_{1}$, whence $\Delta_{1} L(k)=L(k) \Delta_{1}$ since $a_{0}$ is an element of the Lie algebra corresponding to the one-parameter subgroup $K$ of rotations in $G$. Hence

$$
\Delta_{1} \int_{0}^{2 \pi} e^{i n \theta} L\left(k_{\theta}\right) \psi d \theta / 2 \pi=\bar{\lambda} \int_{0}^{2 \pi} e^{i n \theta} L\left(k_{\theta}\right) \psi d \theta / 2 \pi .
$$

Therefore for fixed $\tilde{\lambda}_{j}$ we can introduce in the space of eigenfunctions of $\Delta_{1}$ a basis which is reduced under $K$, say $\psi_{j, 1}, \psi_{j, 2}, \cdots, \psi_{j, d_{j}}$. Then

$$
\Delta \psi_{j, r}=\Delta_{1} \psi_{j, r}+2 L_{a_{0}}^{2} \psi_{j, r}=\left[\tilde{\lambda}_{j}+2(i n)^{2}\right] \psi_{j, r}=\lambda \psi_{j, r}
$$

where

$$
\lambda_{j}=\tilde{\lambda}_{j}+2(i n)^{2}=\tilde{\lambda}_{j}-2 n^{2} .
$$

Thus $\psi_{j, r}$ is also an eigenfunction of $\Delta$. Thus we obtain a complete orthonormal set of eigenfunctions in $L_{2}(G / \Gamma)$ also for the operator $\Delta$. But since $\Delta$ commutes with left-translation $L(g)$ for every $g \in G$ we obtain a discrete decomposition of $L_{2}(G / \Gamma)$ into irreducible invariant subspaces $H_{j}$ under the unitary representation $g \rightarrow L(g)$ of $G$ :

$$
L_{2}(G / \Gamma)=\sum \oplus H_{j}
$$

LEMMA 8.2. Under the unitary representation $g \rightarrow L(g)$ of $G$, the Hilbert- 
space $L_{2}(G / \Gamma)$ decomposes into a discrete direct sum (8.4) of irreducible invariant subspaces $H_{j}$. In the space $H_{j}$ there exists a complete orthonormal set of real analytic functions $\psi_{j, n}$ such that

$$
\begin{aligned}
\Delta \psi_{j, n} & =\lambda_{j} \psi_{j, n}, \\
L_{a_{0}} \psi_{j, n} & =i n \psi_{j, n}, \\
\Delta_{1} \psi_{j, n} & =\left(\lambda_{j}+2 n^{2}\right) \psi_{j, n} .
\end{aligned}
$$

Here either $n=0, \pm 1, \pm 2, \pm 3, \cdots$ or $n=-l,-(l+1), \cdots$ or $n=l, l+1$, $l+2, \cdots$.

We have already proved all assertions of Lemma 8.2 except those about the range of $n$ and that the eigenvalue of $\psi_{j, n}$ under $L_{a_{0}}$ is exactly in. But these facts follow from the results of Bargmann [1, §5, especially p. 609] noting that $H_{j}$ is an irreducible unitary representation space of $G$.

Since $G / \Gamma$ is a compact real analytic manifold and $\Delta_{1}$ the Laplace-Beltrami operator with respect to a positive definite (real-analytic) Riemann metric on $G / \Gamma$, we have the following well known result: There exists for a suitable complex number $c$ a power $r$ of $\left(\Delta_{1}+c I\right)^{-1}$ which is an integral operator, i.e.

$$
\left[\left(\Delta_{1}+c I\right)^{-r} \phi\right](x)=\int_{G / \mathbf{r}} \mathcal{K}(x, y) \phi(y) d y
$$

for any $\phi \in L_{2}(G / \Gamma)$ and $\Re(x, y)$ is such that $\int_{G / \Gamma}|\Re(x, y)|^{2} d y$ exists, is continuous in $x$ and hence is $\leqq$ constant independent of $x \in G / \Gamma$.

Applying this to our above eigen-functions $\psi_{j, n}$ we obtain

$$
\left[\left(\Delta_{1}+c I\right)^{-r} \psi_{j, n}\right](x)=\left[2 n^{2}+\lambda_{j}+c\right]^{-r} \psi_{j, n}(x)=\int_{G / \Gamma} \mathcal{K}(x, y) \psi_{j, n}(y) d y .
$$

Applying Schwarz's inequality, we get

$$
\begin{aligned}
\left|\psi_{j, n}(x)\right| & \leqq\left|2 n^{2}+\lambda_{j}+c\right| r\left(\int|\mathcal{K}(x, y)|^{2} d y\right){ }^{1 / 2}\left\|\psi_{j, n}\right\| \\
& =O\left(\left[2 n^{2}+\lambda_{j}\right]^{r}\right) \text { uniformly in } x \in G / \Gamma \text { as }|n| \rightarrow \infty \text { and }\left|\lambda_{j}\right| \rightarrow \infty .
\end{aligned}
$$

This proves

LEMma 8.3. Let $\psi_{j, n}(x)$ be the complete orthonormal set of eigen-functions in $L_{2}(G / \Gamma)$ of the operator $\Delta_{1}$ introduced above. There exists a real number $r$ such that as $2 n^{2}+\lambda_{j} \rightarrow \infty$

$$
\psi_{j, n}(x)=O\left(\left[2 n^{2}+\lambda_{j}\right]^{r}\right)
$$

uniformly in $x \in G / \Gamma$.

REMARK. The smallest possible value of $r$ is not known. In this section it is sufficient to know that $r$ exists and is finite. 
The $\psi_{j, n}(g)$ can clearly be multiplied by arbitrary constants of absolute value one. After multiplying the $\psi_{j, n}$ by suitable such constants we have

THEOREM 8.1. The following infinite series converge uniformly in $g$ for $g$ in any compact subset of $G$, and uniformly in $x$ :

$$
\psi_{j, m}(g x)=\sum_{n} u_{n m}\left(g, s_{j}\right) \psi_{j, n}(x) .
$$

Here $s_{j}$ is such that $\lambda_{j}=$ const. $s_{j}\left(1-s_{j}\right)$ and

$$
u_{m n}\left(g, s_{j}\right)=\left\{\begin{array}{l}
u_{m n}\left(g, s_{j}\right) \text { if } R s_{j}=1 / 2, \\
\downarrow_{m}(s) \bar{\downarrow}_{n}(s) u_{m n}(g, s) \text { if } 1 / 2<s=\text { real }<1, \\
v_{m n}^{ \pm}\left(g, l_{j}\right) \text { if } s_{j} \text { is an integer } \geqq 1 .
\end{array}\right.
$$

Here the $\downarrow_{m}(s)$ are a sequence of suitable normalizing functions which occur in Bargmann's [1, pp. 618-619] construction of the exceptional series of irreducible unitary representations of $G$, so that the $\downarrow_{m}(s) \bar{\iota}_{n}(s) u_{m n}(g, s)$ are for $1 / 2<s=$ real $<1$ the normalized matrix coefficients of the irreducible representations in the exceptional series of Bargmann $[1, \S 8]$.

Proof. The space $H_{j}$ is an irreducible unitary representation space of $G$, the $\psi_{j, n}$ form a complete orthonormal sequence in $H_{j}$ and are eigenvectors under $K$. It follows from the results of Bargmann [1] that the multiplicity of each $K$-eigenspace is one-dimensional. Hence after multiplying each $\psi_{j, n}$ by a suitable constant of absolute value one, it follows that the matrix coefficients of the unitary representation of $G$ in the space $H_{j}$ with respect to the $\psi_{j, n}$ must be those explicitly computed by Bargmann [1], i.e. formula (8.7) above.

To prove the uniform convegence of (8.6) we know by Lemma 8.3 that $\left|\psi_{j, n}(x)\right|$ is for fixed $j$ smaller than a certain power of $|n|$ uniformly in $x$. Now by Lemma 1.2 of Part II $u_{n m}(g, s)$ is, for fixed $m$ and $s, O\left(|n|^{q}\right)$ for every $q$, uniformly in $g$ for $g$ in any compact set. Similarly for $v_{n m}^{ \pm}(g, l)$ by Lemma 1.3 of Part II. Moreover by expression (8.10) on p. 619 of Bargmann [1] we have $\left|\iota_{m}(s)\right| \leqq 1$. Thus for fixed $m$ and $s$ we have $u_{n m}\left(g, s_{j}\right)=O\left(|n|^{a}\right)$ for every $q$ uniformly in $g$ for $g$ in any compact subset of $G$. Hence the infinite series in (8.6) converges uniformly in $g$ for $g$ in any compact subset of $G$, and uniformly in $x$.

To prove the equality in (8.6) we note that $\sum_{n} u_{n m}\left(g, s_{j}\right) \psi_{j, n}(x)$ converges for fixed $g$ to $\psi_{j, m}(g x)$ in the $L_{2}(G / \Gamma)$-norm. But we have just seen that the infinite series converges uniformly in $x$. This proves the equality and completes the proof of Theorem 8.1.

We shall now consider the space $D(G / \Gamma)$ of all indefinitely differentiable complex valued functions on the compact real analytic manifold $G / \Gamma$. We use on $D(G / \Gamma)$ the topology of L. Schwartz.

THEOREM 8.2. Let $f \in D(G / \Gamma)$ then 


$$
a_{j, n}=\int_{G / \mathbf{\Gamma}} f(g) \bar{\psi}_{j, n}(g) d \dot{g}
$$

satisfies

$$
a_{j, n}=O\left[\left(1+\left|\lambda_{j}\right|+|n|\right)^{-p}\right]
$$

for every $p \geqq 0$. Conversely given complex numbers $a_{j, n}$ satisfying (8.9) then they are the expansion coefficients of some $f(g) \in D(G / \Gamma)$ in the sense of (8.8). Moreover the series

$$
\sum a_{j, n} \psi_{j, n}(g)
$$

converges to this function $f(g)$ in the topology of $D(G / \Gamma)$.

On the linear space of double sequences $a_{j, n}$ satisfying (8.9) we define a topology by means of the semi-norms (one for every $p=0,1,2, \cdots$ )

$$
\sup _{j, n}\left(1+\left|\lambda_{j}\right|+|n|\right)^{p}\left|a_{j, n}\right| \text {. }
$$

Then the mapping (8.8) $f(g) \rightarrow a_{j, n}$ is a topological isomorphism of $D(G / \Gamma)$ onto this topological vector space of double sequences $a_{j, n}$.

Proof. From (8.5) and (8.8) we get for every $p=0,1,2, \cdots$

$$
\left(\lambda_{j}+i n\right)^{p} a_{j, n}=\int_{G / \Gamma}\left(\Delta-L_{a_{0}}\right)^{p} f(g) \bar{\psi}_{j, n}(g) d \dot{g}
$$

as $\Delta$ is self-adjoint and $L_{a_{0}}$ skew-adjoint. Since $f(g)$ is an indefinitely differentiable function on the compact manifold $G / \Gamma,\left(\Delta+L_{a_{0}}\right)^{p} f(g)$ is a bounded continuous function. Hence $\left(\lambda_{j}+i n\right)^{p} a_{j, n}=O(1)$ for every $p=0,1,2, \cdots$. This proves (8.9).

Now consider any series $\sum a_{j, n} \psi_{j, n}(g)$ where the coefficients $a_{j, n}$ satisfy (8.9). By Lemma $8.3 \psi_{j, n}(g)=O\left(\left[\left|\lambda_{j}\right|+n^{2}\right] r\right)$ for some $r$. Now the number of eigenvalues $\leqq N$ of the Laplace-Beltrami operator $\Delta_{1}$ acting on the compact manifold $G / \Gamma$ is majorized by a power of $N$ (see Minakshisundaram and Pleijel [1]). Hence the series $\Delta_{1}^{p} \sum a_{j, n} \psi_{j, n}$ converges uniformly on $G / \Gamma$ for every $p \geqq 0$. Now $\Delta_{1}$ is elliptic, hence its powers define the topology of $D(G / \Gamma)$. Thus $\sum a_{j, n} \psi_{j, n}$ converges in the topology of $D(G / \Gamma)$. The series converges of course also in the $L_{2}(G / \Gamma)$-norm, hence $a_{j, n}$ are the Fourier-coefficients of $\sum a_{j, n} \psi_{j, n}$.

The fact that the mapping $f(g) \rightarrow a_{j, n}$ is a topological isomorphism follows now easily by the same kind of argument (cf. the analogous proof for Fourier series in L. Schwartz [4, vol. II]).

Corollary. There exists no group element $g$ for which $\psi_{j, n}(g)=0$ for all $j$ and $n$.

Proof. If $\psi_{j, n}\left(g_{0}\right)=0$ for all $j$ and $n$ then the series (8.10) would be 0 for 
$g=g_{0}$, thus every $f \in D(G / \Gamma)$ would vanish at $g=g_{0}$, which is impossible.

By $D^{\prime}(G / \Gamma)$ we denote the space of distributions on the compact manifold $G / \Gamma$ in the sense of $L$. Schwartz, i.e. the dual space of $D(G / \Gamma)$ with the strong topology for dual spaces.

In analogy to Theorem 8.2 we have

Theorem 8.3. Let $W \in D^{\prime}(G / \Gamma)$, then

$$
a_{j, n}=W \cdot \psi_{j, n}
$$

satisfies

$$
a_{j, n}=O\left(\left[1+\left|\lambda_{j}\right|+|n|\right]^{p}\right)
$$

for some $p$. Conversely given complex numbers $a_{j, n}$ satisfying (8.12), then the series $\sum a_{j, n} \psi_{j, n}$ converges in the strong topology of $D^{\prime}(G / \Gamma)$ and $a_{j, n}$ are the Fourier coefficients of the distribution $\sum a_{j, n} \psi_{j, n}$ in the sense of (8.11).

The proof is an easy analogue of the proof of Theorem 8.2.

Now let $C_{j}$ be the orthogonal projection of the Hilbert space $L_{2}(G / \Gamma)$ onto the closed subspace $H_{j}$. We denote the restriction of $C_{j}$ to the space $D(G / \Gamma)$ of indefinitely differentiable functions on $G / \Gamma$ again by $C_{j}$.

Lemma 8.4. $C_{j}$ is a continuous open mapping of $D(G / \Gamma)$ in the topology of $D(G / \Gamma)$.

Proof. This is an immediate consequence of Theorem 8.2 , because by Theorem 8.2 we need only prove the corresponding statement for the space of double sequences $a_{j, n}$. But for this space the continuity of the projection mapping follows immediately from the definition of the topology.

Now let $\delta_{\Gamma}$ be the Dirac-delta measure of the point $\Gamma \in G / \Gamma$, i.e. if $f \in D(G / \Gamma)$ we put

$$
\delta \mathrm{r} f=f(1)=f(\gamma) \text { for any } \gamma \in \Gamma
$$

where $f(g)=f(g \gamma)$. We also put

$$
\delta_{j} f=\delta \Gamma\left(C_{j} f\right) .
$$

Since $\delta_{\Gamma}$ is clearly a distribution on the manifold $G / \Gamma$, i.e. $\delta_{\Gamma} \in D^{\prime}(G / \Gamma)$, Lemma 7.4 implies at once that $\delta_{j} \in D^{\prime}(G / \Gamma)$; moreover since $H_{j}$ is invariant under $L(g)$ for each $g \in G$, we have $C_{j} L(g)=L(g) C_{i j}$ and clearly $\left[L(\gamma) \delta_{\Gamma}\right] f$ $=\delta_{\Gamma} f$. Hence for any $f \in D^{\prime}(G / \Gamma)$

$$
\begin{aligned}
{\left[L(\gamma) \delta_{j}\right] f } & =\delta_{j}[L(\gamma) f]=\delta_{\Gamma}\left\{C_{j}[L(\gamma) f]\right\} \\
& =\delta \Gamma\left\{L(\gamma) C_{j} f\right\}=\delta_{\Gamma}\left(C_{j} f\right)=\delta_{j} f
\end{aligned}
$$

This proves

Lemma 8.5. Equation (8.14) defines a distribution $\delta_{j}$ on $G / \Gamma$, [i.e. $\delta_{j}$ 
$\left.\in D^{\prime}(G / \Gamma)\right]$ satisfying

$$
L(\gamma) \delta_{j}=\delta_{j} \text { for every } \gamma \in \Gamma .
$$

Note that $\delta_{j} \neq 0$ if $H_{j} \neq(0)$ because $L(g) \psi_{j, n} \in H_{j} \cap D(G / \Gamma)$ and $\delta_{j}\left(L(g) \psi_{j, n}\right)$ $=\psi_{j, n}\left(g^{-1}\right) \neq 0$ for some $n$ and $g$, if for this $j$ we have $H_{j} \neq(0)$.

Now consider the Hilbert space $H_{j}$ for fixed $j$. If $f \in H_{j}$, then $f=\sum_{n} a_{n} \psi_{j, n}$ and the $\psi_{j, n}$ are uniquely determined (up to constant multiples of absolute value 1 ) by the requirement that they be normalized eigenfunctions under the subgroup $K$. The space $H_{j} \cap D(G / \Gamma)$ is thus isomorphic to the vector space $V$ of all rapidly decreasing sequences $a_{n}$, the isomorphism is given by $f \leftrightarrow a_{n}$ as follows from Theorem 8.2 applied to a fixed $j$. We note that for $f \in H_{j} \cap D(G / \Gamma)$

$$
\delta_{j}(f)=\delta_{j}\left(\sum_{n} a_{n} \psi_{j, n}\right)=\sum a_{n} \psi_{j, n}(1) .
$$

By Lemma 8.3, $\psi_{j, n}(1)=O\left(|n|^{2 r}\right)$ thus the sequence $\psi_{j, n}(1)$ is for fixed $j$ an element of $V^{\prime}$, invariant under each $\gamma \in \Gamma$.

We now proceed to prove the converse of this. Given any irreducible unitary representation of $G$ with representation space $H$. We can ignore the constant representation for which our theorem is trivial. Then $H$ is a Hilbert space and according to Bargmann [1] there exists a complete orthonormal set $e_{n}$ in $H$ uniquely determined (up to constant multiples of absolute value 1 ) by the requirement that they be eigenvectors under the subgroup $K$ of rotations. Thus once the (maximal compact) subgroup $K$ is chosen fixed, the subspace of $H$ which consists of all $\sum a_{n} e_{n}$, with $a_{n}=O\left(n^{-p}\right)$ for every positive $p$, is intrinsically defined. We denote it by $H^{0}$ and note that $H^{0}$ is naturally isomorphic to the vectorspace $\mathfrak{B}$ of rapidly decreasing sequences defined in $\$ 6$. We use on $H^{0}$ the topology of $\mathfrak{B}$, hence the dual $\left(H^{0}\right)^{\prime}$ of $H^{0}$ is naturally isomorphic to the space $\mathfrak{B}^{\prime}$ of slowly increasing sequences.

It is easily verified that $H^{0}$ is invariant under $G$, hence $H^{0}$ and $\left(H^{0}\right)^{\prime}$ are representation spaces for $G$. Also if $y \in H^{0}$ or $\left(H^{0}\right)^{\prime}$ and $g \in G$ then $g \cdot y$ is simultaneously continuous in both variables, as is readily verified.

Now assume there exists an element $\Lambda \in\left(H^{0}\right)^{\prime}$ and $\gamma \Lambda=\Lambda$ for all $\gamma \in \Gamma$. For any element $x$ of $H^{0}$ consider the mapping

$$
x \rightarrow f_{x}(g)=\Lambda\left(g^{-1} x\right)
$$

which associates with every $x \in H^{0}$ a function $f(g)=f_{x}(g)$ such that $f(g \gamma)$ $=f(g)$ since $\gamma \Lambda=\Lambda$ for $\gamma \in \Gamma$. Now it follows from Lemmas 1.3 and 1.4 of Part II that the representation of $G$ in the topological vector space $H^{0}$ is weakly continuous, i.e. $\Lambda(g x)$ is for any fixed $x \in H^{0}$ and $\Lambda \in\left(H^{0}\right)^{\prime}$ a continuous function of $G$. Hence $f_{x}(g)$ is for each $x \in H^{0}$ a continuous function of $g$, therefore contained in $L^{2}(G / \Gamma)$. Thus (8.16) maps $H^{0}$ linearly into $L^{2}(G / \Gamma)$. Also if $x_{\alpha} \rightarrow x$ in the topology of $H^{0}$ then $f_{x_{\alpha}} f(g) f_{x}(g)$ uniformly on compact sub- 
sets of $G$ (again by Lemmas 1.3 and 1.4 of Part II). Hence by choosing a fundamental domain for $G / \Gamma$ in $G$ we see that $x_{\alpha} \rightarrow x$ in $H^{0}$ implies $f_{x_{\alpha}} \rightarrow f_{x}$ in the $L^{2}(G / \Gamma)$-norm. This proves

Lemma 8.6. The mapping (8.16) is continuous and linear from $H^{0}$ into $L^{2}(G / \Gamma)$.

Moreover we have

$$
f_{g x}=L(g) f_{x}
$$

because $f_{o x}\left(g^{\prime}\right)=\Lambda\left(g^{\prime} g^{-1} x\right)=\left[L(g) f_{x}\right]\left(g^{\prime}\right)$. Now let us denote by $\Xi_{n}$ the image of $e_{n}$ under the mapping (8.16). Then (8.17) implies at once that $\Xi_{n}$ is an eigenvector under $L(k)$ since $e_{n}$ is. Also if $m \neq n$ the $\Xi_{m}$ and $\Xi_{n}$ belong to different eigenvalues under $K$ since $e_{m}$ and $e_{n}$ do. So $\boldsymbol{\Xi}_{m}$ and $\boldsymbol{\Xi}_{n}$ are orthogonal in $L^{2}(G / \Gamma)$ for $m \neq n$ and $\Xi_{n} \neq 0$ whenever $e_{n} \neq 0$.

Combining this with Lemma 8.6 we conclude that if

$$
x=\sum a_{n} e_{n} \in H^{0} \text { then } f_{x}(g)=\sum a_{n} \Xi_{n}(g)
$$

where $\sum a_{n} \Xi_{n}$ converges in the $L^{2}(G / \Gamma)$-norm and $f_{x} \neq 0$ if $x \neq 0$. Thus the mapping (8.16) is one-one.

Now $H$ is a unitary irreducible representation space of $G$ (by hypothesis) so that

$$
g e_{m}=\sum_{n} u_{m n}(g) e_{n}
$$

where the $u_{m n}(g)$ are the matrix-coefficients of one of the (well-known) irreducible unitary representations of $G$. Now apply the mapping (8.16) to (8.19) and observe that the series in (8.19) converges in the topology of $H^{0}$ (again by Lemmas 1.3 and 1.4 of Part II). Hence by Lemma 8.6 and formula (8.17) above we obtain

$$
L(g) \Xi_{m}=\sum_{n} u_{m n}(g) \Xi_{n}
$$

where the right side now converges in the $L^{2}(G / \Gamma)$-norm for each $g \in G$. Now for the inner product $\langle,\rangle_{H}$ in the Hilbert space $H$ we have $\left\langle g e_{m}, e_{m}\right\rangle_{H}=u_{m m}(g)$ while for the inner product $\langle,\rangle_{G / \Gamma}$ in $L^{2}(G / \Gamma)$ we have

$$
\left\langle L(g) \Xi_{m}, \Xi_{m}\right\rangle_{G / \Gamma}=\left\langle\sum_{l} u_{m l}(g) \Xi_{l}, \Xi_{m}\right\rangle_{G / \Gamma}=u_{m m}(g)\left\|\Xi_{m}\right\|_{G / \Gamma}^{2}
$$

Hence for each $m$ (for which $e_{m} \neq 0$ ) the diagonal matrix coefficients differ only by a multiplicative constant (independent of $g$ ). It follows from well known properties of the irreducible unitary representations of $G$ (for instance from their explicit determination by Bargmann [1]) that we obtain unitary equivalence. We have thus proved 
Lemma 8.7. Consider the closure in $L^{2}(G / \Gamma)$ of the image of $H^{0}$ under (8.16). (This is clearly an invariant subspace under $L(g)$ for each $g \in G$.) The restriction of $L(g)$ to this subspace gives a unitary representation of $G$ which is unitarily equivalent to the given irreducible unitary representation with space $H$.

Now let $\Lambda^{1}, \Lambda^{2}, \ldots$ be any finite number of elements of $\left(H^{0}\right)^{\prime}$ and assume each $\Lambda^{i}$ is invariant under each $\gamma \in \Gamma$. For each $\Lambda^{i}$ we obtain by Lemma 8.7 one unitary mapping of the form (8.16) of $H$ into $L^{2}(G / \Gamma)$; denote the image of $H$ in $L^{2}(G / \Gamma)$ by $H_{i}$. For each $i$ we obtain functions $\Xi_{m}^{i}(g) \in H_{i}$ as the images of the $e_{m} \in H$. Put $\Lambda_{n}^{i}=\Lambda^{i}\left(e_{n}\right)$. Then $\Lambda_{n}^{i}=O\left(n^{\nu}\right)$ for some $\nu\left(\Lambda^{i}\right.$ being an element of $\left.\left(H^{0}\right)^{\prime}\right)$. Also $u_{m n}(g)$ is for fixed $m$ and $g$ rapidly decreasing in $n$ (Lemmas 1.3 and 1.4 of Part II). It follows that $\sum u_{m n}(g) \Lambda_{n}^{t}$ converges. Also by definition of $\Xi_{m}^{i}(g)=\Lambda^{i}\left(g^{-1} e_{m}\right)$; hence putting $g=1$ we get $\Xi_{m}^{i}(1)=\Lambda_{m}^{i}$. We obtain as above

$$
\Xi_{m}^{i}(g)=\sum u_{m n}(g) \Lambda_{n}^{i}
$$

Now if there are constants $e_{i}$ such that $\sum c_{i} \Lambda^{i}=0$ in $\left(H^{0}\right)^{\prime}$ then $\sum c_{i} \Lambda_{n}^{k}=0$ for each $n$, hence $\sum c_{i} \Xi_{m}^{i}(g)=0$ for each $m$ and $g$ and conversely. This proves that the multiplicity with which the given irreducible unitary representation of $G$ occurs in the decomposition of $L^{2}(G / \Gamma)$ is equal to the dimension (over the complex numbers) of the subspace of $\Gamma$-invariant elements of $\left(H^{0}\right)^{\prime}$. This completes the proof of the following theorem.

THEOREM 8.4 (Analogue of the Frobenius reciprocity theorem). Given any irreducible (weakly measurable) unitary representation $U$ of $G$ with Hilbert space $H$. Let the $e_{m}$ form a complete orthonormal sequence of eigenvectors under $K$. Let $H^{0}$ denote the linear subspace of all $\sum a_{n} e_{n}$ with $a_{n}=O\left(n^{p}\right)$ for every $p$. Put on $H^{0}$ the topology of $\mathfrak{B}$ (of $\$ 5$ above) and let $\left(H^{0}\right)^{\prime}$ be the dual space of $H^{0}$. Let $\left(H^{0}\right)_{\Gamma}^{\prime}$ be the (linear space) of those elements $\Lambda$ of $\left(H^{0}\right)^{\prime}$ which satisfy $\gamma \Lambda=\Lambda$ for every $\gamma \in \Gamma$.

Assertion. The multiplicity with which $U$ occurs (up to unitary equivalence) in the decomposition of $L^{2}(G / \Gamma)$ is equal to the dimension of $\left(H^{0}\right)_{\Gamma}^{\prime}$.

REMARK. If the given irreducible representation $U$ of $G$ is not the trivial (constant) representation it is necessary to introduce a space $\left(H^{0}\right)^{\prime}$ which is bigger than $H$. For if we could find $\Lambda \in H$ such that $\gamma \Lambda=\Lambda$ for all $\gamma \in \Gamma$ then the function $b(g)=\langle g \Lambda, \Lambda\rangle$ would be a measurable function of $g$ satisfying $b(\gamma g)=b(g \gamma)=b(g)$, hence by the last result of Mautner [6] $b(g)$ is constant (almost everywhere). It would follow that the given representation of $G$ is the trivial (constant) representation.

If for example $U$ belongs to the principal series, i.e. $U: g \rightarrow U(g, 1 / 2+i t)$ for some real number $t$, then $H$ is naturally isomorphic to $L_{2}(0 \leqq \theta \leqq 1 ; d \theta)$ and $H^{0}$ to the space of all indefinitely differentiable functions $a(\theta)$ on the circumference of the circle with the topology of L. Schwartz. Then $\left(H^{0}\right)^{\prime}$ is the 
space of distributions of $\mathrm{L}$. Schwartz on the circumference of the circle and $\left(H^{0}\right)_{\Gamma}^{\prime}$ consists of those distributions $W$ which satisfy $[d \gamma \theta / d \theta]^{1 / 2+i t} W(\gamma \theta)$ $=W(\theta)$ for all $\gamma \in \Gamma$ and this $t$. A similar interpretation holds for the exceptional series of irreducible unitary representation of $G$ if one replaces $1 / 2+i t$ by $s=$ real $>1 / 2$ and $<1$.

For the discrete series $V_{l}^{+}$the space $\left(H^{0}\right)^{\prime}$ is naturally isomorphic to the space of all power series $\sum_{n \geqq l} b_{n} z^{n}=W(z)$ which satisfy $b_{n}=O\left(n^{p}\right)$ for some $p$. Thus $\left(H^{0}\right)_{\Gamma}^{\prime}$ consists of those $W(z)$ which satisfy also $\left(d \gamma z^{l} / d z\right) W(\gamma z)=W(z)$ i.e. are automorphic forms. Thus in this case $\left(H^{0}\right)^{\prime}$ is naturally isomorphic to a space of automorphic forms of given dimension. For the discrete series $V_{l}^{-}$one has to consider functions $W$ which are regular in $\bar{z}$ instead of $z$. This follows from the explicit description of $V_{l}^{ \pm}$(see Bargmann [1] or $\$ 1$ of Part II).

For the discrete series our Theorem 8.4 has already been obtained by Gelfand and Fomin [1], but not for the other irreducible unitary representations of $G\left({ }^{5}\right)$.

We shall now derive an analogue of the Poisson summation formula. The following argument works for sufficiently differentiable functions, but we shall assume indefinite differentiability for simplicity. Let $f(g) \in D$ $=D(G)$ the space of complex valued indefinitely differentiable functions of compact support on $G$. Then $\sum_{\gamma \in \Gamma} f(g \gamma)$ converges to an indefinitely differentiable function $h(g)$. In fact the mapping

$$
f(g) \rightarrow h(g)=\sum_{\gamma \in \Gamma} f(g \gamma)
$$

is easily seen to be a continuous linear transformation of $D(G)$ into $D(G / \Gamma)$. Let $\psi_{n, j}(g)$ be the complete orthonormal set of eigenfunctions in $L^{2}(G / \Gamma)$ used above. Assume $j$ is such that the corresponding unitary representation $U$ of $G$ is in the principal series $U: g \rightarrow U\left(g, s_{j}\right)$ with $R s_{j}=1 / 2$. Put $H_{m, j}$ $=\int_{G / \Gamma} h(g) \psi_{j, m}(g) d \dot{g}$ where $d \dot{g}$ refers to the invariant measure on $G / \Gamma$ and write again $F_{m n}(s)=\int_{G} f(g) u_{m n}(g, s) d g$. Write $\Lambda_{n, j}=\psi_{n, j}(1)$ and consider the series $\sum_{n} \Lambda_{n, j} \int_{a} f(g) u_{m n}\left(g, s_{j}\right) d g=\sum_{n} \Lambda_{n, j} F_{m n}\left(s_{j}\right)$ for fixed $m$ and $j$. Now $f(g) \in D(G)$ implies by $\S 2$ and 3 of Part II that $F_{m n}(s)$ is for fixed $m$ and $s$ rapidly decreasing in $n$. Now $\left|u_{m n}\left(g, s_{j}\right)\right| \leqq 1$. Hence we may interchange $\sum$ and $\int$ in the last expression. We obtain

$$
\int_{G} f(g) \sum_{n} \Lambda_{n, j} u_{m n}\left(g, s_{j}\right) d g=\sum_{j} \Lambda_{n, j} F_{m n}\left(s_{j}\right) .
$$

But $\sum_{n} \Lambda_{n, j} u_{m n}\left(g, s_{j}\right)=\psi_{j, m}(g)$ by Theorem 8.1 above. Therefore

(5) See also the thesis of F. Bruhat which has appeared in the mean time: Sur les representations induites des groupes de Lie, Paris, Gauthier-Villars, 1956 or Bull. Soc. Math. France vol. 84 (1956) pp. 97-205. 


$$
\begin{aligned}
\int_{G} f(g) \psi_{j, m}(g) d g & =\sum_{n} \Lambda_{n, j} F_{m n}(s) \\
& =\sum_{\gamma \in \Gamma} \int_{Q \gamma^{-1}} f(g) \psi_{j, m}(g) d g=\sum_{\gamma \in \Gamma} \int_{Q} f(g \gamma) \psi_{j, m}(g) d g
\end{aligned}
$$

where $\mathfrak{Q}$ is a fundamental domain for $G / \Gamma$ in $G$. In the last expression we may interchange $\sum_{\gamma}$ with $\int_{\mathfrak{Q}}$ and obtain

$$
\int_{\mathfrak{Q}}\left[\sum_{\gamma} f\left(g \gamma^{h}\right)\right] \psi_{j, m}(g) d g=\int_{\mathfrak{Q}} h(g) \psi_{j, m}(g) d g=\int_{G / \mathbf{\Gamma}} h(g) \psi_{j, m}(g) d \dot{g}=H_{m, j}
$$

This proves

$$
H_{m, j}=\sum_{n} \Lambda_{n, j} F_{m n}\left(s_{j}\right) .
$$

By Theorem 3.1 of Part II we have $F_{m n}\left(s_{j}\right)=O\left(\left[|m|+|n|+1+\left|s_{j}\right|\right]^{-p}\right)$ for every $p$ also $\Lambda_{n, j}=\psi_{j, n}(1)=O\left(\left[n^{2}+\left|s_{j}\left(1-s_{j}\right)\right|\right] r\right)$ for some $r$ by Lemma 8.3 above since $\lambda_{j}=s_{j}\left(1-s_{j}\right)$. Hence

$$
H_{m, j}=O\left(\left[|m|+1+\left|s_{j}\left(1-s_{j}\right)\right|\right]^{-p}\right) \text { for every } p .
$$

Exactly the same arguments and results hold if the $\psi_{j, m}$ span for a given $j$ and varying $m$ a representation space belonging to the discrete or exceptional series except that $F_{m n}(s)$ has to be multiplied by a normalizing function $N_{m n}\left(s_{j}\right)$ which does not change the rapid decrease (see (8.7) above). Now the $\psi_{j, m}$ form a complete orthonormal set in $L^{2}(G / \Gamma)$ hence

$$
h(g)=1 . \text { i.m. } \sum_{j, m} H_{m, j} \Psi_{j, m}(g)
$$

where 1.i.m. refers of course to the $L^{2}(G / \Gamma)$-norm. Now (8.22) and Lemma 8.3 imply that the last series converges uniformly in $g$ for $g$ in any compact subset of $G$. Hence it converges point-wise to $h(g)$. Putting $g=1$ we obtain

$$
\begin{aligned}
\sum_{\gamma \in \Gamma} f(g \gamma) & =h(1)=\sum_{m, j} H_{m, j} \bar{\psi}_{j, m}(1)=\sum_{m, j} H_{m, j} \Lambda_{m, j} \\
& =\sum_{m, n, j} F_{m n}\left(s_{j}\right) N_{m n}\left(s_{j}\right) \bar{\Lambda}_{m, j} \Lambda_{n, j} .
\end{aligned}
$$

This is an analogue of the Poisson summation formula.

9. Modular functions. In $\S 8$ we considered functions on compact homogeneous spaces $G / \Gamma$. In this paragraph we drop the assumption of compactness of $G / \Gamma$, but shall restrict ourselves to the modular subgroup $\Gamma$ of $G$. Let $\tau$ vary over the upper half-plane, i.e. let $\tau$ be any complex number, $\tau=x+i y$ whose imaginary part $y$ is positive. The elements $\gamma$ of $\Gamma$ are given by $2 \times 2$ matrices $\left(M_{i j}\right)$ of determinant one, whose coefficients $M_{i j}$ are rational integers and we put as usual 


$$
\gamma \tau=\frac{M_{11} \tau+M_{12}}{M_{21} \tau+M_{22}}
$$

throughout this paragraph $\Gamma$ denotes the modular group, i.e. the group of modular substitutions $\gamma$ of the form (9.1) of the upper half-plane.

We shall study in this paragraph a certain class of modular functions $h(\gamma \tau)=h(\tau)$ defined below. We shall characterize this class of modular functions. For this purpose we shall use the following well-known Dirichlet series $E(\tau, s)$. We put for any complex number $\tau=x+i y$ with $y>0$ and any complex number $s=\sigma+i t$ with $\sigma>1$

$$
E(\tau, s)=\sum_{M, N ;(M, N) \neq(0,0)} y^{s}|M \tau+N|^{-2 s} .
$$

It is well known that for any fixed $\tau$ with $y>0$ this function of $s$ has an analytic continuation into the whole $s$-plane which is regular in $s$ everywhere except at $s=1$ where it has a pole of order 1 and satisfies the functional equation

$$
\pi^{-s} \Gamma(s) E(\tau, s)=\pi^{s-1} \Gamma(1-s) E(\tau, 1-s) .
$$

We shall need the following properties of $E(\tau, s)$.

LEMMA 9.1.

$$
E(\tau, s)=y^{s} \zeta(2 s)+y^{1-s} \pi^{1 / 2} \frac{\Gamma(s-1 / 2)}{\Gamma(s)} \zeta(2 s-1)+R(\tau, s)
$$

where

$$
R(\tau, s)=y^{1 / 2} \frac{2 \pi^{s}}{(s)} \sum_{n \neq 0} b_{n}(s)|n|^{s-1 / 2} K_{z-1 / 2}(2 \pi|n| y) e^{2 \pi i n x}
$$

The function $R(\tau, s)$ is for each fixed $\tau$ in the upper half plane an entire function of $s$. Here $b_{n}(s)=\sum_{m \mid n} e^{2 \pi i n / m}|m|^{1-2 s}, K_{s}(z)$ is the modified Bessel function of the third kind, defined for example by

(9.4) $K_{s-1 / 2}(z)=\pi^{1 / 2}\left(\frac{z}{2}\right)^{s-1 / 2} \Gamma(s)^{-1} \int_{1}^{\infty} e^{-z t}\left(t^{2}-1\right)^{s-1} d t$ for $R s>0, R z>0$ and $\zeta(s)$ is the Riemann zeta function.

This lemma is well known; its proof can be found for instance in Maass [1].

Lemma 9.2. If $\zeta(s)$ denotes the Riemann zeta function, then for $\sigma \geqq 1 / 2$, $|s-1|>c$

$$
\zeta(s)=O(|s|)
$$




$$
\frac{1}{\zeta(1+i t)}=O\left(\log ^{7} t\right)
$$

Also $\zeta(s)=O\left(|t|^{3 / 2+\delta}\right)$ for any $\sigma \geqq-\delta$, as $|t| \rightarrow \infty$.

For the proof of these well known facts see for instance Titchmarsh [3, p. 29 (2.12.2), p. $44(3.6 .5)$ and p. 81 (5.1.1)] respectively.

Lemma 9.3. $\left|K_{\lambda+i \mu}(y)\right| \leqq C_{c, \lambda} y^{-c} e^{-\pi|\mu| / 2}\left(1+|\mu|^{c+\lambda}\right)$ for all $y>0$, all real numbers $\lambda$ and $\mu$ and all $c>1+|\lambda|$; here $C_{c, \lambda}$ is a constant depending only on $c$ and $\lambda$.

Proof. We have the well known formula $K_{v}(z)=\int_{0}^{\infty} e^{-z \cosh x} \cosh (v x) d x$ for $R z>0$. For $v=i t$ we get

$$
\begin{aligned}
K_{i t}(z) & =\int_{0}^{\infty} e^{-z / 2}\left(e^{x}+e^{-x}\right) \frac{e^{i t x}+e^{-i t x}}{2} d x \\
& =\frac{1}{2} \int_{-\infty}^{\infty} e^{-z / 2}\left(e^{x}+e^{-x}\right) e^{i t x} d x .
\end{aligned}
$$

Now it is well known that

$$
\int_{0}^{\infty} K_{v}(x) x^{s-1} d x=2^{s-2} \Gamma\left(\frac{s}{2}-\frac{v}{2}\right) \Gamma\left(\frac{s}{2}+\frac{v}{2}\right) \text { for } R s>|R v| .
$$

Hence

$$
K_{v}(x)=\frac{1}{2 \pi i} \int_{c-i \infty}^{c+i \infty} 2^{s-2} \Gamma\left(\frac{s}{2}-\frac{v}{2}\right) \Gamma\left(\frac{s}{2}+\frac{v}{2}\right) x^{-s} d s
$$

where $s=c+i y$ and $v=\lambda+i \mu$, hence

$$
\begin{aligned}
\left|K_{v}(x)\right| \leqq & \frac{1}{2 \pi} \int_{c-i \infty}^{c+i \infty} 2^{c-2}\left|\Gamma\left(\frac{s}{2}-\frac{v}{2}\right) \Gamma\left(\frac{s}{2}+\frac{v}{2}\right)\right| x^{-c}|\cdot| d s \mid \\
= & \text { const. } x^{-c} \int_{-\infty}^{\infty}\left|\Gamma\left(\frac{c-\lambda+i(y-\mu)}{2}\right) \Gamma\left(\frac{c+\lambda+i(y+\mu)}{2}\right)\right| d y \\
& \cdot \int_{-\infty}^{\infty}\left[1+\left|\frac{\eta^{2}-\mu^{2}}{4}\right|^{(c+\lambda-1) / 2}\right] e^{-\pi / 4(|\eta+\mu|+|\eta-\mu|)} d \eta .
\end{aligned}
$$

Assume first that $\mu \geqq 0$. Then

$$
\int_{-\infty}^{\infty}=\int_{-\mu}^{-\mu}+\int_{\mu}^{\infty}+\int_{-\mu}^{\mu}
$$

Now, 


$$
\begin{aligned}
\int_{-\infty}^{-\mu} & =\int_{-\infty}^{\mu}\left[1+\left|\frac{\eta^{2}-\mu^{2}}{4}\right|^{(c+\lambda-1) / 2}\right] e^{-\pi / 4(-\eta-\mu+\mu-\eta)} d \eta \\
& =\int_{-\infty}^{-\mu}\left[1+\left|\frac{\eta^{2}-\mu^{2}}{4}\right|^{(c+\lambda-1) / 2}\right] e^{+\pi \eta / 2} d \eta \\
& \leqq \text { const. }{ }_{c+\lambda} e^{-\pi \mu / 2}\left\{\mu^{c+\lambda}+1\right\} .
\end{aligned}
$$

Also

$$
\begin{aligned}
\int_{\mu}^{\infty} & =\int_{\mu}^{\infty}\left[1+\left|\frac{\eta^{2}-\mu^{2}}{4}\right|^{(c+\lambda-1) / 2}\right] e^{-\pi / 4(\eta+\mu+\eta-\mu)} d \eta \\
& =\int_{\mu}^{\infty}\left[1+\left|\frac{\eta^{2}-\mu^{2}}{4}\right|^{(c+\lambda-1) / 2}\right] e^{-\pi \eta / 2} d \eta \\
& \leqq \text { const. } . c+\lambda e^{-\pi \mu / 2}\left\{\mu^{c+\lambda}+1\right\} .
\end{aligned}
$$

Finally

$$
\begin{aligned}
\int_{-\mu}^{\mu} & =\int_{-\mu}^{\mu}\left[1+\left|\frac{\eta^{2}-\mu^{2}}{4}\right|^{(c+\lambda-1) / 2}\right] e^{-\pi / 4(\eta+\mu+\mu-\eta)} d \eta \\
& =e^{-\pi \mu / 2} \int_{-\mu}^{\mu}\left[1+\left(\frac{\eta^{2}-\mu^{2}}{4}\right)^{(c+\lambda-1) / 2}\right] d \eta \\
& \leqq \text { const. }{ }_{c+\lambda} e^{-\pi \mu / 2}\left\{\mu^{c+\lambda}+1\right\} .
\end{aligned}
$$

Now $|\Gamma(x+i y)| \leqq$ const. $x|y+1|^{x-1 / 2} e^{\pi|y| / 2}$ for $x>\epsilon$, hence

$$
\left|\Gamma\left(\frac{c \pm \lambda}{2}+i \frac{y \pm \mu}{2}\right)\right| \leqq \text { const. } c, \lambda\left|\frac{y \pm \mu}{2}+1\right|^{(c+\lambda) / 2-1 / 2} e^{-(x / 2)(\mid y \pm \mu / 2) \mid}
$$

$$
\text { for } c \pm \lambda>0 \text {. }
$$

Hence

$$
\begin{aligned}
& \left|K_{\lambda+i \mu}(x)\right| \\
& \quad \leqq \text { const. }, \lambda x^{-c} \int_{-\infty}^{\infty}\left|\frac{y^{2}-\mu^{2}}{4}+1\right|^{(c+\lambda-1) / 2} e^{-x / 4}(|y+\mu|+|y-\mu|) d y
\end{aligned}
$$

where the const. $c, \lambda$ depends on $c$ and $\lambda$ and this inequality holds for all $x>0$, all $\lambda, \mu$, and $c$ provided $c>1-\lambda$ and $c>|\lambda|$, hence certainly for $c>1+|\lambda|$.

Hence,

$$
\left|K_{\lambda+i \mu}(y)\right| \leqq \text { const. } c, \lambda^{-c} y^{-\pi|\mu| / 2}\left(1+|\mu|^{c+\lambda}\right)
$$

for all $y>0$, all $\lambda$ and $\mu$, all $c>1+|\lambda|$, as required.

We shall also need an estimate of the function $R(\tau, s)$ defined in Lemma 9.1. We have 
LemмA 9.4. $|R(\tau, s)| \leqq c_{p, \sigma} y^{1 / 2-p}\left(1+|t|^{p+|2 \sigma|}\right)$ for all $\tau=x+i y, y \geqq \epsilon>0$, for all $s=\sigma+i$ and all sufficiently large $p$. Here $c_{p \sigma}$ is a constant depending only on $p$ and $\sigma$ (and $\epsilon$ ) which can be taken to be independent of $p$ and $\sigma$, provided $p$ and $\sigma$ are in finite intervals.

Proof. By Lemma 9.1

$$
R(\tau, s)=\frac{2 \pi^{s} y^{1 / 2}}{\Gamma(s)} \sum_{n \neq 0} b_{n}(s)|n|^{s-1 / 2} K_{s-1 / 2}(2 \pi|n| y) e^{2 \pi i n x},
$$

hence

$$
\begin{aligned}
|R(\tau, s)| \leqq & \frac{2 \pi}{|\Gamma(s)|} y^{1 / 2} \sum_{n \neq 0}\left|b_{n}(s)\right||n|^{\sigma-1 / 2} \text { const.c,o }(2 \pi|n| y)^{-p} \\
& \cdot e^{-\pi|t| / 2}\left(1+|t|^{p+\sigma-1 / 2}\right) \\
= & \text { const.p, } \sigma \frac{y^{1 / 2-p}}{|\Gamma(s)|} e^{-\pi|t| / 2}\left(1+|t|^{p+\sigma-1 / 2}\right) \sum_{n \neq 0}\left|b_{n}(s)\right||n|^{\sigma-1 / 2-p} .
\end{aligned}
$$

Now $\sum_{n \neq 0}\left|b_{n}(s)\right||n|^{\sigma-1 / 2-p}$ is convergent for $p$ large enough and thus is dominated by a constant (depending on $p$ and $\sigma$ ). Hence for all $\tau$ and $s$

$$
\begin{aligned}
& |R(\tau, s)| \leqq \text { const. } p, \sigma \frac{y^{1 / 2-c}}{|\Gamma(s)|} e^{-\pi|t| / 2}\left(1+|t|^{p+\sigma-1 / 2}\right) \\
& \leqq \text { const.p, } y^{1 / 2-p}\left(|t|^{-\sigma+1 / 2}+1\right)\left(1+|t|^{p+\sigma-1 / 2}\right)
\end{aligned}
$$

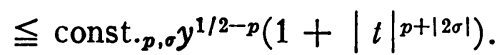

LEMma 9.5. $|(s-1) E(\tau, s)| \leqq$ const. $\left(1+y^{r}\right)\left(1+|t|^{r}\right)$ for some $r$, but all $\tau$ and $s$. The constant depends only on $\sigma$ and can be taken to be independent of $\sigma$ if $\sigma$ stays in a finite interval.

Proof. $\left|(s-1 / 2) y^{s} \zeta(2 s)\right| \leqq$ const. $\left(1+|t|^{\sigma / 2+\delta}\right) y^{\sigma}$ for $\sigma \geqq-\delta$ by Lemma 9.2.

$$
\begin{array}{r}
\left|(s-1)(s-1 / 2) y^{1-s} \frac{\Gamma(s-1 / 2)}{\Gamma(s)} \zeta(2 s-1)\right| \leqq \text { const. }\left(1+|t|^{8+\delta}\right) y^{1-\sigma} \\
\quad \text { for } \sigma \geqq \frac{1}{2}-\frac{\delta}{2}
\end{array}
$$

by Lemma 9.2 and Sterling's formula. Combining these inequalities with Lemmas 9.1 and 9.4 proves Lemma 9.5 .

Let $\mathfrak{Q}$ denote a fundamental domain for the modular group in the upper half plane. For the sake of definiteness let $\mathfrak{Q}$ be defined by

$$
-\frac{1}{2}<x \leqq \frac{1}{2}, \quad|\tau|=|x+i y| \geqq 1 .
$$


Definition 9.1. Let $A$ denote the class of all complex valued functions $h(\tau)$ defined in the upper half plane of complex numbers $\tau=x+i y, y>0$ which are

(i) indefinitely differentiable in $x$ and $y$,

(ii) $\left(\Delta^{i} h\right)(\tau)=O\left(y^{-p}\right)$ for all non-negative integers $j$ and $p$ as $y \rightarrow+\infty$ uniformly in $x$ where

$$
\Delta=-y^{2}\left(\frac{\partial^{2}}{\partial x^{2}}+\frac{\partial^{2}}{\partial y^{2}}\right) .
$$

(iii) $h(\tau)$ is invariant under the modular group, i.e.,

$$
h\left(\left(M_{11} \tau+M_{12}\right) /\left(M_{21} \tau+M_{22}\right)\right)=h(\tau)
$$

for any integers $M_{i j}$ with $M_{11} M_{22}-M_{12} M_{21}=1$.

(iv) $\iint_{\mathscr{Q}} h(\tau) \phi_{n}(\tau) d x d y / y^{2}=0$ for all $n=0,1,2, \ldots$

where $\phi_{0}=$ constant, $\phi_{1}, \phi_{2}, \cdots$ are the eigenfunctions in the point spectrum of $\Delta$ acting on $L_{2}\left(\mathfrak{Q} ; y^{-2} d x d y\right): \Delta \phi_{n}=\lambda_{n} \phi_{n}, \quad \lambda_{0}=0<\lambda_{1} \leqq \lambda_{2} \leqq \cdots, \phi_{n}$ $\in L_{2}\left(\mathfrak{Q} ; y^{-2} d x d y\right)$.

Let $B$ denote the class of all complex valued functions $H(s)$ of a complex variable $s=\sigma+i$ such that

(I) $(s-1) H(s)$ is an entire function of $s$,

(II) $\pi^{-8} \Gamma(s) H(s)=\pi^{s-1} \Gamma(1-s) H(1-s)$,

(III) $(s-1) H(s)=0\left(|t|^{-p}\right)$ for all $p$ as $|t| \rightarrow \infty$, uniformly for $\sigma$ in any finite interval.

Lemma 9.6. If $h(\tau) \in A$, then $H(s)=\iint \propto h(\tau) E(\tau, s) d x d y / y^{2} \in B$.

Proof. By the definition of $\mathrm{A}$ and Lemma 9.5 we have

$$
|h(\tau)(s-1) E(\tau, s)| \leqq \text { const. } y^{-p} y^{r}(1+|t| r)
$$

for all $p$ and some $r$, if $\tau \in \mathfrak{Q}$. Thus

$$
|h(\tau)(s-1) E(\tau, s)| \leqq \text { const. } \frac{1+|t| r}{|y|^{p-r}} .
$$

Hence $\iint_{\mathscr{O}} h(\tau)(s-1) E(\tau, s) d x d y / y^{2}$ converges uniformly in $s$ if $s$ varies over a compact set. Therefore $(s-1) H(s)$ is entire in $s$. Moreover $H(s)$ clearly satisfies the same functional equation as $E(\tau, s)$. Thus $H(s)$ satisfies (I) and (II) in the definition of $B$.

To verify (III) note that (9.7) implies

$$
|(s-1) H(s)| \leqq \text { const. } \sigma(1+|t| r) \text {. }
$$

Now $\left(\Delta^{i} h\right)(\tau)=h_{j}(\tau) \in A$ whenever $h(\tau) \in A$ where $\Delta$ is defined by (9.6). Moreover $\left(\Delta^{i} E\right)(\tau, s)=s^{i}(1-s)^{i} E:(\tau, s)$ hence

$$
\iint_{Q} h_{j}(\tau) E(\tau, s) \frac{d x d y}{y^{2}}=s^{j}(1-s)^{i} H(s) .
$$


Now apply (9.8) to $h_{j}(\tau)$ instead of $h(\tau)$. We have

$$
\left|s^{i}(1-s)^{j+1} H(s)\right| \leqq \text { const. } \sigma, j(1+|t| r)
$$

for one suitable $r$, and all positive integers $j$. Therefore $H(s)$ satisfies also (III). This proves Lemma 9.6.

Lemma 9.7. $\bar{E}(\tau, s)=E(\tau, \bar{s})$ for all $s(\neq 1)$. Hence

$$
\bar{E}(\tau, 1 / 2+i t)=E(\tau, 1 / 2-i t) .
$$

Proof. For $R s>1$ the equation $\bar{E}(\tau, s)=E(\tau, \bar{s})$ follows at once from the convergence of the Dirichlet series $\sum y^{s}|M \tau+N|^{-2}$, for all other $s(\neq 1)$ by analytic continuation.

Now put, for $H(s) \in B$

$$
\begin{aligned}
& H_{1}(y, s)=\frac{H(s) y^{1-\sigma}}{\zeta(2 s)} \\
& H_{2}(y, s)=\pi^{1 / 2} \frac{\Gamma(1 / 2-s)}{\Gamma(1-s)} H(s) \frac{\zeta(1-2 s)}{\zeta(2 s) \zeta(2-2 s)} y^{8} .
\end{aligned}
$$

Now we use Riemann's functional equation for $\zeta(2 s)$, i.e.,

$$
\frac{\zeta(1-2 s)}{\zeta(2 s)}=\frac{2^{-2 s} \pi^{1-2 s}}{\sin \pi s \Gamma(1-2 s)}
$$

and get

$$
\begin{aligned}
H_{2}(y, s) & =\frac{\Gamma(1 / 2-s)}{\Gamma(1-s) \Gamma(1-2 s)} \frac{2^{-2 s} \pi^{3 / 2-2 s}}{\sin \pi s} \frac{H(s) y^{s}}{\zeta(2-2 s)} \\
& =\frac{\Gamma(s) \Gamma(1 / 2-s)}{\Gamma(1-2 s)} 2^{-2 s} \pi^{1 / 2-2 s} \frac{H(s) y^{s}}{\zeta(2-2 s)} .
\end{aligned}
$$

Now we use

$$
\Gamma(1-2 s)=-2 s \Gamma(-2 s)=-2 s 2^{-2 s-1} \pi^{-1 / 2} \Gamma(-s) \Gamma(1 / 2-s)
$$

and get

$$
H_{2}(y, s)=-\frac{\pi^{1-2 s} \Gamma(s)}{s \Gamma(-s)} \frac{H(s) y^{s}}{\zeta(2-2 s)}=\frac{\pi^{1-2 s} \Gamma(s) H(s) y^{s}}{\Gamma(1-s) \zeta(2-2 s)} .
$$

On the other hand

$$
H_{1}(y, 1-s)=\frac{H(1-s) y^{s}}{\zeta(2-2 s)}=\pi^{1-2 s} \frac{\Gamma(s)}{\Gamma(1-s)} \frac{H(s) y^{s}}{\zeta(2-2 s)}
$$

using the functional equation for $H(s)$. This proves 
Lemma 9.8. If $H(s)$ satisfies the functional equation (II), then $H_{1}(y, 1-s)$ $=H_{2}(y, s)$.

Lemma 9.9. If $H(s) \in B$, then for any real number $r>0, \int_{R_{s=1 / 2}} H_{1}(y, s) d s$ $=\int_{R_{s=1 / 2+r}} H_{1}(y, s) d s$ and $\int_{R_{s=1 / 2}} H_{2}(y, s) d s=\int_{R s-1 / 2-r} H_{2}(y, s) d s$. All integrals exist absolutely.

Proof. By hypothesis $H(s)$ is rapidly decreasing along any vertical line. Also by Lemma $9.2(1 / \zeta)(1+i t)=O(t)$, hence $\int_{R s=1 / 2} H_{1}(y, s) d s$ converges absolutely. Since $H_{2}(y, 1 / 2-i t)=H_{1}(y, 1 / 2+i t)$, the same is true for $H_{2}(y, s)$. Moreover for fixed $\sigma>1 / 2$ the function $(1 / \zeta)(2+2 i t)$ is bounded in $t$, so that $\int_{R s=1 / 2+r} H_{1}(y, s) d s$ converges absolutely for any $r \geqq 0$. Hence using again $H_{2}(y, 1-s)=H_{1}(y, s)$ (Lemma 9.7 above) we see that $\int_{R s=1 / 2-r} H_{2}(y, s) d s$ converges absolutely for any $r \geqq 0$.

The equality of the integrals containing $H_{1}(y, s)$ follows from the fact that in the strip $1 / 2 \leqq R s \leqq 1 / 2+r$ the function $H_{1}(y, s)$ is for each fixed $y>0$ regular analytic in $s$ and rapidly decreasing in $t$ uniformly in $\sigma$. Similarly for $H_{2}(y, s)$.

Now put

$$
h_{j}(y)=\int_{R_{s=1 / 2}} H_{j}(y, s) d s \quad \text { for } j=1,2 .
$$

Lemma 9.10. If $H(s) \in B$ then $h_{j}(y)=O\left(y^{-p}\right)$ as $y \rightarrow+\infty$ for all $p>0$. The above integral converges absolutely and uniformly in $y$ for $0<\epsilon \leqq y \leqq 1 / \epsilon$.

This is an immediate consequence of Lemma 9.9 and the above properties of $H_{j}(y, s)$.

Now put

$$
h_{3}(\tau)=\int_{R s=1 / 2} H(s) R(\tau, 1-s) \frac{d s}{|\zeta(2 s)|^{2}}
$$

Then we have

Lemma 9.11. If $H(s) \in B$ then $h_{3}(\tau)=O\left(y^{-p}\right)$ as $y \rightarrow+\infty$ for any $p>0$. The integral converges again absolutely and uniformly in $\tau$ for $0<\epsilon \leqq y \leqq 1 / \epsilon$.

This follows from Lemmas 9.2 and 9.4 and condition (III) in the definition of the class $\mathrm{B}$.

Finally we have

Lemma 9.12. If $H(s) \in B$ then $\int_{R_{s=1 / 2}} H(s) E(\tau, 1-s)|\zeta(2 s)|^{-2} d s$ satisfies conditions (i), (ii) and (iii) of Definition 9.1 above.

Proof. Put $h(\tau)=h_{1}(\tau)+h_{2}(\tau)+h_{3}(\tau)$. Then by the definition of $h_{j}(\tau)$ and Lemma 9.1 we have 


$$
h(\tau)=\int_{R s=1 / 2} H(s) E(\tau, 1-s) \frac{d s}{|\zeta(2 s)|^{2}} .
$$

Since $E(\tau, s)$ is for each fixed $s \neq 1$ an invariant function of $\tau$ under the modular group, this shows that $h(\tau)$ satisfies condition (iii) in the Definition 9.1 of the class $\mathrm{A}$.

By Lemmas 9.10 and 9.11, the integral (9.14) converges uniformly in $\tau$ for $\tau$ in any compact subset of the upper half plane. Now $\Delta E(\tau, s)=s(1-s)$ $\cdot E(\tau, s)$, hence

$$
\begin{aligned}
\int_{R_{s=1 / 2}} H(s) \Delta^{l} E(\tau, 1-s) \frac{d s}{|\zeta(2 s)|^{2}} & \\
& =\int_{R_{s=1 / 2}} s^{l}(1-s)^{l} H(s) E(\tau, 1-s) \frac{d s}{|\zeta(2 s)|^{2}}
\end{aligned}
$$

converges uniformly in $\tau$ for $\tau$ in compact sets, because $s^{l}(1-s)^{l} H(s) \in B$ for any integer $l \geqq 0$ whenever $H(s) \in B$. This proves that $h(\tau)$ is indefinitely differentiable ( $\Delta$ being elliptic) and $\left(\Delta^{l} h\right)(\tau)$ is equal to the last integral. This proves that $h(\tau)$ satisfies condition (i) of the definition of the class A. Condition (ii) is satisfied because on applying Lemmas 9.10 and 9.11 with $s^{l}(1-s)^{l} H(s)$ instead of $H(s)$ we see that $\left(\Delta^{l} h\right)(\tau)=O\left(y^{-p}\right)$ for all $p$ and $l>0$ as $y \rightarrow+\infty$.

Let $\phi_{0}, \phi_{1}, \cdots$ be again the eigenfunctions in the point spectrum of $\Delta$ acting on $L_{2}\left(\mathfrak{Q} ; y^{-2} d x d y\right)$ introduced above. We normalize them so that

$$
\int_{\mathfrak{Q}} \phi_{j}(\tau) \bar{\phi}_{l}(\tau) \frac{d x d y}{y^{2}}=\delta_{j l}
$$

and $\phi_{0}(\tau)$ constant. A result of Roelcke [1] states that

$$
\phi_{j}(x+i y)=O\left(y^{c}\right) \text { as } y \rightarrow+\infty \text { for some constant } c .
$$

We shall prove

Lemma 9.13. Given any real number $p>0$, then there exists a constant $c_{p}$ independent of $x$ such that

$$
\left|\phi_{j}(\tau)\right| \leqq c_{p}\left(1+\left|\lambda_{j}\right|^{(p+1) / 2}\right) y^{-p} \text { for } y \geqq 1 .
$$

Proof. Using the above result of Roelcke, we see that our $\phi_{j}$ satisfy the assumptions which Maas [1] makes about his "automorphic wave-functions." Hence we may use formula (61) of Maas [1] which states that for $n \neq 0$ and $j \neq 0$

$$
\int_{-1 / 2}^{1 / 2} \phi_{j}(x+i y) e^{-2 \pi i u x} d x=C_{n, j} y^{1 / 2} K_{i r_{j}}(2 \pi|n| y)
$$

where the $C_{n, j}$ are constants and $1 / 4+r_{j}^{2}=\lambda_{j}$. By Schwarz's inequality 


$$
\left|C_{n, j} y^{1 / 2} K_{i r_{j}}(2 \pi|n| y)\right|^{2} \leqq \int_{-1 / 2}^{1 / 2}\left|\phi_{j}(x+i y)\right|^{2} d x
$$

hence

$$
\left|C_{n, j}\right|^{2} \int_{1 /|n|}^{\infty}\left|K_{i r_{j}}(2 \pi|n| y)\right|^{2} \frac{d y}{y} \leqq \int_{1 /|n|}^{\infty} \int_{-1 / 2}^{1 / 2}\left|\phi_{j}(x+i y)\right|^{2} \frac{d x d y}{y^{2}} .
$$

Now the number of fundamental domains of the modular group which intersect the rectangle $-1 / 2 \leqq x \leqq 1 / 2,1 /|n| \leqq y$ is $O(n)$. Hence

$$
\int_{1 /|n|}^{\infty} \int_{-1 / 2}^{1 / 2}\left|\phi_{j}(x+i y)\right|^{2} \frac{d x d y}{y^{2}}=O(n)
$$

uniformly in $j \geqq 1$.

On the other hand, it is known that

$$
\int_{1 /|n|}^{\infty}\left|K_{i r_{j}}(2 \pi|n| y)\right|^{2} \frac{d y}{y}=\int_{1}^{\infty}\left|K_{i r_{j}}(2 \pi y)\right|^{2} \frac{d y}{y} \geqq c \frac{e^{-\pi\left|r_{j}\right|}}{\left(1+\frac{\pi}{2}\left|r_{j}\right|\right)^{2}}
$$

where $c$ is a constant. Hence

$$
\left|C_{n, j}\right| \leqq c^{\prime}|n|\left(1+\frac{\pi}{2}\left|r_{j}\right|\right) e^{\pi\left|r_{j}\right| / 2} \text { for }|n|>0 \text { and } j>0 .
$$

Now according to Maas [1] one has for $j \neq 0$,

$$
\phi_{j}(\tau)=\sum_{n \neq 0} C_{n, j} K_{i r_{j}}(2 \pi|n| y) e^{2 \pi i n x} .
$$

Let us use Lemma 9.3, and we see that

$$
\begin{aligned}
\left|\phi_{j}(\tau)\right| & \leqq \sum_{n \neq 0}|n|\left(1+\frac{\pi}{2}\left|r_{j}\right|\right) e^{\pi\left|r_{j}\right| / 2} c_{p}(|n| y)^{-p} e^{-\pi\left|r_{j}\right| / 2}\left(1+\left|r_{j}\right|^{p}\right) \\
& =c_{p}^{\prime} y^{-p}\left(1+\left|r_{j}\right|^{p+1}\right) \sum_{n \neq 0}|n|^{1-p} .
\end{aligned}
$$

This completes the proof of Lemma 9.13.

Combining the above with Satz 17 of Roelcke [1] (which states that $\sum_{j}\left|\lambda_{j}\right|^{-2}$ is convergent) we obtain

COROLlaRy. If $a_{j}$ is any rapidly decreasing sequence of complex numbers, i.e., $a_{j}=O\left(j^{-p}\right)$ for any real $p$, then

$$
\sum_{j=1} a_{j} \phi_{j}(\tau)
$$

satisfies conditions (i), (ii) and (iii) in the definition of the class A.

Conversely, if $h(\tau)$ satisfies (i), (ii) and (iii), in the definition of the class $A$, then 


$$
\iint_{Q} h(\tau) \bar{\phi}_{j}(\tau) \frac{d x d y}{y^{2}}=O\left(j^{-p}\right)
$$

as $j \rightarrow+\infty$.

Let us now drop the assumption (iv) in the definition of the class A.

Definition 9.2. Let $\mathfrak{A}$ be the direct sum of the constants with the space $\mathfrak{Y}$ of all those functions $h(\tau)$ defined in the upper half plane $\tau=x+i y, y>0$ which satisfy conditions (i), (ii) and (iii) in the Definition 9.1 of the class A, and

$$
\iint_{\mathbb{Q}} h(\tau) y^{-2} d x d y=0
$$

We topologize $\mathfrak{A}^{\prime}$ by means of the semi-norms

$$
\sup _{\tau \in \mathbb{Q}}\left|y^{p}\left(\Delta^{i} h\right)(\tau)\right|, \quad j, p=0,1,2, \cdots
$$

It is readily verified that $\mathfrak{A}$ is a metrizable complete locally convex topological vector space, i.e., $\mathfrak{A}$ is a Frechet space.

LEMmA 9.14. Let $h(\tau) \in \mathfrak{A}$ and put $H(s)=\iint \mathfrak{Q} h(\tau) E(\tau, s) y^{-2} d x d y$. Then

$$
\lim _{s \rightarrow 1}(s-1) H(s)=\frac{\pi}{2} \iint_{Q} h(\tau) y^{-2} d x d y .
$$

Proof. By Lemma 9.1 we have

$$
\begin{aligned}
\lim _{s \rightarrow 1}(s-1) E(\tau, s) & =\lim _{s \rightarrow 1}\left[y^{s} \zeta(2 s)+y^{1-s} \pi^{1 / 2} \frac{\Gamma(s-1 / 2)}{\Gamma(s)} \zeta(2 s-1)+R(\tau, s)\right] \\
& =\pi^{1 / 2} \frac{\Gamma(1 / 2)}{\Gamma(1)} \lim _{s \rightarrow 1}(s-1) \zeta(2 s-1)=\frac{\pi}{2} .
\end{aligned}
$$

Now if $h(\tau)$ is orthogonal to the constants then $h(\tau)=O\left(y^{-p}\right)$ for every $p$ by definition of $\mathfrak{A}$ and $(s-1) E(\tau, s)=O\left(y^{r}\right)$ for some $r$ if $s$ is in a compact set by Lemma 9.5, so that $(s-1) h(\tau) E(\tau, s)$ is bounded if $s$ is in a compact set. Moreover $\iint_{0 y^{-2}} d x d y<\infty$ as is well known and readily verified. Hence we may apply the Lebesgue convergence theorem and obtain the assertion of Lemma 9.14, if $h(\tau)$ is orthogonal to the constants. If $h(\tau)$ is constant, Lemma 9.4 is readily verified.

We now introduce the space $\mathfrak{B}$ :

Definition 9.3. Let $\mathfrak{A}$ be the set of all pairs $\left\{H(s), a_{j}\right\}$ where $H(s)$ is in the class $B$ of Definition 9.1 and $a_{j}(j=0,1,2, \cdots)$ an arbitrary rapidly decreasing sequence, satisfying

$$
\lim _{s \rightarrow 1}(s-1) H(s)=\frac{\left(\pi^{3}\right)^{1 / 2}}{2 \cdot 3^{1 / 2}} a_{0} .
$$


We topologize $\mathfrak{B}$ by means of the semi-norms

$$
\text { (9.17) } \max _{|\sigma-1 / 2| \leqq q}\left\{\left(1+|t|^{p}\right)|(1-s) H(s)|\right\}, \quad \max _{j}\left\{(1+j)^{r}\left|a_{j}\right|\right\}
$$

for any positive $p, q, r$. Again $\mathfrak{B}$ is a Frechet space.

Let us now consider the mapping

$$
h(\tau) \rightarrow\left\{H(s), a_{j}\right\}
$$

where

$$
H(s)=\iint_{Q} h(\tau) E(\tau, s) y^{-2} d x d y
$$

and

$$
a_{j}=\iint_{\mathfrak{Q}} h(\tau) \Phi_{j}(\tau) y^{-2} d x d y
$$

Theorem 9.1. The mapping (9.18) is a topological isomorphism of the topological vector space $\mathfrak{A}$ (of Definition 9.2 ) onto the topological vector space $\mathfrak{B}$ (of Definition 9.3).

Proof. The mapping (9.18) is clearly linear. By Lemmas 9.6 and 9.14, and the corollary after Lemma $9.13,(9.18)$ maps $\mathfrak{A}$ into $\mathfrak{B}$. Now we use the result A. Selberg (Lectures, Institute for Advanced Study, 1954-1955) and W. Roelcke $[1,(140)]$ that $(9.18)$ is a unitary mapping of $L^{2}\left(\mathfrak{Q}: y^{-2} d x d y\right)$ onto the direct sum of $L^{2}\left(R s=1 / 2 ; d s /|\zeta(2 s)|^{2}\right)$ with Hilbert space $l^{2}$ of all square summable sequences $a_{j}$. Thus if $\left\{H(s), a_{j}\right\} \in \mathfrak{B}$ then its inverse image under (9.18) is given by

$$
h(\tau)=\int_{R_{s=1 / 2}} H(s) E(\tau, 1-s) \frac{d s}{|\zeta(2 s)|^{2}}+\sum_{j=0} a_{j} \phi_{j}(\tau) .
$$

Now we apply Lemmas 9.12, 9.14 and the corollary to Lemma 9.13 and conclude that (9.18) maps $\mathfrak{A}$ one-one onto $\mathfrak{B}$ as follows: suppose $H(s) \in \mathfrak{B}$ and put $h^{1}(\tau)=\int_{R_{s=1 / 2}} H(s) E(\tau, 1-s)|\zeta(2 s)|^{-2} d s$, repeat the argument used in the proof of Lemma 9.12. One finds that $h^{1}(\tau)+a_{0} \in \mathfrak{A}$ (where the additional term $a_{0}$ comes from the residues at the poles $s=0$ or 1 obtained by shifting the line of integration as in the proof of Lemma 9.9). It follows that $h(\tau)$ as defined by (9.19) is contained in $\mathfrak{A}$.

Since the topology of $\mathfrak{A}$ is stronger than that of $L^{2}\left(\mathfrak{Q}: y^{-2} d x d y\right)$ we con. clude that (9.18) is a closed mapping of $\mathfrak{A}$ onto $\mathfrak{B}$. Since $\mathfrak{A}$ and $\mathfrak{B}$ are Frechet spaces, the closed graph theorem applies (see Bourbaki [1]) so that (9.18) is continuous in the topologies of $\mathfrak{A}$ and $\mathfrak{B}$. The continuity of the inverse of (9.18) follows in the same way. This proves Theorem 9.1. 
Corollary. Let $s$ be any fixed complex number $\neq 1,0,-1,-2, \cdots$. Then $E(\tau, s)$ is not identically zero in $\tau$.

\section{BIBLIOGRAPHY}

\section{BARGMANN}

1. Unitary representations of the Lorentz group, Ann. of Math. vol. 48 (1947) pp. 568-640. A. Beurling

1. On two problems concerning linear transformations in Hilbert space, Acta Math. vol. 81

N. BOURBAKI (1948) pp. 239-255.

1. XV, Livre V. Espaces vectoriels topologiques, Paris, Hermann, 1953.

2. XIII, Livre 6. Integration, Chapter III, Paris, Hermann, 1953.

J. Dieudonnt and L. Schwartz

1. La dualite dans les Espaces $(F)$ et $(L F)$, Ann. Institut Fourier, Grenoble vol. 1 (1950) pp. 61-101.

L. EHRENPREIS

1. Theory of distributions for locally compact spaces, Part I, Columbia University Thesis, 1953; Memoirs of the Amer. Math. Soc., no. 21, 1956, 80 pp.

2. Some properties of distributions on Lie groups, Pacific J. Math. vol. 6 (1956) pp. 591-606.

3. Solutions of some problems of division I, Amer. J. Math. vol. 76 (1954) pp. 883-903.

4. Analytic functions and the Fourier transform of distributions I, Ann. of Math. vol. 63 (1956) pp. 129-159.

5. Mean periodic functions I, Amer. J. Math. vol. 78 (1955) pp. 292-328.

L. EhrenPreis and F. I. Mautner

1. Some properties of the Fourier transform on semi-simple Lie groups, I, Ann. of Math. vol. 61 (1955) pp. 406-439.

2. Some properties of the Fourier transform on semi-simple Lie groups, II, Trans. Amer. Math. Soc. vol. 84 (1957) pp. 1-55.

I. M. Gelfand and S. V. Fomin

1. Geodesic flows on manifolds of constant negative curvature, Uspehi Mat. Nauk vol. 7 (47) (1952) pp. 118-137.

A. GROTHENDIECK

1. Sur les espaces $(F)$ et $(D F)$, Summa Brasil. Math. vol. 3 (1954) pp. 57-121.

HARISH-ChANDRA

H. MAASS

1. Plancherel formula for the real $2 \times 2$ unimodular group, Proc. Nat. Acad. Sci. U.S.A. vol. 38 (1952) pp. 337-342.

1. Über eine neue Art von nichtanalytischen automorphen Funktionen und die Bestimmung Dirichletscher Reihen durch ihre Funktionalgleichungen, Math. Ann. vol. 121 (1949) pp. 141-183.

F. I. MAUTNER

1. Unitary representations of locally compact groups I, Ann. of Math. vol. 51 (1950) pp. 1-25.

2. Unitary representations of locally compact groups II, Ann. of Math. vol. 52 (1950) pp. 528-556.

3. Fourier analysis and symmetric spaces, Proc. Nat. Acad. Sci. U.S.A. vol. 37 (1951) pp. 529-533.

4. Note on the Fourier inversion formula on groups, Trans. Amer. Math. Soc. vol. 78 (1955) pp. 371-384.

5. Induced representations, Amer. J. Math. vol. 74 (1952) pp. 737-758.

6. Geodesic flows and unitary representations, Proc. Nat. Acad. Sci. U.S.A. vol. 40 (1954) pp. 33-36. 
S. Minakshisundaram and A. Pleijel

1. Eigenfunctions of the Laplace Operator on Riemann manifolds, Canad. J. Math. vol. 1 (1949) pp. 242-256.

R. E. A. C. Paley and N. Wiener

1. Fourier transform in the complex domain, Amer. Math. Soc. Colloquium Publications, vol. 19, 1934, 184 pp.

W. ROELCKE

1. Über die Wellengleichung bei Grenzkreisgruppen erster Art Sitzber, Heidelberg Ak. Wiss.,

L. SCHWARTZ Jahrg., 1953-1955, 4. Abh., pp. 159-267.

1. Etude des sommes d'exponentielles réelles, Hermann, Paris, 1943.

2. Approximation d'une fonction quelconque per des sommes d'exponentielles imaginaires, Ann. Fac. Sci. Univ. Toulouse vol. 6 (1942) pp. 111-174.

3. Thêorie generale des fonctions moyenne-periodique, Ann. of Math. vol. 48 (1947) pp. 857929.

4. Theorie des distributions, vols. I and II, Hermann, Paris, 1950-1951.

5. Analyse et synthese harmonique dans les espaces de distributions, Canad. J. Math. vol. 3 (1951) pp. 503-512.

6. Theorie des noyaux, Proceedings of the International Congress of Mathematicians, 1950, C. L. SIEGEL vol. 1, pp. 220-230.

1. Discontinuous groups, Ann. of Math. vol. 44 (1943) pp. 674-689.

E. C. Titchmarsh

1. The theory of functions, Oxford University Press, 1932.

2. Theory of Fourier integral, Oxford University Press, 1937.

3. The theory of the Riemann zeta function, Oxford University Press, 1951.

H. WHITNEY

1. On ideals of differentiable functions, Amer. J. Math. vol. 70 (1948) pp. 635-658.

INSTITUTE FOR Advanced Study,

Princeton, N. J.

JoHNS HopkINS UNIVERSITY,

BALTIMORE, MD. 